\title{
Splitting of the separatrices after a Hamiltonian-Hopf bifurcation under periodic forcing
}

\author{
E. Fontich, C. Simó, A. Vieiro \\ Departament de Matemàtiques i Informàtica \\ Universitat de Barcelona \\ BGSMath \\ Gran Via, 585, 08007, Barcelona, Spain
}

September 25, 2018

\begin{abstract}
We consider the effect of a non-autonomous periodic perturbation on a 2-dof autonomous system obtained as a truncation of the Hamiltonian-Hopf normal form. Our analysis focuses on the behaviour of the splitting of the invariant 2-dimensional stable/unstable manifolds. We analyse the different changes of dominant harmonic in the splitting functions. We describe how the dominant harmonics depend on the quotients of the continuous fraction expansion of the periodic forcing frequency. We have considered different frequencies including quadratic irrationals, frequencies having continuous fraction expansion with bounded quotients and frequencies with unbounded quotients. The methodology used is general enough to systematically deal with all these frequency types. All together allow us to get a detailed description of the asymptotic splitting behaviour for the concrete perturbation considered.
\end{abstract}

\section{Introduction}

In this work we consider the $\left(2+\frac{1}{2}\right)$-dof Hamiltonian system

$$
H\left(x_{1}, x_{2}, y_{1}, y_{2}, t\right)=H_{0}\left(x_{1}, x_{2}, y_{1}, y_{2}\right)+\epsilon H_{1}\left(x_{1}, x_{2}, y_{1}, y_{2}, t\right)
$$

where

$$
H_{0}\left(x_{1}, x_{2}, y_{1}, y_{2}\right)=x_{1} y_{2}-x_{2} y_{1}+\nu\left(\frac{x_{1}^{2}+x_{2}^{2}}{2}+\frac{y_{1}^{2}+y_{2}^{2}}{2}\left(-1+\frac{y_{1}^{2}+y_{2}^{2}}{2}\right)\right)
$$

and

$$
H_{1}\left(x_{1}, x_{2}, y_{1}, y_{2}, t\right)=\frac{y_{1}^{5}}{\left(d-y_{1}\right)(c-\cos (\theta))}, \quad \theta=\gamma t+\theta_{0}
$$

We shall fix concrete values of $c, d, \gamma$ and $\epsilon$, and consider $\nu>0$ as a perturbative parameter. The parameter $\theta_{0} \in[0,2 \pi)$ is an initial time phase.

Our motivation to consider that concrete system is to study some dynamical properties related to the Hamiltonian-Hopf bifurcation under a periodic forcing. Then, to start with, in Section 2 we briefly review the reduction to Sokolskii normal form (NF) for a 2-dof Hamiltonian system that undergoes a Hamiltonian-Hopf bifurcation. 
The truncation of the Sokolskii NF provides an integrable approximation of the dynamics. The above unperturbed system $H_{0}$ is simply the lowest order truncation that captures the main dynamical features of the Hamiltonian-Hopf bifurcation. Some basic facts concerning the dynamics of $H_{0}$ are summarized in Section 3 .

For $\nu>0$ the origin becomes of complex-saddle type. For $\epsilon=0$ the 2D stable/unstable invariant manifolds coincide. But for small and fixed $\epsilon>0$ the perturbation $H_{1}$ creates a splitting of these invariant manifolds. In Section 3 we also discuss some nice properties of the chosen perturbation. Such splitting of the invariant manifolds becomes exponentially small in $\nu$ as $\nu \rightarrow 0$. In Section 4 we perform a numerical computation of the splitting functions. Quadruple precision arithmetics is used to integrate (11) in order to get a sample of points on $W^{u}(0)$ and $W^{s}(0)$ that allows us to compute the splitting function in a fundamental domain. Different bifurcations are detected examining the nodal lines of the splitting functions as $\nu$ varies.

The corresponding Poincaré-Melnikov function is analytically investigated in Section 5 by means of a combination of numerical, symbolical and theoretical tools. The splitting problem considered is non-perturbative ( $\epsilon$ is fixed) and singular (when $\nu=0$ the system is not hyperbolic) and the use of the Melnikov approximation to study the splitting is not theoretically justified. In Section 6 we compare the results of the splitting obtained in Section 4 with those from (a suitable truncation, adding only the relevant terms) the Melnikov approximation derived in Section 5 , getting a remarkable agreement.

In Section 7 we further analyse the Poincaré-Melnikov function by taking advantage of the concrete properties of the system and of the perturbation to give explicit details of the asymptotic behaviour of the splitting. In particular, we look for the concrete values of the parameter $\nu$ for which a change in the dominant harmonic is detected and we study how these values asymptotically behave.

As expected, the Diophantine properties of the frequency $\gamma$ of the perturbation $H_{1}$ play a key role in the analysis performed in Section 7. Note that we do not assume that we have a concrete frequency, instead our hypothesis are on the properties of the continuous fraction expansion (CFE) of $\gamma$. Section 7.6 is devoted to illustrate the behaviour of the splitting for different frequencies $\gamma$. In particular, we show examples where some of the best approximants of $\gamma$ never become a dominant harmonic in the splitting functions.

Finally, Section 8 summarizes the results and describes related future work problems.

Five appendices complement the discussions through the text. In Appendix $\mathrm{A}$ we study the splitting under an autonomous perturbation of the unperturbed system. The simple asymptotic behaviour of the splitting is well-understood in this situation in contrast with the nonautonomous perturbation case studied in this work. Appendix B however illustrates that in the autonomous case, taking a non-entire perturbation, the analysis of the splitting by considering individual terms of the series expansion of the perturbation can lead to a larger dominant exponent of the Melnikov function. This is not expected in the non-autonomous case since the dominant term comes from the quasi-periodic properties of the splitting asymptotic behaviour. Appendix $\mathbb{C}$ discusses about the role of the regularity of the non-autonomous perturbation in $t$ in the asymptotic behaviour of the splitting.

When describing the asymptotic behaviour of the splitting of the invariant manifolds for system (11) we will see that for large intervals of $\nu$ the dominant harmonic coincides for both splitting functions. However, there are small intervals of $\nu$ where the dominant harmonics differ. In Appendix D we comment on the expected consequences that this fact has in what concerns the 
(local) diffusive properties of the system for very small values of $\nu$.

In the last appendix we focus on the presence of hidden harmonics, that is, harmonics associated to best approximants of $\gamma$ that never become a dominant harmonic of the splitting function. As said, hidden harmonics are shown for some frequencies in Section 7.6. We prove in Appendix E that, under generic conditions, it is not possible to have two consecutive best approximants of $\gamma$ which are not related to a dominant harmonic of the splitting function when some nearby quotients of the CFE of $\gamma$ are large enough. A more general situation can be found in [9].

The theoretical derivations presented in this work provide a satisfactory and complete description of the asymptotic behaviour of the splitting of separatrices of the system (1). On the other hand, a complete rigorous proof of the results included here will require

1. to bound the effect of higher order terms of the expansion of the splitting function in powers of $\epsilon$ to guarantee that the first order Poincaré-Melnikov function provides the dominant term of the splitting behaviour, and

2. to check that the contribution of the non-dominant harmonics of the Poincaré-Melnikov approximation does not change the dominant term of the asymptotic expansion of the splitting behaviour.

Even if we do not address formally any of the previous items, the numerical results that we present provide a strong numerical evidence supporting them.

\section{The theoretical framework: the Hamiltonian-Hopf bifurcation}

For the reader's convenience, in this section we briefly summarize some details of the analysis of the Hamiltonian-Hopf bifurcation.

Consider a one-parameter family of Hamiltonian systems $H_{\hat{\nu}}\left(x_{1}, x_{2}, y_{1}, y_{2}\right)$ which undergo a Hamiltonian-Hopf bifurcation. Assume that for $\hat{\nu}>0$ the origin is elliptic and becomes complex unstable for $\hat{\nu}<0$. This implies that the eigenvalues of the linearised Hamiltonian system suffer a Krein collision: for $\hat{\nu}>0$ the linear system has two pairs of purely imaginary eigenvalues $\pm \mathrm{i} \omega_{1}$ and $\pm \mathrm{i} \omega_{2}$. These pairs meet in a double pair $\pm \mathrm{i} \omega, \omega>0$, on the imaginary axis for $\hat{\nu}=0$ (Krein collision) and they become a hyperbolic quartet $\pm \alpha \pm \mathrm{i} \omega, \alpha, \omega>0$ for $\hat{\nu}<0$.

Let $\mathbb{P}_{k}$ be the set of homogeneous polynomials of degree $k \in \mathbb{N}$. Consider the Taylor expansion at 0 of $H_{\hat{\nu}}$ expressed as

$$
H_{\hat{\nu}}=\sum_{k \geq 2} \sum_{j \geq 0} \hat{\nu}^{j} H_{k, j}, \quad \text { where } H_{k, j} \in \mathbb{P}_{k} \text { for all } k \geq 2, j \geq 0 .
$$

The first step is to reduce the quadratic part $H_{2,0}$ to a canonical NF (i.e. a NF obtained via a symplectic change of coordinates). After doing this reduction the strategy will be to use a Lie series methodology to successively (order by order) simplify (as much as possible) the terms $H_{2, j}, j \geq 1$, and $H_{k, j}, k \geq 3, j \geq 0$.

The possible canonical forms for quadratic Hamiltonians were obtained in [28. In the case of two pairs of (double) purely imaginary eigenvalues $H_{2,0}$ can be reduced to the so-called Williamson $\mathrm{NF}$

$$
H_{2,0}=-\omega\left(x_{2} y_{1}-x_{1} y_{2}\right)+\frac{1}{2}\left(x_{1}^{2}+x_{2}^{2}\right)
$$


The next step involves normalising higher order terms of $H_{\hat{\nu}}$. The fact that the linearization at the Hamiltonian-Hopf bifurcation point is non-semisimple makes the NF reduction a little bit more involved, see [19, 7, 18, 13, 21. A standard procedure to deal with the terms of order $(k, j)$ is to look for a change of variables given by the time-1 map of a Hamiltonian $G \in \mathbb{P}_{k}$. In such a case the corresponding change transforms $H_{\hat{\nu}}$ into

$$
\tilde{H}_{\hat{\nu}}=\sum_{i \geq 0} \frac{1}{i !} \operatorname{ad}_{H_{\hat{\nu}}}^{i}(G)
$$

where $\operatorname{ad}_{F}(G)=\{F, G\}$ denotes the usual adjoint operator defined in terms of the Poisson bracket

$$
\{F, G\}=\left(\frac{\partial F}{\partial x_{1}} \frac{\partial G}{\partial y_{1}}-\frac{\partial F}{\partial y_{1}} \frac{\partial G}{\partial x_{1}}\right)+\left(\frac{\partial F}{\partial x_{2}} \frac{\partial G}{\partial y_{2}}-\frac{\partial F}{\partial y_{2}} \frac{\partial G}{\partial x_{2}}\right) .
$$

Collecting the terms of $\tilde{H}_{\hat{\nu}}$ of order $(k, j)$ in (3) we get $H_{k, j}+\operatorname{ad}_{H_{2}}(G)$, meaning that the change of coordinates allows us to remove the terms $H_{k, j}$ of $H_{\hat{\nu}}$ that belong to $\operatorname{Im~ad}_{H_{2}}(G)$. The Fredholm alternative implies that $\mathbb{P}_{k}=\operatorname{Im~ad}_{H_{2}} \oplus \operatorname{Ker~ad}_{H_{2}}^{\top}$, where $\operatorname{ad}_{H_{2}}^{\top}$ denotes the transpose operator. Then, as indicated in [8, 18], a systematic way to proceed is to look, at each order $(k, j)$ of the normalisation procedure, for $G \in \mathbb{P}_{k}$ such that

$$
H_{k, j}+\operatorname{ad}_{H_{2}}(G) \in \operatorname{Ker}_{H_{2}}^{\top} .
$$

Moreover, in the (symplectic, $\Omega=d x_{1} \wedge d y_{1}+d x_{2} \wedge d y_{2}=d R \wedge d r+d \Theta \wedge d \theta$ ) new coordinates

$$
y_{1}=r \cos (\theta), \quad y_{2}=r \sin (\theta), \quad R=\left(x_{1} y_{1}+x_{2} y_{2}\right) / r, \quad \Theta=x_{2} y_{1}-x_{1} y_{2},
$$

the transpose linear system (i.e. the system with equations defined by the matrix $J\left(D^{2} H_{2}\right)^{\top}$ ) reduces to $H_{2}^{\top}=-\omega \Theta+\frac{1}{2} r^{2}$, see [18]. Then (44) implies that the normalised (formal) Hamiltonian is given by

$$
\mathrm{NF}\left(H_{\hat{\nu}}\right)=\omega \Gamma_{1}+\Gamma_{2}+\sum_{\substack{k, l, j \geq 0 \\ k+l+j \geq 2}} a_{k, l, j} \Gamma_{1}^{k} \Gamma_{3}^{l} \hat{\nu}^{j},
$$

where

$$
\Gamma_{1}=x_{1} y_{2}-x_{2} y_{1}, \quad \Gamma_{2}=\left(x_{1}^{2}+x_{2}^{2}\right) / 2 \quad \text { and } \quad \Gamma_{3}=\left(y_{1}^{2}+y_{2}^{2}\right) / 2 .
$$

This is the so-called Sokolskii NF [27, see [18, 10] for further details on its derivation. Note that:

- We have seen that there exists a formal change of variables $C$ (not convergent in general) such that reduces the given system to the NF (6). Moreover, $C$ is symplectic, see for example [26]. If the quadratic part of the original system is already in Williamson NF, then the change is near-the-identity.

- The reduced Hamiltonian (6) is formally integrable and possesses $\Gamma_{1}$ as an extra (formal) integral of motion. The original Hamiltonian is only formally integrable (that is, the truncation at any order is integrable) and the difference between the Hamiltonian $H_{\hat{\nu}}$ and the formal series $\mathrm{NF}\left(H_{\hat{\nu}}\right) \circ C^{-1}$ is beyond all orders.

- The reduction to a NF is achieved by means of successive changes of coordinates to normalize order by order the full Hamiltonian. Each of the changes of the normalization procedure reduces the domain where the truncated NF gives a good approximation. For a fixed perturbation parameter $\hat{\nu}$, there is an optimal truncation order of the NF that minimizes the bound of the error between the Hamiltonian and the NF in a suitable domain around the fixed point. Note that the optimal order depends discontinuously on $\hat{\nu}$ because it jumps on the integers. See, e.g., [20, 25] 
Next we discuss some features of the invariant manifolds of the origin for $\mathrm{NF}\left(H_{\hat{\nu}}\right)$. In particular, $\left\{\Gamma_{1}, \Gamma_{2}\right\}=0$ and $\left\{\Gamma_{1}, \Gamma_{3}\right\}=0$ and hence, as we have said, $\Gamma_{1}$ is a first integral of $\operatorname{NF}\left(H_{\hat{\nu}}\right)$. Therefore $\Gamma_{1}=0$ on the invariant manifolds of the origin. On the other hand, these manifolds lie on $\operatorname{NF}\left(H_{\hat{\nu}}\right)=0$. From (6) , making explicit the lowest order terms of $\mathrm{NF}\left(H_{\hat{\nu}}\right)$, we have

$$
\begin{aligned}
\operatorname{NF}\left(H_{\hat{\nu}}\right)= & \omega \Gamma_{1}+\Gamma_{2}+\hat{\nu}\left(a_{1,0,1} \Gamma_{1}+a_{0,1,1} \Gamma_{3}\right)+a_{2,0,0} \Gamma_{1}^{2}+a_{1,1,0} \Gamma_{1} \Gamma_{3}+a_{0,2,0} \Gamma_{3}^{2} \\
& +\mathcal{O}\left(\hat{\nu}^{2}\left(\Gamma_{1}+\Gamma_{3}\right), \hat{\nu}\left(\Gamma_{1}+\Gamma_{3}\right)^{2},\left(\Gamma_{1}+\Gamma_{3}\right)^{3}\right) .
\end{aligned}
$$

Then, the $2 \mathrm{D}$ stable and unstable invariant manifolds $W^{s / u}(\mathbf{0})$ are given by the relation

$$
\Gamma_{2}+\hat{\nu} a_{0,1,1} \Gamma_{3}+a_{0,2,0} \Gamma_{3}^{2}+\mathcal{O}\left(\hat{\nu}^{2} \Gamma_{3}, \hat{\nu} \Gamma_{3}^{2}, \Gamma_{3}^{3}\right)=0
$$

We want to have real invariant manifolds $W^{s / u}(\mathbf{0})$, which requires $\Gamma_{2}, \Gamma_{3}>0$ (otherwise they lie in the complex domain). This means that $\hat{\nu} a_{0,1,1}<0$ and, since we have assumed that for $\hat{\nu}<0$ the origin is a complex-unstable fixed point, we must have $a_{0,1,1}>0$. Moreover, in such a case, for $a_{0,2,0}>0$ the invariant manifolds $W^{u / s}(0)$ live in a finite domain which, requiring the same order for the three dominant terms in (8), has size $\Gamma_{2}=\mathcal{O}\left(\hat{\nu}^{2}\right)$ and $\Gamma_{3}=\mathcal{O}(\hat{\nu})$. However for $a_{0,2,0}<0$ the invariant manifolds may be unbounded. For the first case we introduce the new parameter $\nu$ by $\hat{\nu}=-\nu^{2}$, and the rescaling $x_{i}=\nu^{2} \tilde{x}_{i}, \omega y_{i}=\nu \tilde{y}_{i}, i=1,2, \omega t=\tilde{t}$, see [19]. For concreteness, we shall consider $\nu>0$. After this non-canonical change of variables the system is again Hamiltonian and the corresponding Hamiltonian is

$$
\mathrm{NF}\left(\tilde{H}_{\nu}\right)=\tilde{\Gamma}_{1}+\nu\left(\tilde{\Gamma}_{2}+a \tilde{\Gamma}_{3}+\eta \tilde{\Gamma}_{3}^{2}\right)+\mathcal{O}\left(\nu^{2}\right)
$$

where

$$
a=-a_{0,1,1} / \omega^{2} \quad \text { and } \quad \eta=a_{0,2,0} / \omega^{4} .
$$

Hence, as it was pointed out in [17, for $\eta>0$ the invariant manifolds $W^{u / s}(0)$ are bounded while for $\eta<0$ they may be unbounded. Henceforth, we assume $a<0$ and $\eta>0$.

Remark 2.1. From (7) one checks that the eigenvalues of the linearisation at the origin of the original system $H_{\hat{\nu}}\left(x_{1}, x_{2}, y_{1}, y_{2}\right)$ are given by $\lambda= \pm \mathrm{i} \omega \pm \sqrt{a_{0,1,1}}(-\hat{\nu})^{1 / 2}+\mathcal{O}(\hat{\nu})$. Then, for $\hat{\nu}<0$, one has $\operatorname{Re}(\lambda)= \pm \omega \sqrt{-a}(-\hat{\nu})^{1 / 2}+\mathcal{O}(\hat{\nu})$ and $\operatorname{Im}(\lambda)= \pm \omega+\mathcal{O}(\hat{\nu})$.

\section{The system: a periodic perturbation of the truncated NF}

In this section we provide some details on the concrete system (1) studied in this paper.

\subsection{The unperturbed system}

Our starting point is the truncated (ignoring $\mathcal{O}\left(\nu^{2}\right)$ terms) Sokolskii NF Hamiltonian (9). According to Section 2, for $a<0$ and $\eta>0$ the invariant manifolds of the origin are bounded. The rescaling $\tilde{x}_{i} \rightarrow(-\sqrt{\eta} / a) \tilde{x}_{i}, \tilde{y}_{i} \rightarrow(\sqrt{-\eta / a}) \tilde{y}_{i}, i=1,2, \nu \rightarrow \sqrt{-a} \nu$, reduces the truncated Hamiltonian (9) to the case $a=-1$ and $\eta=1$. To simplify notation, we denote the rescaled variables and parameter simply by $\left(x_{1}, x_{2}, y_{1}, y_{2}\right)$ and $\nu$, respectively. We also introduce $\mathbf{x}=\left(x_{1}, x_{2}\right)$, $\mathbf{y}=\left(y_{1}, y_{2}\right)$, and we denote by $H_{0}$ the corresponding truncated Hamiltonian. Hence, $H_{0}$ is just given by

$$
H_{0}(\mathbf{x}, \mathbf{y})=\Gamma_{1}+\nu\left(\Gamma_{2}-\Gamma_{3}+\Gamma_{3}^{2}\right)
$$


where $\Gamma_{1}=x_{1} y_{2}-x_{2} y_{1}, \Gamma_{2}=\left(x_{1}^{2}+x_{2}^{2}\right) / 2$ and $\Gamma_{3}=\left(y_{1}^{2}+y_{2}^{2}\right) / 2$. The system $H_{0}$ is defined on the symplectic manifold $\left(\mathbb{R}^{4}, \Omega\right)$ with $\Omega=d x_{1} \wedge d y_{1}+d x_{2} \wedge d y_{2}$, and the equations of motion are

$$
\begin{array}{llrl}
\dot{x}_{1}=-x_{2}+\nu y_{1}\left(y_{1}^{2}+y_{2}^{2}-1\right), & \dot{y}_{1}=-y_{2}-\nu x_{1}, \\
\dot{x}_{2}=x_{1}+\nu y_{2}\left(y_{1}^{2}+y_{2}^{2}-1\right), & \dot{y}_{2}=y_{1}-\nu x_{2} .
\end{array}
$$

As follows from Section 2, $H_{0}$ is integrable and $\Gamma_{1}$ is an independent first integral of the system. The origin is a fixed point of (11) with eigenvalues $\pm \nu \pm \mathrm{i}$. For $\nu>0$, the origin is of complexsaddle type and the invariant manifolds $W^{u / s}(\mathbf{0})$ are given by $\left\{H_{0}=0\right\} \cap\left\{\Gamma_{1}=0\right\}$. To elucidate the dynamics of $H_{0}$ it is convenient to introduce (non-symplectic) polar coordinates

$$
x_{1}=R_{1} \cos \left(\psi_{1}\right), \quad x_{2}=R_{1} \sin \left(\psi_{1}\right), \quad y_{1}=R_{2} \cos \left(\psi_{2}\right), \quad y_{2}=R_{2} \sin \left(\psi_{2}\right),
$$

where $R_{1}, R_{2}>0$ and $\psi_{1}, \psi_{2} \in[0,2 \pi)$. The equations of motion become

$$
\begin{array}{ll}
\dot{R}_{1}=\nu R_{2}\left(R_{2}^{2}-1\right) \cos \left(\psi_{2}-\psi_{1}\right), & \dot{\psi}_{1}=1+\nu\left(R_{2}^{2}-1\right) R_{2} \sin \left(\psi_{2}-\psi_{1}\right) / R_{1}, \\
\dot{R}_{2}=-\nu R_{1} \cos \left(\psi_{2}-\psi_{1}\right), & \dot{\psi}_{2}=1+\nu R_{1} \sin \left(\psi_{2}-\psi_{1}\right) / R_{2} .
\end{array}
$$

One has $\Gamma_{1}=R_{1} R_{2} \sin \left(\psi_{2}-\psi_{1}\right)$, and hence $\Gamma_{1}=0$ implies $\sin \left(\psi_{2}-\psi_{1}\right)=0$. One can distinguish two cases: either $\psi_{1}-\psi_{2}=0(\bmod 2 \pi)$ or $\psi_{1}-\psi_{2}=\pi(\bmod 2 \pi)$. Each of these cases defines a system for $R_{1}, R_{2}>0$. But, since the changes $R_{1} \rightarrow-R_{1}$ and $R_{2} \rightarrow-R_{2}$ reduce the system of one of the cases to the other one, it is enough to consider one of the cases if one allows $R_{1}, R_{2}$ to be negative 1 To fix ideas, we consider $\psi_{1}-\psi_{2}=\pi(\bmod 2 \pi)$. Then, the restriction of the dynamics on $\left\{\Gamma_{1}=0\right\}$ for the $\left(R_{1}, R_{2}\right)$-components is just given by the equations related to the Duffing Hamiltonian $K=\nu\left(R_{1}^{2}-R_{2}^{2}+R_{2}^{4} / 2\right) / 2$ (with the symplectic 2 -form $\Omega_{K}=d R_{2} \wedge d R_{1}$ ). The local positive branch of the homoclinic orbit $\gamma(t)$ of $K$, with $R_{1}, R_{2}>0$, corresponds to the unstable manifold of the origin. It follows from (13) that along the invariant manifolds $\psi_{1}=\psi_{2}-\pi$ and $\psi_{2}=t+\psi_{0}$, where $\psi_{0} \in[0,2 \pi)$ is an arbitrary phase. Moreover, the invariant manifolds of the unperturbed system (11) are foliated by homoclinic orbits $\gamma_{\psi_{0}}(t)=\left(x_{1}(t), x_{2}(t), y_{1}(t), y_{2}(t)\right)$ given by

$$
x_{1}(t)=-R_{1}(t) \cos (\psi), \quad x_{2}(t)=-R_{1}(t) \sin (\psi), \quad y_{1}(t)=R_{2}(t) \cos (\psi), \quad y_{2}(t)=R_{2}(t) \sin (\psi),
$$

being $\psi=t+\psi_{0}, R_{1}(t)=\sqrt{2} \operatorname{sech}(\nu t) \tanh (\nu t)$, and $R_{2}(t)=\sqrt{2} \operatorname{sech}(\nu t)$. In particular, $\gamma_{\psi_{0}}(t)$ has singularities at $t=(2 n+1) \mathrm{i} \pi / 2 \nu, n \in \mathbb{Z}$.

\subsection{The perturbation}

We proceed by adding a periodic perturbation to (11). Concretely, as stated in the Introduction, we consider

$$
H(\mathbf{x}, \mathbf{y}, t)=H_{0}(\mathbf{x}, \mathbf{y})+\epsilon H_{1}(\mathbf{x}, \mathbf{y}, t)
$$

where $H_{0}(\mathbf{x}, \mathbf{y})$ is the unperturbed Hamiltonian (11), which depends on $\nu$, and

$$
H_{1}(\mathbf{x}, \mathbf{y}, t)=g\left(y_{1}\right) f(\theta)=\frac{y_{1}^{5}}{d-y_{1}} \frac{1}{c-\cos (\theta)},
$$

where $\theta=\gamma t+\theta_{0}$, with $\gamma \in \mathbb{R} \backslash \mathbb{Q}$ and $\theta_{0} \in[0,2 \pi)$ is an initial phase, $d>\sqrt{2}$ and $c>1$. The parameter $\epsilon$ is considered to be small and fixed. We choose $\gamma=(\sqrt{5}-1) / 2, d=7, c=5$

\footnotetext{
${ }^{1}$ The virtual singularities at $R_{1}=0$ and $R_{2}=0$ play no role. They are due to the use of polar coordinates. Indeed, using the so-called Sokolskii coordinates 27] one can remove one of them. We note, however, that there are not globally defined polar coordinates around a 2-dof complex-saddle singularity, see [15].
} 
and $\epsilon=10^{-3}$ for the majority of computations through the paper, but we do not restrict to these values in the theoretical considerations. In particular, we will give details on how to deal with other irrational frequencies $\gamma$ and the role of their arithmetic properties in the asymptotic splitting behaviour as $\nu \rightarrow 0$.

The following comments motivate and somehow justify the perturbation (15) considered.

1. A generic autonomous perturbation would create a splitting of separatrices. This case resembles the splitting of a $(1+1 / 2)$-dof Hamiltonian system (by considering the reduction to the energy level where the separatrices lie). A direct analysis of the Poincaré-Melnikov function in this case reveals an exponentially small behaviour in the parameter $\nu$ of the splitting measured as a variation of $\Gamma_{1}$. We summarize in Appendix $\mathrm{A}$ the theoretical results and some concrete numerical simulations of the behaviour of the splitting for an autonomous perturbation.

2. The phenomena becomes much richer under a non-autonomous perturbation since different frequencies interact. Consider the particular case of the perturbation (15). Around the invariant manifolds the unperturbed system possesses the internal frequency 1 in $t$, see (14). Then, we choose $\gamma \in \mathbb{R} \backslash \mathbb{Q}$ in $H_{1}$ so that the effect of the perturbation resembles that of a quasi-periodic forcing. Concretely, when one restricts the perturbation $\epsilon H_{1}(\mathbf{x}, \mathbf{y}, t)=\epsilon g\left(y_{1}\right) f(\theta)$ to the unperturbed invariant manifolds $W^{u / s}(\mathbf{0})$ of $H_{0}(\mathbf{x}, \mathbf{y})$, since $y_{1}$ has a factor periodic in $t$ as (14) shows, one gets a quasi-periodic function in $t$ with basic frequencies $(1, \gamma)$. As will be shown, some of the linear combinations of the basic frequencies are slower (hence they average in a worst way) and describe the behaviour of the dominant terms of the splitting of the invariant manifolds.

3. $H_{0}$ is an entire function of $\mathbf{x}, \mathbf{y}$. The perturbation $H_{1}$ in a neighbourhood of the unperturbed invariant manifolds is real analytic with respect to $\mathbf{x}, \mathbf{y}$ (because, in particular, $y_{1} \lesssim \sqrt{2}$ and we choose $d=7$ ) and it is analytic in $t$. This implies that the amplitude of the dominant term of the Poincaré-Melnikov approximation of the splitting function decreases exponentially in the parameter $\nu$, see details in Appendix C.

4. The Fourier coefficients of the even function $f(\theta)=(c-\cos (\theta))^{-1}=\sum_{j \geq 0} c_{j} \cos (j \theta)$ are given by

$$
c_{0}=1 / \sqrt{c^{2}-1}, \quad c_{j}=2 c_{0} /\left(c+\sqrt{c^{2}-1}\right)^{j} \quad \text { for } j \geq 1 .
$$

In particular, the Fourier coefficients decay as $1 /\left(c+\sqrt{c^{2}-1}\right)^{j}$ (this is related to the fact that $f(\theta)$ has poles at $\pm \mathrm{i} \log \left(c+\sqrt{c^{2}-1}\right)$ ).

On the other hand, the Taylor series of $g\left(y_{1}\right)$ is given by

$$
g\left(y_{1}\right)=\frac{y_{1}^{5}}{d-y_{1}}=\sum_{k \geq 0} d^{-k-1} y_{1}^{5+k} .
$$

It contains all powers $y_{1}^{k}$ for $k \geq 5$, but does not contain terms in the other three variables. The choice of $c=5$ and $d=7$ guarantees a fast enough decay but still allows us to differentiate the role of the different harmonics in the Poincaré-Melnikov function. See also Remark 3.2 below.

5. Finally, there is also a practical reason: $H_{1}$ is simple enough so that quadruple precision numerical integration of the full system can be carried out in a reasonable CPU time.

Remark 3.1. Note that the perturbation $H_{1}(\mathbf{x}, \mathbf{y}, t)$ preserves the fixed point at the origin. Instead one could consider perturbations such that the origin becomes a periodic orbit. We do not deal with this situation in this paper, but note that the description given here also applies to this case. 


\subsection{The splitting function}

In the following sections we study the invariant manifolds $W^{u / s}(\mathbf{0})$ of the system (1) and the asymptotic behaviour of their splitting as $\nu \rightarrow 0$. Here we introduce the notation we shall use to refer to the splitting function and its different approximations.

We write $H_{0}=G_{1}+\nu G_{2}$, where $G_{1}=\Gamma_{1}$ and $G_{2}=\Gamma_{2}-\Gamma_{3}+\Gamma_{3}^{2} . G_{1}$ and $G_{2}$ are first integrals of $H_{0}$. They are independent first integrals except for points on the surface $x_{1}=$ $\pm y_{2} \sqrt{y_{1}^{2}+y_{2}^{2}-1}, \quad x_{2}=\mp y_{1} \sqrt{y_{1}^{2}+y_{2}^{2}-1}$ (which includes, in particular, the origin and the periodic orbit $x_{1}=x_{2}=0, y_{1}^{2}+y_{2}^{2}=1$ ). The unperturbed invariant manifolds are given by $G_{1}=G_{2}=0$.

Given $\epsilon \geq 0$, for $i=1,2$, we denote by $F_{i}^{u}$ (resp. $F_{i}^{s}$ ) the restriction of $G_{i}$ to the invariant manifolds $W^{u}(\mathbf{0})$ (resp. $\left.W^{s}(\mathbf{0})\right)$. For $\epsilon$ small, the invariant manifolds $W^{u / s}(\mathbf{0})$ can be represented as graphs $g_{u / s}: \mathbb{R}^{2} \rightarrow \mathbb{R}^{4}, g_{u / s}\left(\psi_{0}, \theta_{0}\right)=\left(\psi_{0}, \theta_{0}, F_{1}^{u / s}\left(\psi_{0}, \theta_{0}\right), F_{2}^{u / s}\left(\psi_{0}, \theta_{0}\right)\right)$. Each component of the graph $g_{u / s}$ defines a 2-dimensional surface in $\mathbb{R}^{3}$, they are referred below by $F_{1}^{u / s}$-graph and $F_{2}^{u / s}$-graph of $W^{u}(\mathbf{0})$,

The splitting function $\left(\Delta F_{1}, \Delta F_{2}\right)$ is defined by

$$
\Delta F_{i}\left(\psi_{0}, \theta_{0}\right)=F_{i}^{u}\left(\psi_{0}, \theta_{0}\right)-F_{i}^{s}\left(\psi_{0}, \theta_{0}\right), \quad i=1,2
$$

The splitting function (18) can be expanded as

$$
\Delta F_{i}=\Delta F_{i}^{\{1\}}+\Delta F_{i}^{\{2\}}+\ldots,
$$

where $\Delta F_{i}^{\{k\}}\left(\psi_{0}, \theta_{0}\right)=\epsilon^{k} M_{k}\left(\psi_{0}, \theta_{0}\right),\left|M_{k}\right|=\mathcal{O}(1)$. Hence, $\left(\Delta F_{1}^{\{1\}}, \Delta F_{2}^{\{1\}}\right)$ is the first-order Poincaré-Melnikov approximation (in powers of $\epsilon$ ) of the splitting function.

Below, we perform direct numerical computations of the invariant manifolds to obtain approximations $\widetilde{F}_{i}^{u / s}$ of the components of the graph function, $i=1,2$. From them we compute numerical approximations $\widetilde{\Delta F_{i}}$ of the components of the splitting function $\Delta F_{i}$.

Finally, the first-order approximation $\left.\left(\Delta F_{1}^{\{1\}}, \Delta F_{2}^{\{1\}}\right)\right)$ can be expanded in Fourier series in $\left(\psi_{0}, \beta_{0}\right)$. Truncating them we obtain approximations that can be evaluated symbolically. In Section 6 we compare the results obtained symbolically from suitable truncations of $\left(\Delta F_{1}^{\{1\}}, \Delta F_{2}^{\{1\}}\right)$ with the numerical approximations $\left(\widetilde{\Delta F_{1}}, \widetilde{\Delta F_{2}}\right)$.

Remark 3.2. There are several theoretical works concerning the splitting of invariant manifolds in presence of a quasi-periodic forcing, we refer to [1, 26, 5, 3, 4, 2, A common hypothesis is that all the Fourier harmonics in $t$ and all the Taylor series terms in $\mathbf{x}, \mathbf{y}$ appear in the corresponding expansions. Then they use generic analytic decay of the coefficients to bound the dominant term of the Melnikov function. The perturbation considered in this work, although does not have all the required terms, behaves similarly. In future works we plan to investigate the effect of absence of harmonics and/or Taylor terms in the perturbation and the consequences it has in the behaviour of the splitting of the invariant manifolds and in the dynamics around them. In particular, a higher order Melnikov analysis could be needed to describe the splitting in such a situation. 


\section{Numerical computations of the splitting: dominant harmonics and nodal lines}

We present some numerical computations concerning the invariant manifolds $W^{u / s}(\mathbf{0})$ and their splitting for small values of $\nu$. We compute $\widetilde{\Delta F_{1}}$ for a mesh of points in a fundamental domain (see below) as the difference of the value $\tilde{F}_{1}^{u}$ obtained for a point on $W^{u}(\mathbf{0})$ and the "corresponding" point on $W^{s}(\mathbf{0})$. We describe below how to assign the corresponding point by using coordinates in a fundamental domain of the invariant manifolds. Similarly, we also compute $\widetilde{\Delta F_{2}}$.

It is useful to consider the Poincaré section $\Sigma=\max \left\{y_{1}^{2}+y_{2}^{2}\right\}=\max R_{2}^{2}$, see (12). Note that the invariant manifolds $W^{u / s}(\mathbf{0})$ of the unperturbed system $(\epsilon=0)$ intersect $\Sigma$ in the curve $x_{1}=x_{2}=0, y_{1}^{2}+y_{2}^{2}=2$. This is no longer true for $\epsilon>0$ because of the changes of order $\epsilon$ due to the perturbation (see Fig. 11). Moreover, there is a (exponentially small in $\nu$ ) splitting of the invariant manifolds $W^{u / s}(\mathbf{0})$ for $\epsilon \neq 0$.

The illustrations in this section are for $\gamma=(\sqrt{5}-1) / 2$ (golden frequency) and for values of $\nu$ of the form $\nu_{i}=2^{-i}, i \geq 0$. For the computation of the invariant manifolds and their splitting we proceed as follows:

1. We consider a fundamental domain of $W^{u}(\mathbf{0})$. This is given by a 2-dimensional torus $\mathcal{T}$.

2. The propagation of $\mathcal{T}$ up to $\Sigma$ gives a 2-dimensional torus, say $\mathcal{T}_{\Sigma}$. The invariant manifolds $W^{u / s}(\mathbf{0})$ in $\mathbb{R}^{4}$ are then given as the $\tilde{F}_{1}^{u / s}$ and the $\tilde{F}_{2}^{u / s}$-graphs over $\mathcal{T}_{\Sigma}$. The initial "angle" and "time" phases $\psi_{0}$ and $\theta_{0}$ are local coordinates in $\mathcal{T}_{\Sigma}$.

3. To get the $\widetilde{F}_{1}^{u / s}$ and $\widetilde{F}_{2}^{u / s}$-graphs over $\mathcal{T}_{\Sigma}$ we propagate a set $\left\{\tilde{\psi}_{0, k}, \tilde{\theta}_{0, j}\right\}, 0 \leq k, j \leq 512$, of initial points in $\mathcal{T}$ (i.e. a total number of $2^{18}$ initial conditions) until they reach the Poincaré section $\Sigma$. Concretely we select the initial conditions as follows. We fix $R_{2}=10^{-12}$, set $R_{1}=R_{2}\left(1-R_{2}^{2} / 2\right)$ and define $y_{1}^{u}=R_{2} \cos \left(\tilde{\psi}_{0}\right), y_{2}^{u}=R_{2} \sin \left(\tilde{\psi}_{0}\right), x_{1}^{u}=R_{1} \cos \left(\tilde{\psi}_{0}\right), x_{2}^{u}=$ $R_{1} \sin \left(\tilde{\psi}_{0}\right)$. This gives an initial condition on $W^{u}$. By symmetry, $y_{1}^{s}=y_{1}^{u}, y_{2}^{s}=y_{2}^{u}, x_{1}^{s}=$ $-x_{1}^{u}, x_{2}^{s}=-x_{2}^{u}$ defines an initial condition on $W^{s}$.

4. For the propagation step, the numerical integration is performed using an ad-hoc implemented high-order Taylor time-stepper scheme with quadruple precision.

5. To compute the difference (i.e. the splitting) between $W^{u}(\mathbf{0})$ and $W^{s}(\mathbf{0})$ we need to compare them at the same points of $\mathcal{T}_{\Sigma}$. Hence, we select an equispaced mesh of angles $\psi_{0}$ and $\theta_{0}$ within $\mathcal{T}_{\Sigma}$, and refine the initial conditions in $\mathcal{T}$ (we select the initial guess from the set of previously computed points in $\Sigma$ ) using a Newton method.

To give some illustrations we choose $\nu=2^{-4}$ and $\nu=2^{-6}$. For those two values of $\nu$ the $\widetilde{F}_{1}^{s}$-graph (resp. $\widetilde{F}_{2}^{s}$-graph) of the stable manifold $W^{s}(\mathbf{0})$ over $\mathcal{T}_{\Sigma}$ is shown in Fig. 1 left (resp. right).

There are no appreciable differences (using the scale of the plots) between the graphs corresponding to $W^{s}(\mathbf{0})$ shown in Fig. 1 and the corresponding plots for the graphs of the unstable manifold $W^{u}(\mathbf{0})$. This is because the splitting $\left(\Delta F_{1}, \Delta F_{2}\right)$ becomes exponentially small with respect to $\nu$. We show in Fig. 2 the splitting $\left(\widetilde{\Delta F_{1}}, \widetilde{\Delta F_{2}}\right)$ for the same values of $\nu$ as in Fig. 1 ,

We note that while the graphs remain similar for those selected values of $\nu$ (although the vertical range changes for the $\tilde{F}_{2}$-graph representations), see Fig. 1, the dominant harmonic of the Fourier 

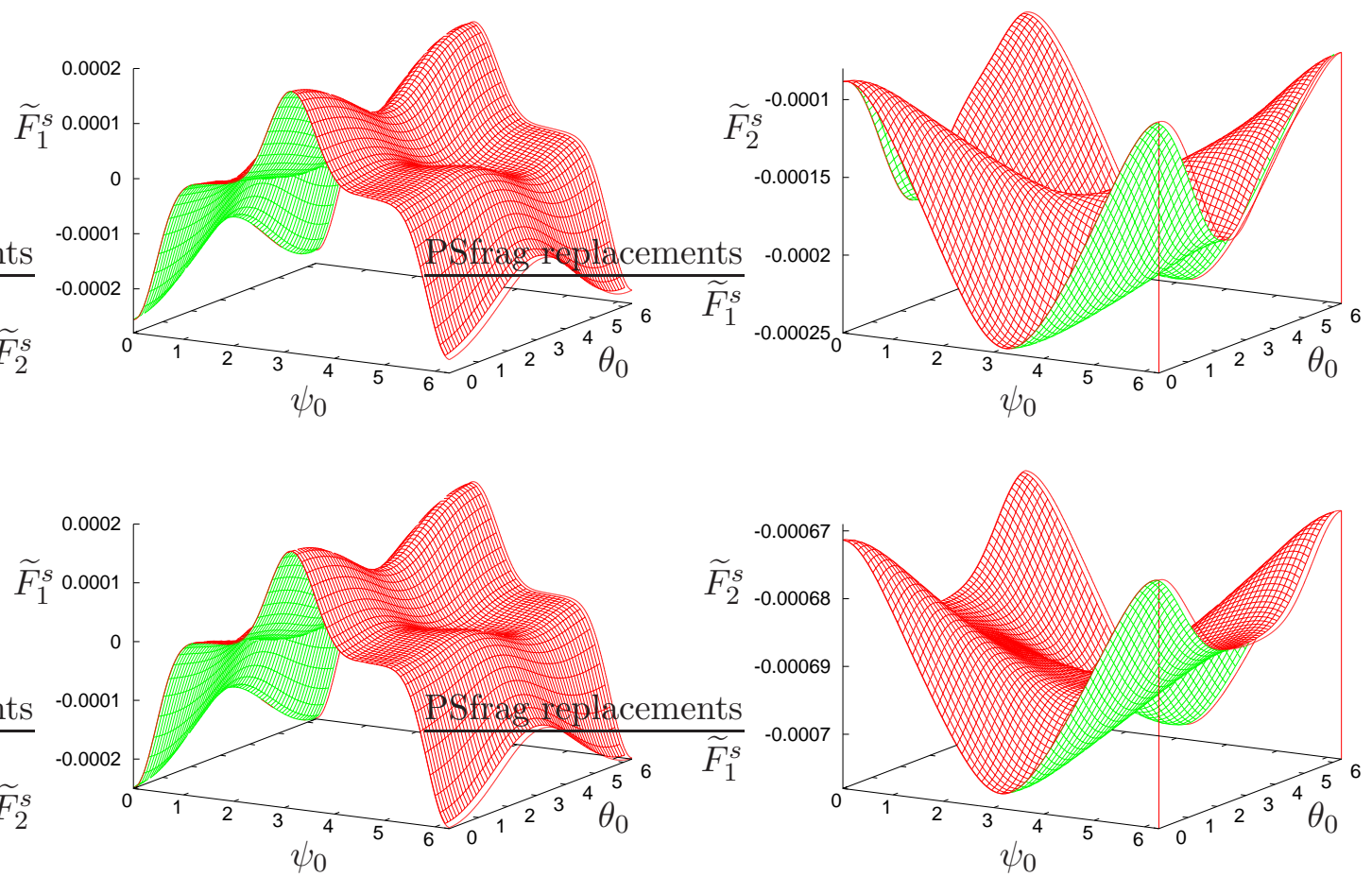

Figure 1: First row: for $\nu=2^{-4}$ we represent the $\widetilde{F}_{1}^{s}$-graph (left) and the $\widetilde{F}_{2}^{s}$-graph (right) of $W^{s}(\mathbf{0})$ over $\mathcal{T}_{\Sigma}$. Second row: the same for $\nu=2^{-6}$.
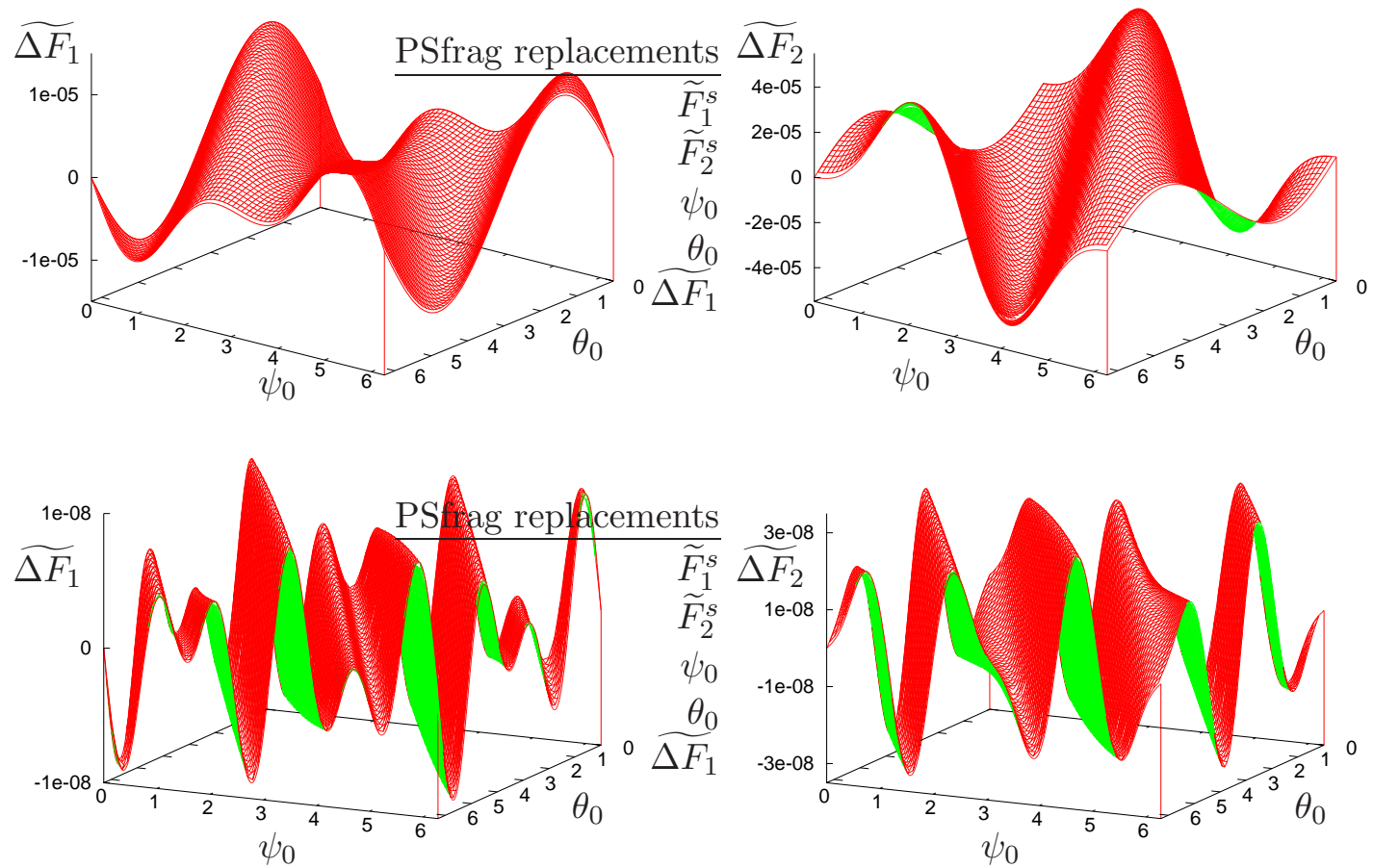

Figure 2: First row: For $\nu=2^{-4}$ we represent the $\widetilde{\Delta F_{1}}$ (left) and the $\widetilde{\Delta F_{2}}$ (right). Second row: $\widetilde{\Delta F_{1}}$ and $\widetilde{\Delta F_{2}}$ for $\nu=2^{-6}$. 


\begin{tabular}{|c|c|c|}
\hline$-\log _{2} \nu_{2}$ & $-\log _{2} \nu_{1}$ & Change of the dominant harmonics of $\widetilde{\Delta F_{1}}, \widetilde{\Delta F_{2}}$ \\
\hline 2.443 & 2.444 & $(1,0),(1,0) \longrightarrow(1,1),(1,0)$ \\
2.676 & 2.677 & $(1,1),(1,0) \longrightarrow(1,1),(1,1)$ \\
4.112 & 4.113 & $(1,1),(1,1) \longrightarrow(1,2),(1,1)$ \\
4.300 & 4.301 & $(1,2),(1,1) \longrightarrow(1,2),(1,2)$ \\
5.133 & 5.134 & $(1,2),(1,2) \longrightarrow(2,3),(1,2)$ \\
5.428 & 5.429 & $(2,3),(1,2) \longrightarrow(2,3),(2,3)$ \\
5.971 & 5.972 & $(2,3),(2,3) \longrightarrow(3,5),(2,3)$ \\
6.234 & 6.235 & $(2,3),(3,5) \longrightarrow(3,5),(3,5)$ \\
\hline
\end{tabular}

Table 1: The third column lists the dominant harmonic of $\widetilde{\Delta F_{1}}$ and the dominant harmonic of $\widehat{\Delta F_{2}}$ (both separated by a comma) for the value $\nu=\nu_{2}$ (left hand side of the arrow) and for $\nu=\nu_{1}$ (right hand side of the arrow). The $\left(m_{1}, m_{2}\right)$ harmonic corresponds to the frequency $m_{1} \psi_{0}-m_{2} \theta_{0}$ of the Fourier expansion of $\widetilde{\Delta F_{i}}$. The values of $\nu_{2}$ and $\nu_{1}$, shown in the first and second columns, are such that a bifurcation takes place for $\nu \in\left(\nu_{1}, \nu_{2}\right)$. We highlight the changes in the dominant harmonic of $\widetilde{\Delta F_{1}}$ in red while those of $\widetilde{\Delta F_{2}}$ are marked in blue.

expansion with respect to $\left(\psi_{0}, \theta_{0}\right) \in \mathcal{T}_{\Sigma}$ of $\widetilde{\Delta F_{1}}$ has changed from $\nu=2^{-4}$ to $\nu=2^{-6}$, see Fig. 2, A change of the dominant harmonic of $\widetilde{\Delta F_{2}}$ for these two values of $\nu$ is also observed. Moreover, for $\nu=2^{-6}$ the dominant harmonic of $\widetilde{\Delta F_{1}}$ is different from the dominant harmonic of $\widetilde{\Delta F_{2}}$, as can be appreciated from the number of oscillations of the left/right plots of the second row of Fig. 2. Concretely, for $\nu=2^{-4}$ the $(1,1)$ harmonic dominates for both $\widetilde{\Delta F_{1}}$ and $\widetilde{\Delta F_{2}}$, while for $\nu=2^{-6}$ the $(3,5)$-harmonic dominates for $\widetilde{\Delta F_{1}}$ and the $(2,3)$ harmonic dominates for $\widetilde{\Delta F_{2}}$.

We can look for the so-called nodal lines. These are the zero level curves of $\Delta F_{1}$ or $\Delta F_{2}$, i.e. where either the $F_{1}$-splitting or the $F_{2}$-splitting vanishes. For $\left(\psi_{0}, \theta_{0}\right) \in \mathcal{T}_{\Sigma}$ the nodal lines for some values of $2^{-4.301} \leq \nu \leq 2^{-2.443}$ are shown in Fig. 3. The nodal lines for some smaller values of $\nu$, up to $2^{-6.235}$, are shown in Fig. 4 . The values of $\nu$ shown have been selected so that a change of $10^{-3}$ in $\log _{2}(\nu)$ produces a topological change of the nodal lines. The intersections between the nodal lines correspond to homoclinic points and the changes in the topology of the nodal lines correspond to passages from a dominant harmonic to another one (either in $\Delta F_{1}$ or in $\Delta F_{2}$ ), see [26]. Hence, when decreasing $\nu$ many changes of dominant harmonic have been detected. We summarize them in Table 1. Concretely, we detect a topological change of the $\widetilde{\Delta F_{1}}$ or $\widetilde{\Delta F_{2}}$ nodal lines for $\nu \in\left(\nu_{1}, \nu_{2}\right)$. The values of $\nu_{1}$ and $\nu_{2}$ and the dominant harmonics at $\nu_{1}$ and $\nu_{2}$ are shown in the table.

As expected the dominant harmonics of $\widetilde{\Delta F_{1}}$ and $\widetilde{\Delta F_{2}}$ are the elements of the Fibonacci sequence, since they are related to the best approximants of the golden number frequency $\gamma$. Observe that the appearance of a new harmonic happens first for $\widehat{\Delta F_{1}}$ and later for $\widehat{\Delta F_{2}}$. These appearances take place alternatively. Later on we will estimate the changes in $\Delta F_{1}$ and $\Delta F_{2}$ carefully. The fact that the harmonics in $\Delta F_{1}$ and $\Delta F_{2}$ coincide for large ranges of $\nu$ has some dynamical consequences in the diffusion properties (see Appendix D).

\section{$5 \quad$ The splitting of the invariant manifolds}

For the unperturbed system $H_{0}$ given in (11) the 2-dimensional invariant manifolds of the origin $W^{u / s}(\mathbf{0})$ coincide. But this is no longer true for the perturbed system (15), the Hamiltonian 

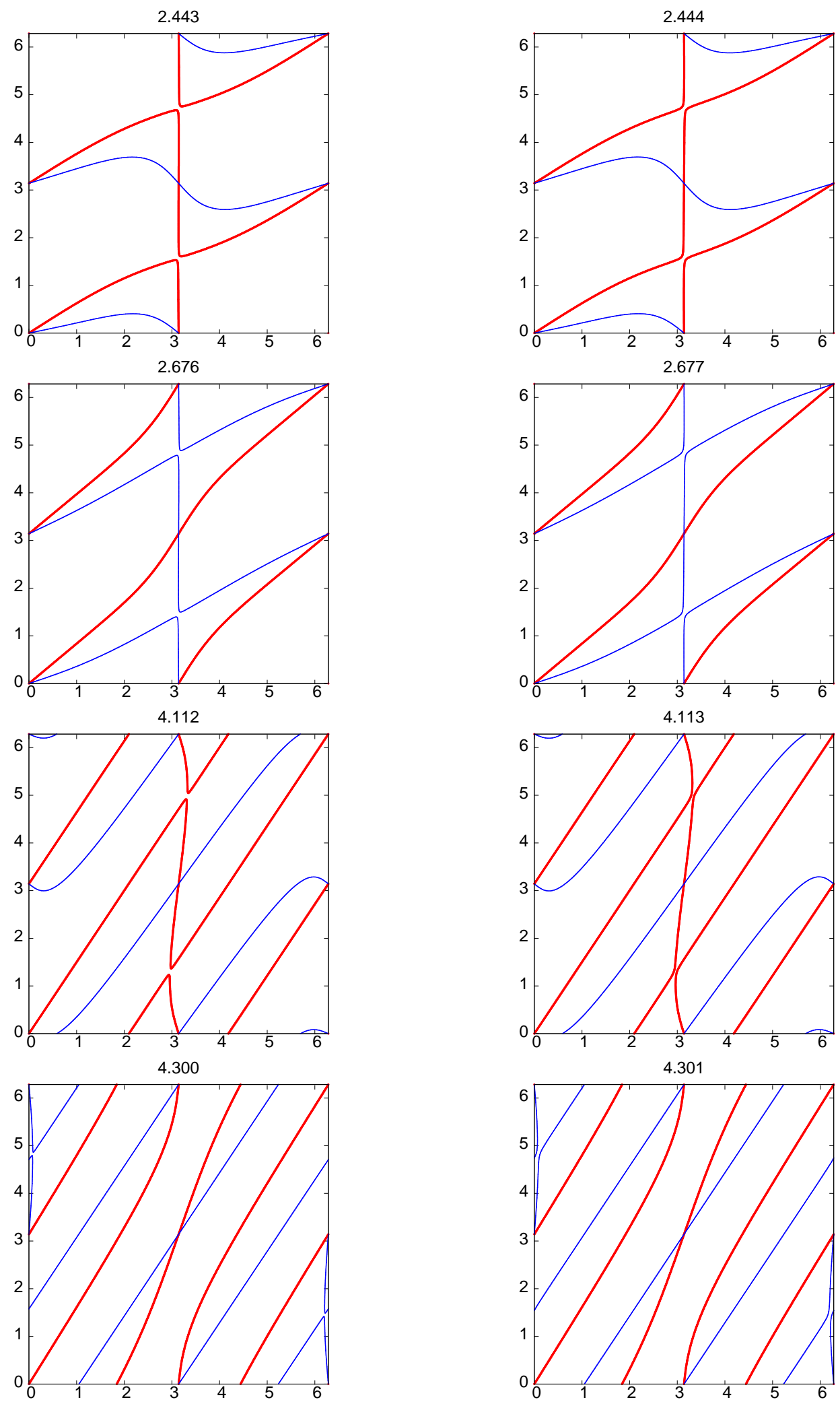

Figure 3: Nodal lines of $\widetilde{\Delta F_{1}}$ are shown in red. In blue we represent the ones related to $\widetilde{\Delta F_{2}}$. The squares $[0,2 \pi]^{2}$ represents the tori parameterized by $\left(\psi_{0}, \theta_{0}\right)$. Each row corresponds to two different values of the decreasing parameter $\nu$ : before (left) and after (right) the bifurcation (values of $\nu \geq 2^{-4.301}$ ). 

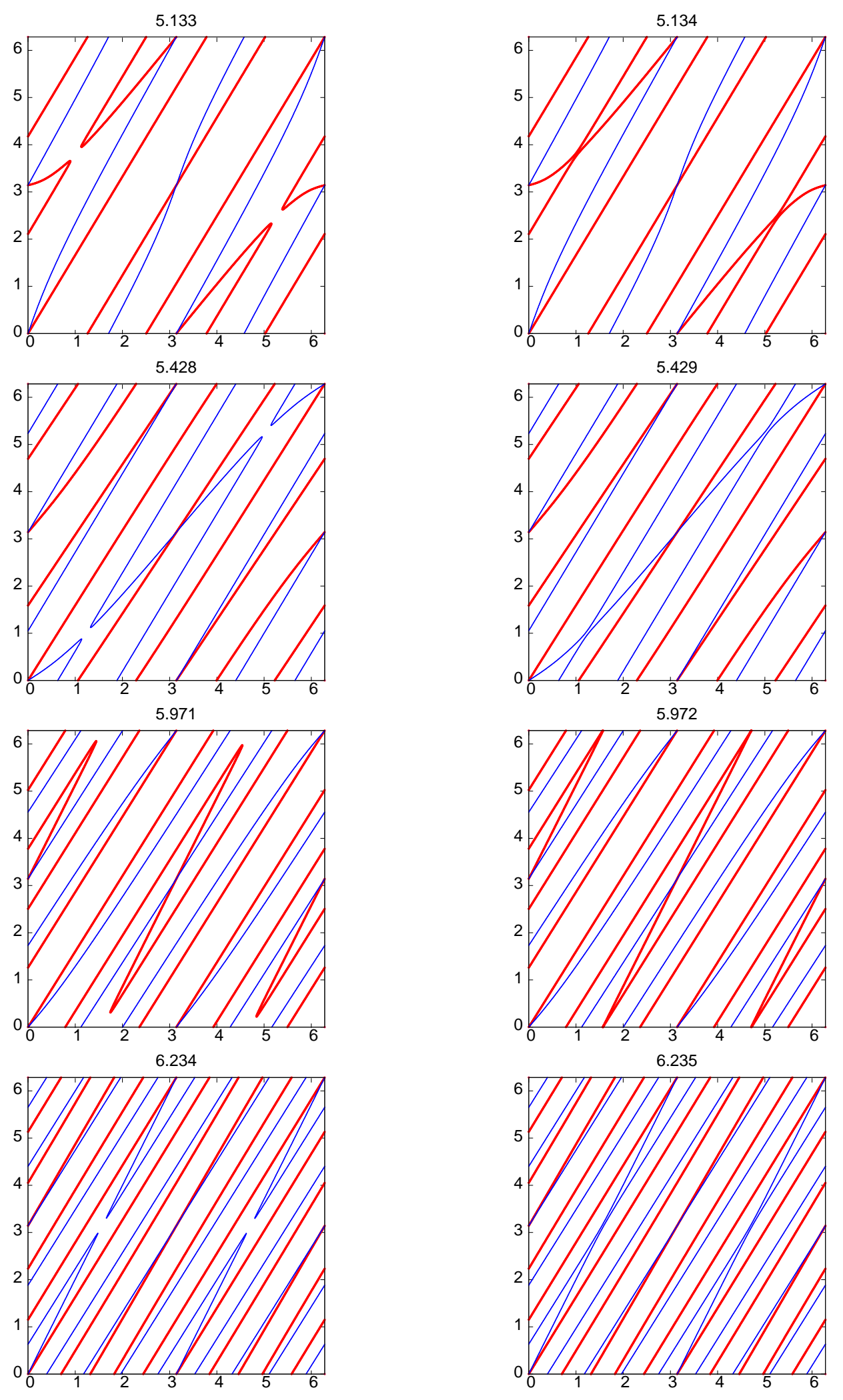

Figure 4: Continuation of Fig. 3: nodal lines for values of $2^{-6.235} \leq \nu<2^{-4.301}$. 
perturbation $\epsilon H_{1}(\mathbf{x}, \mathbf{y}, t)$ causes the splitting of the invariant manifolds $W^{u / s}(\mathbf{0})$. We will study the behaviour of the splitting of $W^{u / s}(\mathbf{0})$ as $\nu \rightarrow 0$ (i.e. as the system reduces hyperbolicity) for a fixed $\epsilon \neq 0$.

As it is well-known the splitting is related to the nearest singularities to the real axis of the time-parameterization of the unperturbed homoclinic trajectory. In our case the singularities are located at $\tau_{0}= \pm \mathrm{i} \pi / 2 \nu$. Moreover, the perturbation $H_{1}(\mathbf{x}, \mathbf{y})$ adds a space singularity $\rho$ located at $y_{1}=d$ and a time singularity related to $\hat{\theta}= \pm \mathrm{i} \log \left(c+\sqrt{c^{2}-1}\right)$ that restricts the domain of convergence of $f(\theta)$. The three singularities play a role in the asymptotic behaviour of the splitting as will be shown later on. We refer to [12] where a quasi-periodic perturbation with state singularities was considered.

\subsection{The derivation of the Poincaré-Melnikov function}

To obtain the expression for the Poincaré-Melnikov vector we proceed in a standard way so we just shortly describe its derivation.

Let $t_{0} \in \mathbb{R}, \zeta_{0}^{s}=\left(x_{0}^{s}, y_{0}^{s}\right) \in W^{s}(0), \zeta_{0}^{u}=\left(x_{0}^{u}, y_{0}^{u}\right) \in W^{u}(0)$ and $\zeta^{s, u}(t)=\left(x^{s, u}(t), y^{s, u}(t)\right)$ be the solutions of the Hamiltonian system $H_{0}+\epsilon H_{1}$ such that

$$
\zeta^{s, u}\left(t_{0}\right)=\zeta_{0}^{s, u}=\left(x_{0}^{s, u}, y_{0}^{s, u}\right)
$$

Clearly we have $\lim _{t \rightarrow \infty} \zeta^{s}(t)=\lim _{t \rightarrow-\infty} \zeta^{u}(t)=0$. Then, for $i=1,2$,

$$
\begin{aligned}
G_{i}\left(\zeta^{s}(t)\right) & -G_{i}\left(\zeta_{0}^{s}\right)=\int_{t_{0}}^{t} \frac{d}{d t}\left[G_{i} \circ \zeta^{s}\right](s) d s \\
& =\int_{t_{0}}^{t} D G_{i}\left(\zeta^{s}(s)\right)\left[J D H_{0}^{\top}\left(\zeta^{s}(s)\right)+\epsilon J D H_{1}^{\top}\left(\zeta^{s}(s)\right)\right] d s=\epsilon \int_{t_{0}}^{t}\left\{G_{i}, H_{1}\right\} \circ \zeta^{s}(s) d s
\end{aligned}
$$

and taking limit when $t$ goes to $\infty$ we get

$$
G_{i}\left(\zeta_{0}^{s}\right)=G_{i}\left(\zeta^{s}\left(t_{0}\right)\right)=-\epsilon \int_{t_{0}}^{\infty}\left\{G_{i}, H_{1}\right\} \circ \zeta^{s}(s) d s .
$$

In the same way

$$
G_{i}\left(\zeta_{0}^{u}\right)=G_{i}\left(\zeta^{u}\left(t_{0}\right)\right)=\epsilon \int_{t_{0}}^{-\infty}\left\{G_{i}, H_{1}\right\} \circ \zeta^{u}(s) d s .
$$

Actually $\zeta^{s, u}(t)$ depend on $\epsilon$. Let $\zeta^{0}(t)$ denote the solution of the system when $\epsilon=0$, with initial condition $\zeta_{0}$ for $t=t_{0}$. We use $\left(\psi_{0}, \theta_{0}\right)$, which parameterize the unperturbed manifold, to also parameterize $W^{s, u}(0)$, and we consider $\zeta_{0}^{s}, \zeta_{0}^{u}$ the points on $W^{s}(0), W^{u}(0)$ parameterized by $\left(\psi_{0}, \theta_{0}\right)$. Recall from Section 3.3 that $F_{i}^{u / s}$ denotes the restriction of $G_{i}$ to $W^{u / s}(\mathbf{0})$. By perturbation theory of invariant manifolds we have

$$
\begin{array}{ll}
\zeta^{s}(t)-\zeta^{0}(t)=O(\epsilon), & \text { uniformly in } t \text { for } t \in\left[t_{0}, \infty\right), \\
\zeta^{u}(t)-\zeta^{0}(t)=O(\epsilon), & \text { uniformly in } t \text { for } t \in\left(-\infty, t_{0}\right] .
\end{array}
$$

Since $\left(\psi_{0}, \theta_{0}\right)$ and $t_{0}$ are not independent we assume that $t_{0}=0$, that is, the corresponding Poincaré-Melnikov integrals depend on the two phase variables $\psi_{0}$ (initial "angle" phase, see (14)) and $\theta_{0}$ (initial "time" phase, see (15)). Therefore the splitting function is given by

$$
F_{i}^{u}\left(\zeta_{0}^{u}\right)-F_{i}^{s}\left(\zeta_{0}^{s}\right)=\epsilon \int_{-\infty}^{\infty}\left\{G_{i}, H_{1}\right\} \circ \zeta^{0}(s) d s+O\left(\epsilon^{2}\right)=: \epsilon M_{i}\left(\psi_{0}, \theta_{0}\right)+\mathcal{O}\left(\epsilon^{2}\right) .
$$

Below we denote by $\left(\Delta F_{1}^{\{1\}}\left(\psi_{0}, \theta_{0}\right), \Delta F_{2}^{\{1\}}\left(\psi_{0}, \theta_{0}\right)\right)=\left(\epsilon M_{1}\left(\psi_{0}, \theta_{0}\right), \epsilon M_{2}\left(\psi_{0}, \theta_{0}\right)\right)$ the so-called (first order) Poincaré-Melnikov approximation function. 


\subsection{The expression of the Poincaré-Melnikov integrals}

As before, see (15), we write $H_{1}(\mathbf{x}, \mathbf{y}, t)=g\left(y_{1}\right) f(\theta)$ where the expansions of $f$ and $g$ are given in (16) and (17), respectively. Since the Poisson brackets are

$$
\left\{G_{1}, H_{1}\right\}=y_{2} f(\theta) g^{\prime}\left(y_{1}\right), \quad\left\{G_{2}, H_{1}\right\}=x_{1} f(\theta) g^{\prime}\left(y_{1}\right),
$$

and

$$
g^{\prime}\left(y_{1}\right)=\sum_{k \geq 0} d_{k} y_{1}^{4+k}, \quad \text { where } d_{k}=(5+k) d^{-k-1},
$$

the Poincaré-Melnikov approximation of the splitting distance is

$$
\begin{aligned}
& \Delta F_{1}^{\{1\}}=4 \epsilon \int_{-\infty}^{\infty} \sin \left(t+\psi_{0}\right) f\left(\gamma t+\theta_{0}\right) \sum_{k \geq 0} \frac{\sqrt{2^{k+1}} d_{k}\left(\cos \left(t+\psi_{0}\right)\right)^{4+k}}{(\cosh (\nu t))^{5+k}} d t \\
& \Delta F_{2}^{\{1\}}=-4 \epsilon \int_{-\infty}^{\infty} f\left(\gamma t+\theta_{0}\right) \sum_{k \geq 0} \frac{\sqrt{2^{k+1}} d_{k}\left(\cos \left(t+\psi_{0}\right)\right)^{5+k} \sinh (\nu t)}{(\cosh (\nu t))^{6+k}} d t
\end{aligned}
$$

where, for simplicity, we have not written the dependence on $\psi_{0}, \theta_{0}$ in $\Delta F_{i}^{\{1\}}$.

Since the Poincaré-Melnikov integral is linear with respect to the perturbation $\epsilon H_{1}(\mathbf{x}, \mathbf{y}, t)$ we can write $M\left(\psi_{0}, \theta_{0}\right)=\left(M_{1}\left(\psi_{0}, \theta_{0}\right), M_{2}\left(\psi_{0}, \theta_{0}\right)\right)$ as an infinite sum and analyse the contribution to the splitting of each individual term of the series of $H_{1}$.

The Fourier series of the terms of the form $(\cos (\psi))^{m}$ and $(\cos (\psi))^{m} \sin (\psi)$, for $m \in \mathbb{Z}^{+}$, that appear in the previous equations are given by

$$
(\cos (\psi))^{m}=\sum_{i=0}^{E\left(\frac{m}{2}\right)} a_{m, i} \cos ((m-2 i) \psi), \quad(\cos (\psi))^{m} \sin (\psi)=\sum_{i=0}^{E\left(\frac{m+1}{2}\right)} b_{m, i} \sin ((m+1-2 i) \psi),
$$

where $E(x)$ denotes the integer part of $x$, and

$$
\begin{aligned}
a_{m, i} & =\frac{1}{2^{m-1}}\left(\begin{array}{c}
m \\
i
\end{array}\right), 0 \leq i<m / 2, \quad \text { and } \quad a_{m, m / 2}=\frac{1}{2^{m}}\left(\begin{array}{c}
m \\
m / 2
\end{array}\right) \text { if } m \text { even, } \\
b_{m, i} & =\frac{m+1-2 i}{2^{m}(m+1)}\left(\begin{array}{c}
m+1 \\
i
\end{array}\right), 0 \leq i \leq(m+1) / 2 .
\end{aligned}
$$

To compute the Poincaré-Melnikov integral for a general perturbation $g(\mathbf{x}, \mathbf{y}) f(\theta)$ the following comments apply:

- An expression of the form $x_{1}^{i_{1}} x_{2}^{i_{2}} y_{1}^{j_{1}} y_{2}^{j_{2}}$ in the Poisson bracket, when evaluated on the homoclinic orbit, becomes

$$
(-1)^{i_{1}+i_{2}} 2^{\left(i_{1}+i_{2}+j_{1}+j_{2}\right) / 2}(\cos (\psi))^{i_{1}+j_{1}}(\sin (\psi))^{i_{2}+j_{2}} \frac{(\sinh (\nu t))^{i_{1}+i_{2}}}{(\cosh (\nu t))^{2 i_{1}+2 i_{2}+j_{1}+j_{2}}} .
$$

The trigonometric terms can be reduced to the sum of expressions of the form $(\cos (\psi))^{m}$ or $(\cos (\psi))^{m} \sin (\psi)$, depending on whether $i_{2}+j_{2}$ is even or odd. In a similar way the hyperbolic terms can be reduced to the sum of negative powers of $\cosh (\nu t)$ or to such a sum times $\sinh (\nu t)$, depending on whether $i_{1}+i_{2}$ is even or odd. 
- Using the expansions (21)-(22), and assuming the expansion of the time-periodic part is

$$
f(\theta)=\sum_{j \geq 0} a_{j} \cos (j \theta)+\sum_{j>0} b_{j} \sin (j \theta), \quad a_{j}, b_{j} \in \mathbb{R}
$$

the integrals required to evaluate $\Delta F_{1}^{\{1\}}, \Delta F_{2}^{\{1\}}$ can be reduced to integrals of the product of $(\cosh (\nu t))^{-n}, n \geq 1$ or $(\cosh (\nu t))^{-n} \sinh (\nu t), n \geq 2$, by a function of the form

$$
\cos (k \psi) \cos (j \theta), \quad \cos (k \psi) \sin (j \theta), \quad \sin (k \psi) \cos (j \theta) \quad \text { or } \quad \sin (k \psi) \sin (j \theta), \quad k, j \in \mathbb{Z}^{+} .
$$

- Recall that $\psi=t+\psi_{0}$ and $\theta=\gamma t+\theta_{0}$. Expanding $f(\theta)$ and $\cos (\psi)$ and taking into account that the integrals of odd functions in $\mathbb{R}$ are zero, the computation of $\Delta F_{i}^{\{1\}}$, $i=1,2$, reduces to the computation of integrals of the form

$$
I_{1}(s, \nu, n)=\int_{\mathbb{R}} \frac{\cos (s t)}{(\cosh (\nu t))^{n}} d t, n \geq 1, \quad I_{2}(s, \nu, n)=\int_{\mathbb{R}} \frac{\sinh (\nu t) \sin (s t)}{(\cosh (\nu t))^{n}} d t, n \geq 2,
$$

for $\nu \neq 0$ (we will only be interested in $\nu>0$ ), where we have introduced the parameter $s=k \pm j \gamma$.

- Furthermore, one has

$$
I_{2}(s, \nu, n)=\frac{s}{\nu(n-1)} I_{1}(s, \nu, n-1), \quad n \geq 2 .
$$

Hence, it suffices to compute $I_{1}(s, \nu, n)$.

- One has

$$
I_{1}(s, \nu, 1)=\frac{\pi}{\nu} \frac{1}{\cosh (s \pi /(2 \nu))}, \quad I_{1}(s, \nu, 2)=\frac{s \pi}{\nu^{2}} \frac{1}{\sinh (s \pi /(2 \nu))},
$$

and, integrating by parts twice, one obtains

$$
I_{1}(s, \nu, n)=\frac{s^{2}+(n-2)^{2} \nu^{2}}{\nu^{2}(n-1)(n-2)} I_{1}(s, \nu, n-2), \quad n \geq 3 .
$$

That is,

$$
\nu^{n} I_{1}(s, \nu, n)=\frac{\pi}{(n-1) ! \cosh (s \pi /(2 \nu))} P_{n-1}(s, \nu), \quad \text { for } n \text { odd }
$$

and

$$
\nu^{n} I_{1}(s, \nu, n)=\frac{\pi}{(n-1) ! \sinh (s \pi /(2 \nu))} P_{n-1}(s, \nu), \quad \text { for } n \text { even, }
$$

where $P_{j}(s, \nu), j \geq 0$, are the homogeneous polynomials of degree $j$ in $(s, \nu)$ that satisfy the recurrence

$$
P_{0}(s, \nu)=1, \quad P_{1}(s, \nu)=s, \quad P_{j}(s, \nu)=\left(s^{2}+(j-1)^{2} \nu^{2}\right) P_{j-2}(s, \nu), j \geq 2 .
$$

In particular, we see that the terms in the series of $\Delta F_{i}^{\{1\}}, i=1,2$, decay to zero at least as $\exp (-|s| \pi /(2 \nu))$ as $\nu \rightarrow 0$. We note that, however, the functions $\Delta F_{i}^{\{1\}}, i=1,2$, may decay in a slower way, see Appendix B.

At this point we have all the ingredients to produce an algorithm to obtain expressions for $\Delta F_{1}^{\{1\}}, \Delta F_{2}^{\{1\}}$ with any accuracy. 
Remark 5.1. The analyticity domain in the spatial coordinates $(\mathbf{x}, \mathbf{y})$ and the analyticity strip in time $t$ of the perturbation $\epsilon H_{1}(\mathbf{x}, \mathbf{y}, t)$ can be, in general, of different size. Denote by $m(\mathbf{x}, \mathbf{y}, \theta)$ a term of the Taylor-Fourier expansion of $H_{1}(\mathbf{x}, \mathbf{y}, t)$, where $t=\left(\theta-\theta_{0}\right) / \gamma$. That is, $m(\mathbf{x}, \mathbf{y}, \theta)$ is a monomial of degree $k_{1} \geq 0$ in $(\mathbf{x}, \mathbf{y})$ with the harmonic $k_{2} \in \mathbb{Z}$ in $\theta$. Assume that there exist $\rho_{1}, \rho_{2}>0$ such that the coefficient $m$ of this monomial satisfies

$$
|m| \leq M \exp \left(-k_{1} \rho_{1}-\left|k_{2}\right| \rho_{2}\right)
$$

with $M>0$ and where $(\mathbf{x}, \mathbf{y})$ belongs to a compact domain containing the unperturbed real separatrices 2 Then, the contribution $T\left(k_{1}, k_{2}\right)$ of the monomial to the Poincaré-Melnikov integral is of the form

$$
T\left(k_{1}, k_{2}\right) \sim \epsilon A \nu^{B} \exp \left(-k_{1} \rho_{1}-\left|k_{2}\right| \rho_{2}\right) \exp \left(\frac{-|s| \pi}{2 \nu}\right), \text { with } s=k_{1}-\left|k_{2}\right| \gamma, A>0 .
$$

We note that it may happen that $T\left(k_{1}, k_{2}\right)$ dominates the behaviour of the splitting for $\nu$ small even if $k_{1}$ and $k_{2}$ (and the total order $k=k_{1}+\left|k_{2}\right|$ ) are large provided that $k_{1}-\left|k_{2}\right| \gamma$ is small enough. For example, consider $\rho:=\rho_{1}=\rho_{2}$ and assume that $\gamma$ verifies $\left|k_{1}-\right| k_{2}|\gamma|>C|k|^{-\tau}$ with $\tau \geq 1$. Then $T\left(k_{1}, k_{2}\right) \sim T(k)=\epsilon A \nu^{B} \exp (-k \rho) \exp \left(-C \pi / 2 \nu k^{\tau}\right)$ and the largest contribution is obtained for $k=k_{*} \sim(C \pi \tau / 2 \rho \nu)^{1 /(1+\tau)}$, which gives a term $T\left(k_{*}\right)=\mathcal{O}\left(\exp \left(-c / \nu^{1 /(\tau+1)}\right)\right)$. This agrees, provided $\tau>1$, with the exponentially small remainder obtained after an optimal number of steps of the averaging procedure for a quasi-periodic function, see details in [25]. When $\tau=1$ there are many terms that give the same contribution and the exponentially small (in $\nu$ ) upper bound in the averaging procedure gains an extra logarithmic term [23].

Summarizing, using (21) and (22), we can rewrite (201) as

$$
\begin{aligned}
& \Delta F_{1}^{\{1\}}=\epsilon \int_{-\infty}^{\infty} \sum_{k \geq 0} \sum_{0 \leq 2 i \leq 4+k} \sum_{j \geq 0} d_{k} b_{4+k, i} c_{j} 2^{\frac{5+k}{2}} \frac{1}{(\cosh (\nu t))^{5+k}} \sin ((k+5-2 i) \psi) \cos (j \theta) d t, \\
& \Delta F_{2}^{\{1\}}=-\epsilon \int_{-\infty}^{\infty} \sum_{k \geq 0} \sum_{0 \leq 2 i \leq 5+k} \sum_{j \geq 0} d_{k} a_{5+k, i} c_{j} 2^{\frac{5+k}{2}} \frac{\sinh (\nu t)}{(\cosh (\nu t))^{6+k}} \cos ((k+5-2 i) \psi) \cos (j \theta) d t .
\end{aligned}
$$

Taking into account that $\psi=t+\psi_{0}, \theta=\gamma t+\theta_{0}$, and expanding the terms $\sin \left(\ell\left(t+\psi_{0}\right)\right) \cos (j(\gamma t+$ $\left.\left.\theta_{0}\right)\right)$ and $\cos \left(\ell\left(t+\psi_{0}\right)\right) \cos \left(j\left(\gamma t+\theta_{0}\right)\right)$, where $\ell=k+5-2 i$, in the previous expression one reduces to evaluate integrals $I_{1}(s, \nu, n)$ and $I_{2}(s, \nu, n)$, given by (23), where $s=\ell \pm j \gamma$. Concretely, using the expansions

$$
\begin{aligned}
\sin \left(\ell\left(t+\psi_{0}\right)\right) & \cos \left(j\left(\gamma t+\theta_{0}\right)\right)=\frac{1}{2}\left[\sin \left((\ell+j \gamma) t+\ell \psi_{0}+j \theta_{0}\right)+\sin \left((\ell-j \gamma) t+\ell \psi_{0}-j \theta_{0}\right)\right] \\
= & \frac{1}{2}\left[\sin ((\ell+j \gamma) t) \cos \left(\ell \psi_{0}+j \theta_{0}\right)+\cos ((\ell+j \gamma) t) \sin \left(\ell \psi_{0}+j \theta_{0}\right)\right] \\
& +\frac{1}{2}\left[\sin ((\ell-j \gamma) t) \cos \left(\ell \psi_{0}-j \theta_{0}\right)+\cos ((\ell-j \gamma) t) \sin \left(\ell \psi_{0}-j \theta_{0}\right)\right], \\
\cos \left(\ell\left(t+\psi_{0}\right)\right) & \cos \left(j\left(\gamma t+\theta_{0}\right)\right)=\frac{1}{2}\left[\cos \left((\ell+j \gamma) t+\ell \psi_{0}+j \theta_{0}\right)+\cos \left((\ell-j \gamma) t+\ell \psi_{0}-j \theta_{0}\right)\right] \\
= & \frac{1}{2}\left[\cos ((\ell+j \gamma) t) \cos \left(\ell \psi_{0}+j \theta_{0}\right)-\sin ((\ell+j \gamma) t) \sin \left(\ell \psi_{0}+j \theta_{0}\right)\right] \\
& +\frac{1}{2}\left[\cos ((\ell-j \gamma) t) \cos \left(\ell \psi_{0}-j \theta_{0}\right)-\sin ((\ell-j \gamma) t) \sin \left(\ell \psi_{0}-j \theta_{0}\right)\right],
\end{aligned}
$$

\footnotetext{
${ }^{2}$ In particular, this assumption holds for the concrete example (15) considered in this paper for $c>1$ and $d>\sqrt{2}$, see the expansions (16) and (17).
} 
one obtains

$$
\begin{aligned}
& \Delta F_{1}^{\{1\}}=\epsilon \sum_{j \geq 0} c_{j} \sum_{k \geq 0} 2^{\frac{3+k}{2}} d_{k} \sum_{0 \leq 2 i \leq 4+k} b_{4+k, i} \sum_{l= \pm 1} I_{1}(k+5-2 i+l j \gamma, \nu, k+5) \sin \left((k+5-2 i) \psi_{0}+l j \theta_{0}\right), \\
& \Delta F_{2}^{\{1\}}=-\epsilon \sum_{j \geq 0} c_{j} \sum_{k \geq 0} 2^{\frac{3+k}{2}} d_{k} \sum_{0 \leq 2 i \leq 5+k} a_{5+k, i} \sum_{l= \pm 1} I_{2}(k+5-2 i+l j \gamma, \nu, k+6) \sin \left((k+5-2 i) \psi_{0}+l j \theta_{0}\right) .
\end{aligned}
$$

\section{Comparison between the splitting and the Melnikov approx- imation}

Note that our example fits within a non-perturbative ( $\epsilon$ is considered fixed) singular (when $\nu \rightarrow 0$ the system loses hyperbolicity) splitting case as described in [6]. The fact that the splitting is well-approximated by the Poincaré-Melnikov (vector) approximation $\epsilon M\left(\psi_{0}, \theta_{0}\right)$ must be justified in this context since, a priori, the $\mathcal{O}\left(\epsilon^{2}\right)$ error terms in the Poincaré-Melnikov approach can dominate for small enough values of $\nu$.

That is, in order to justify the use of the Poincaré-Melnikov approach one has to estimate the relative error term by checking that the constant in $\mathcal{O}\left(\epsilon^{2}\right)$ decays together with $\nu$ in an exponentially small way, becoming dominated by the (exponentially small in $\nu$ ) term $\mathcal{O}(\epsilon)$. The necessity of estimating the relative error was observed in [24], see also [6, 11].

The rigorous justification of the validity of the Melnikov integral is an interesting but difficult problem. In this work we are not going to deal with it. Instead, in this subsection, we choose $\gamma=(\sqrt{5}-1) / 2$ and we compare the amplitude of the splitting $\widetilde{\Delta F_{1}}$ and $\widetilde{\Delta F_{2}}$ computed directly using numerical methods (i.e. computing the invariant manifolds and the difference between them in a mesh of points) as explained in Section 4 with the values obtained by using the Poincaré-Melnikov integral and the recurrences detailed in Section 5.2 .

Let us clarify what we refer to by amplitude of the splitting. When we proceed numerically we compute the maximum of the absolute value of the distance between the invariant stable/unstable manifolds attained in a fundamental domain (i.e. on a torus parameterized by the angles $\left.\left(\psi_{0}, \theta_{0}\right)\right)$. On the other hand, when we proceed by evaluating the first order PoincaréMelnikov approximation using the expressions (25), we only take into account those terms that, for the value of $\nu$ considered, give a relative contribution larger than $10^{-10}$ to the total sum. Adding the contributions of these harmonic terms we obtain an approximation of $\Delta F_{i}^{\{1\}}$. In the following, both quantities are referred as amplitude of the splitting and are denoted by $\left|\Delta F_{i}^{\{1\}}\right|$.

For $\gamma=(\sqrt{5}-1) / 2$ we have computed, for more than 1000 values of $\log _{2}(\nu)$, the amplitude of the splitting using both approaches. The results are displayed in Fig. 5. Note that from Remark 5.1, since we have taken a constant type frequency $\gamma$, we expect the contribution of each term of the Taylor-Fourier expansion of the splitting function to be $\mathcal{O}(\exp (-c / \sqrt{\nu}))$. Accordingly we display $\sqrt{\nu} \log \left(\left|\Delta F_{i}^{\{1\}}\right| / \epsilon\right)$ as a function of $\log _{2}(\nu)$ in the figure. The direct numerical computations are done up to $\nu \lesssim 2^{-7}$, since for smaller values of $\nu$ they require a large number of digits and a large computing time. As an example, for $\epsilon=10^{-3}, \nu=2^{-10}$ one has $\left|\Delta F_{i}^{\{1\}}\right|=\mathcal{O}\left(10^{-32}\right)$. However, we can compute the Poincaré-Melnikov integral up to much smaller values of $\nu$.

Note the excellent agreement between the numerical and the theoretical methodologies. This accurate agreement supports the fact that the first order Melnikov integral asymptotically de- 


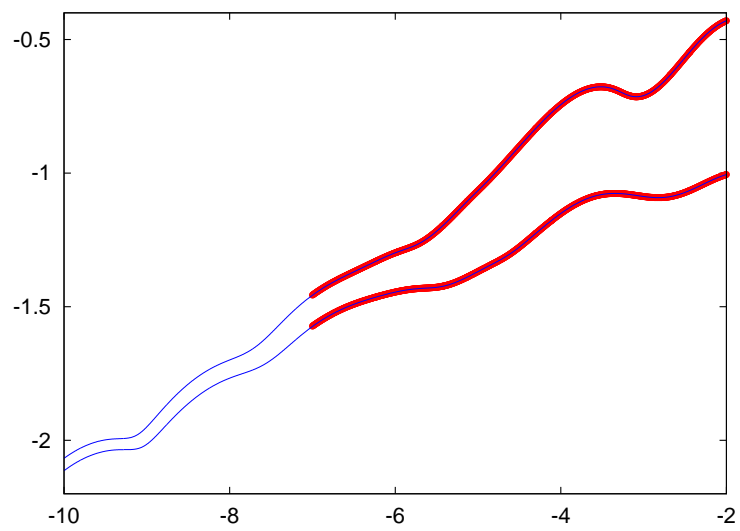

Figure 5: We represent $\sqrt{\nu} \log \left(\left|\Delta F_{i}^{\{1\}}\right| / \epsilon\right)$, for $i=1$ (bottom curve) and $i=2$ (top curve), as a function of $\log _{2}(\nu)$. In red points we show the direct numerical computations of the amplitude of the splitting. The blue line shows the values obtained using the Poincaré-Melnikov integrals through the expressions derived theoretically to evaluate them.

scribes the splitting. In particular, this numerical check makes us confident to investigate the asymptotic behaviour of the splitting for smaller values of $\nu$ using the first order approximation of the splitting function given by the Poincaré-Melnikov integral. This is the goal of the next section.

Finally, in Fig. [6 left we display the values of $\Delta F_{i}^{\{1\}}, i=1,2$, for different $\nu \in\left[2^{-24}, 2^{-2}\right]$. We have used a grid with spacing 0.005 in $\log _{2}(\nu)$. In the right plot we show the number of harmonics that contribute to $\Delta F_{i}^{\{1\}}, i=1,2$. Each harmonic comes from the contribution of different $(i, j, k)$-terms in expression (25), where, as before, we only have taken into account those terms with relative contribution larger than $10^{-10}$. For the largest values of $\nu$ considered in the figure the dominant harmonic is computed as a combination of up to 14 different terms. The values of $\Delta F_{i}^{\{1\}}, i=1,2$, are shown in the left panel of the same figure. We observe, in particular, that for $\nu<2^{-12}$ the number of harmonics used to compute the splitting function reduces to one with the exception of small intervals of $\nu$ where two terms are used. This is related to the dominant harmonics of the splitting function: for most values of $\nu$ only one term is relevant in $\Delta F_{i}^{\{1\}}$ meaning that there is a dominant harmonic of the splitting function, but when a change of dominant harmonic of the splitting function takes place one has to consider two terms (meaning that two harmonics have a similar contribution in such a range of $\nu$ ). Note also that the length of the interval of $\nu$ where the computation of $\Delta F_{i}^{\{1\}}$ requires two harmonics decreases as $\nu \rightarrow 0$.

\section{Asymptotic properties of the splitting behaviour}

\subsection{The theoretical results}

Here we state the main theoretical results which are proven in the following subsections.

Consider system (15) for $0 \leq \nu<\bar{\nu} \ll 1$ small enough, with $\epsilon>0, c>1, d>\sqrt{2}$ and $\gamma \in \mathbb{R} \backslash \mathbb{Q}$. 

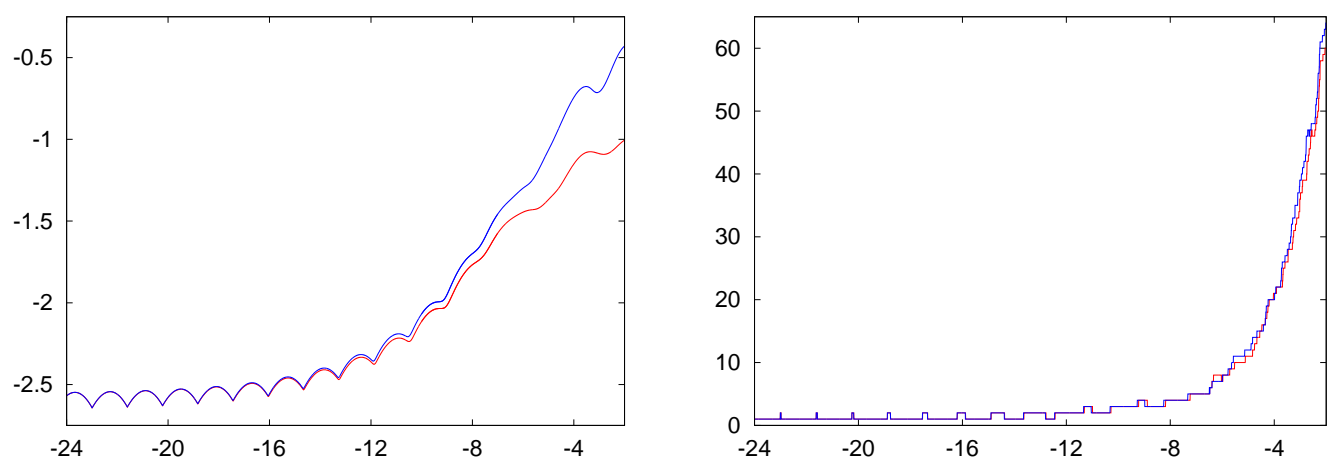

Figure 6: Left: We represent $\sqrt{\nu} \log \left(\left|\Delta F_{i}^{\{1\}}\right| / \epsilon\right), i=1,2$. Right: Number of harmonic terms considered to compute $\Delta F_{i}^{\{1\}}$. In both panels $\log _{2}(\nu)$ ranges in the horizontal axis.

From (25), we express $\Delta F_{i}^{\{1\}}$ as

$$
\Delta F_{i}^{\{1\}}=\epsilon \sum_{m_{1} \geq 0} \sum_{m_{2} \in \mathbb{Z}} \hat{C}_{m_{1}, m_{2}}^{(i)} \sin \left(m_{1} \psi_{0}-m_{2} \theta_{0}\right), \quad i=1,2,
$$

where $\hat{C}_{m_{1}, m_{2}}^{(i)} \in \mathbb{R}$. We introduce the notation $C_{m_{1}, m_{2}}^{(i)}=\left|\hat{C}_{m_{1}, m_{2}}^{(i)}\right|$ to denote the amplitudes of the Fourier modes of $M_{i}\left(\psi_{0}, \theta_{0}\right)=\Delta F_{i}^{\{1\}}\left(\psi_{0}, \theta_{0}\right) / \epsilon$.

Given $m_{1} / m_{2}, m_{j} \in \mathbb{Z} \backslash\{0\}, j=1,2$, an approximant of $\gamma$, let $c_{s, m_{1} / m_{2}}>0$ be the constant such that

$$
|s|=\left|m_{1}-\gamma m_{2}\right|=\frac{1}{c_{s, m_{1}, m_{2}} m_{1}} .
$$

The constants $c_{s, m_{1} / m_{2}}$ are related to the arithmetic properties of $\gamma$ (see Section 7.6). We shall denote the constant $c_{s, m_{1} / m_{2}}$ by $c_{s, n}$ when $m_{1} / m_{2}$ is a best approximant of $\gamma$ (in the sense of the continuous fraction expansions (CFE) of $\gamma$ ) and, in this case, $n$ refers to the order of the best approximant.

The following result provides a quantitative description of the way the different harmonics contribute to $\Delta F_{i}^{\{1\}}$.

Theorem 7.1. There exists a universal function $\Psi_{1}(L)$ such that

$$
\Psi_{1}(L)_{\mid L=c_{s, m_{1} / m_{2}} \nu m_{1}^{2}} \approx \sqrt{c_{s, m_{1} / m_{2}} \nu} \log C_{m_{1}, m_{2}}^{(1)},
$$

asymptotically when $\nu \rightarrow 0$. The function $\Psi_{1}(L)$ only depends on $\gamma$ through the additive term $k / \gamma$, where $k=c+\sqrt{c^{2}-1}$. On the other hand, the function

$$
\Psi_{2}(L)=\Psi_{1}(L)-\frac{\sqrt{L} \log L}{m_{1}}
$$

satisfies

$$
\Psi_{2}(L)_{\mid L=c_{s, m_{1} / m_{2}} \nu m_{1}^{2}} \approx \sqrt{c_{s, m_{1} / m_{2}} \nu} \log C_{m_{1}, m_{2}}^{(2)},
$$

asymptotically when $\nu \rightarrow 0$.

The proof of Theorem 7.1 is given in Sections 7.2 and 7.3 . 
If, from the arithmetic properties of $\gamma$, one can determine the asymptotic behaviour of the constants $c_{s, m_{1} / m_{2}}$, assuming that they have some defined asymptotic behaviour, then one can determine the dominant term (or dominant terms) of the splitting. When $\nu \rightarrow 0$ the dominant terms of the splitting are related to best approximants of $\gamma$. Our numerical results and theoretical discussions support the following conjecture.

Conjecture 7.1. Let $\left(\nu_{0}, \nu_{1}\right), \nu_{0}, \nu_{1}<\bar{\nu} \ll 1$, be an interval such that for all $\nu \in\left(\nu_{0}, \nu_{1}\right)$ the dominant harmonic in $\Delta F_{i}^{\{1\}}$ is the one associated to the best approximant $m_{1} / m_{2}$ of $\gamma$. Then, for $\nu \in\left(\nu_{0}, \nu_{1}\right)$,

$$
\left|\Delta F_{i}^{\{1\}}\right| \approx \epsilon \exp \left(\frac{\Psi_{i}(L)}{\sqrt{c_{s, m_{1} / m_{2} \nu}}}\right), \quad L=c_{s, m_{1} / m_{2}} \nu m_{1}^{2}, \quad i=1,2,
$$

where $\Psi_{2}(L)=\Psi_{1}(L)+\mathcal{O}\left(\sqrt{c_{s, m_{1} / m_{2}}}\right), \Psi_{1}(L)=\Psi_{M}+O\left(\left|L-L_{M}\right|^{2}\right)$, being $\Psi_{M}=\Psi_{1}\left(L_{M}\right)=$ $\max \left(\Psi_{1}(L)\right) \approx-4.860298$ and $L_{M} \approx 0.26236$.

Remark 7.1. Notice that $c_{s, m_{1} / m_{2}}$ depends on $\nu$ through the arithmetic properties of $\gamma$, as was explained in Remark 5.1. When $\gamma$ is a quadratic irrational then the constants $c_{s, m_{1} / m_{2}}$ remain bounded as $\nu \rightarrow 0$. On the other hand, when the quotients of the CFE of $\gamma$ are unbounded then the maxima of the constants $c_{s, m_{1} / m_{2}}$ grow when $\nu \rightarrow 0$. Actually the exponents in the exponentially small part of $\left|\Delta F_{i}^{\{1\}}\right|, i=1,2$, depend on $\nu$ through the behaviour of $\nu c_{s, m_{1} / m_{2}}$. See Section 7.6 for some examples.

Explicit expressions of the functions $\Psi_{i}(L)$ are derived in the following subsections. Note that $\Psi_{1}$ does not depend on the arithmetic properties of $\gamma$ and $\Psi_{2}$ depends only on $m_{1}$. The dependence is through $L$ which depends on the constant $c_{s, m_{1} / m_{2}}$ and the approximant $m_{1} / m_{2}$. This allows us to provide a methodology to study the asymptotic behaviour for any frequency $\gamma$. Note that the dominant harmonic $\left(m_{1}, m_{2}\right)$ changes when $\nu \rightarrow 0$ and so does the constant $c_{s, m_{1} / m_{2}}$. This allows us to study the values of $\nu$ for which a change of the dominant harmonic in $\Delta F_{i}^{\{1\}}$ is expected.

Conjecture 7.1 asserts that the dominant term of the series expansion of the Melnikov function gives the correct exponent of the splitting behaviour, that is, that

$$
\sqrt{\nu} \log \left(\frac{\left|\Delta F_{i}^{\{1\}}\right|}{\epsilon}\right) \approx \sqrt{\nu} \log C_{m_{1}, m_{2}}^{(i)} \approx \frac{1}{\sqrt{c_{s, m_{1} / m_{2}}}} \Psi_{i}(L), \quad L=c_{s, m_{1} / m_{2}} \nu m_{1}^{2},
$$

where the second approximation comes from Theorem 7.1. See Section 7.5 for a more detailed discussion. Here we just want to emphasize that this dominant term, which is related to the approximants of $\gamma$, has a larger order of magnitude than the remaining terms of the series. For example, for a constant type $\gamma$, if the dominant harmonic corresponds to the linear combination $s=m_{1}-m_{2} \gamma$, then one expects $m_{1}, m_{2} \sim 1 / \sqrt{\nu}$ (see Remark 5.11). This gives a term of order $\exp (-c / \sqrt{\nu})$ much larger than the order $\exp (-c / \nu)$ expected for the terms with other combinations. We stress that this is a purely quasi-periodic effect related to the existence of two frequencies in the system. Indeed, if one considers a one frequency forcing of the 1-dimensional separatrix dynamics, the effect of any of the terms of the Melnikov series can be of the same (or similar) order than the dominant one. Hence all terms can contribute to change the dominant exponent, see Appendix B]

\subsection{The amplitude of the harmonics associated to approximants of $\gamma$}

We consider first $M_{1}\left(\psi_{0}, \theta_{0}\right)=\Delta F_{1}^{\{1\}} / \epsilon$. Given $m_{1}, m_{2} \in \mathbb{Z}$ we look for the expression of $C_{m_{1}, m_{2}}^{(1)}=\left|\hat{C}_{m_{1}, m_{2}}^{(1)}\right|$ in (26). In (25) we choose $l=-1$ to get the more relevant terms, then one 
has $k=m_{1}+2 i-5$ and $j=m_{2}$ so that

$$
\begin{aligned}
C_{m_{1}, m_{2}}^{(1)} & =c_{m_{2}} \sum_{i \geq 0} 2^{\left(m_{1}+2 i-2\right) / 2} d_{m_{1}+2 i-5} b_{m_{1}+2 i-1, i} I_{1}(s, \nu, k+5) \\
& \approx c_{m_{2}} \sum_{i \geq 0} 2^{\left(m_{1}+2 i-2\right) / 2} d_{m_{1}+2 i-5} b_{m_{1}+2 i-1, i} \frac{2 \pi}{\nu} \frac{(s / \nu)^{m_{1}+2 i-1}}{\left(m_{1}+2 i-1\right) !} \Pi_{m_{1}+2 i}(\nu / s) \exp \left(-\frac{\pi s}{2 \nu}\right),
\end{aligned}
$$

where $s=\left|m_{1}-m_{2} \gamma\right|$ and $\Pi_{r}(\nu / s)=s^{1-r} P_{r-1}(s, \nu), r \geq 1$. Note that to lighten the notation we have used $s$ for $|s|$ in this section, hoping that no confusion will be produced.

From (24) one has the recurrence

$$
\Pi_{1}=\Pi_{2}=1, \quad \Pi_{r}(w)=\left(1+(r-2)^{2} w^{2}\right) \Pi_{r-2}(w), r \geq 2,
$$

where $w=\nu / s$. In the expression for $C_{m_{1}, m_{2}}^{(1)}$ above (and the one for $C_{m_{1}, m_{2}}^{(2)}$ at the end of this section) we have used the approximations $\cosh ^{-1}(\pi s /(2 \nu)), \sinh ^{-1}(\pi s /(2 \nu)) \sim 2 \exp (-\pi s /(2 \nu))$, valid when $w^{-1}=s / \nu$ is large enough, the relative error being $\mathcal{O}(\exp (-\pi s / \nu))$.

From (16), (19) and (21) it follows that

$$
c_{m_{2}}=\frac{2}{\rho_{c}^{m_{2}} \sqrt{c^{2}-1}}, d_{m_{1}+2 i-5}=\frac{m_{1}+2 i}{d^{m_{1}+2 i-4}}, \quad b_{m_{1}+2 i-1, i}=\frac{m_{1}}{2^{m_{1}+2 i-1}\left(m_{1}+2 i\right)}\left(\begin{array}{c}
m_{1}+2 i \\
i
\end{array}\right),
$$

where $\rho_{c}=c+\sqrt{c^{2}-1}$. Then,

$$
C_{m_{1}, m_{2}}^{(1)} \approx=\frac{2}{\rho_{c}^{m_{2}} \sqrt{c^{2}-1}} \frac{2 \pi}{\nu} d^{3} \exp \left(-\frac{\pi s}{2 \nu}\right) m_{1} 2^{-m_{1} / 2}\left(\frac{s}{\nu d}\right)^{m_{1}-1} \frac{d}{m_{1} !} S_{A},
$$

where $S_{A}$ denotes the sum

$$
S_{A}=\sum_{i \geq 0} A_{i}, \quad A_{i}=A_{i}\left(m_{1}, \nu, s\right)=\frac{m_{1}+2 i}{2^{i}\left(m_{1}+i\right) ! i !}\left(\frac{s}{\nu d}\right)^{2 i} \frac{m_{1} !}{d} \Pi_{m_{1}+2 i}(\nu / s) .
$$

Similarly, for $\Delta F_{2}^{\{1\}}$ one obtains, given $m_{1}, m_{2}$, that

$$
C_{m_{1}, m_{2}}^{(2)} \approx \frac{2}{\rho_{c}^{m_{2}} \sqrt{c^{2}-1}} \frac{2 \pi s}{\nu^{2}} d^{3} \exp \left(-\frac{\pi s}{2 \nu}\right) 2^{-m_{1} / 2}\left(\frac{s}{\nu d}\right)^{m_{1}-1} \frac{d}{m_{1} !} S_{A} .
$$

According to (28)-(30) the contribution of the integral to $\Delta F_{i}^{\{1\}}, i=1,2$, related to the approximant $m_{1} / m_{2}$ is $\mathcal{O}\left(\exp \left(-\frac{\pi s}{2 \nu}\right)\right)$, where the contribution of finite negative powers of $\nu$ has been neglected. Hence those harmonics associated to the smallest values of $s$ play the most important role. These are expected to be related (asymptotically as $\nu \rightarrow 0$ ) with the best approximants of $\gamma$.

From the expressions of $C_{m_{1}, m_{2}}^{(i)}, i=1,2$, we have the following result.

Proposition 7.1. Given $m_{1} \geq 0$ and $m_{2} \in \mathbb{Z}$ we have

$$
C_{m_{1}, m_{2}}^{(1)}=L C_{m_{1}, m_{2}}^{(2)}+\mathcal{O}(\exp (-\pi s / \nu))
$$

where $L=\nu m_{1}^{2} c_{s, m_{1} / m_{2}}$ and $c_{s, m_{1} / m_{2}}>0$ is such that $s=\left|m_{1}-\gamma m_{2}\right|=\frac{1}{c_{s, m_{1} / m_{2}} m_{1}}$. 
Proof. From (28) and (30) one has $C_{m_{1}, m_{2}}^{(1)} / C_{m_{1}, m_{2}}^{(2)} \approx m_{1} \nu / s=m_{1}^{2} c_{s, m_{1} / m_{2}} \nu=L$ for all $m_{1} \geq 0$ and $m_{2} \in \mathbb{Z}$.

This relation explains the difference between $\Delta F_{1}$ and $\Delta F_{2}$ in Fig. 5 , see also Fig. 2 first row. Recall that it was numerically observed that the dominant harmonic of both splittings coincide

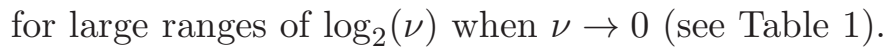

\subsection{The universal function $\Psi_{1}(L)$ associated to an approximant $m_{1} / m_{2}$ of $\gamma$.}

In this section we consider $m_{1} / m_{2} \approx \gamma$ an approximant (not necessarily a best approximant of $\gamma$ ). For concreteness we will focus on $\Delta F_{1}^{\{1\}}$, the expression of $\Psi_{2}(L)$ will follow directly from Proposition 7.1

Given $m_{1} / m_{2} \approx \gamma$, we express $C_{m_{1}, m_{2}}^{(1)}$ given by (28) as

$$
C_{m_{1}, m_{2}}^{(1)}=\mathcal{P}_{f} \mathcal{P}_{F} S_{A}, \quad \text { and } S_{A}=\mathcal{P}_{M} \mathcal{P}_{Q},
$$

where

$$
\mathcal{P}_{f}=\frac{4 \pi d^{4} m_{1}}{s \sqrt{c^{2}-1}}, \quad \mathcal{P}_{F}=\frac{1}{\rho_{c}^{m_{2}} 2^{m_{1} / 2} m_{1} !} \exp \left(-\frac{\pi s}{2 \nu}\right)\left(\frac{s}{\nu d}\right)^{m_{1}},
$$

and $\mathcal{P}_{M}$ denotes the dominant term of $S_{A}$ (i.e. the term of $S_{A}$ which gives the maximum contribution to the sum) and $\mathcal{P}_{Q}=S_{A} / \mathcal{P}_{M}$.

To get intuition about how to proceed we perform some numerical investigations considering (temporary!) $\gamma=(\sqrt{5}-1) / 2$. Fig. (7left shows the behaviour of $\Delta F_{1}^{\{1\}}$. We see different changes of dominant harmonic as $\nu \rightarrow 0$ that are marked with points. We represent the behaviour of $S_{A}=\mathcal{P}_{M} \mathcal{P}_{Q}$ for $\gamma=(\sqrt{5}-1) / 2$ in Fig. 7 right. These two terms play the role of a factor which ranges in a finite interval away from zero. In particular, this means that the change of harmonic should be detected in the prefactor $\mathcal{P}_{f} \mathcal{P}_{F}$. The important term is $\mathcal{P}_{F}$ since $\mathcal{P}_{f}$ does not depend on $\nu$ explicitly and, for the $m_{1} / m_{2}$ approximant giving the maximum contribution to the splitting function for a fixed $\nu$, behaves as a power of $\nu$, hence negligible in front the exponentially small term in $\nu$ of $\mathcal{P}_{F}$. Hence, below, we first look for the changes using just $\mathcal{P}_{F}$, later we will discuss the contribution of the sum $S_{A}$. The factor $\mathcal{P}_{F}$ depends on $m_{1}, m_{2}$ and $\nu$. We shall check later that $\mathcal{P}_{Q}$ gives no relevant contribution to $C_{m_{1}, m_{2}}^{(1)}$.

In what follows, given $m_{1} / m_{2} \approx \gamma$, we study the contribution of the different factors to $C_{m_{1}, m_{2}}^{(1)}$.

The contribution of $\mathcal{P}_{F}$. We write $\mathcal{P}_{F}=\mathcal{P}_{F}\left(m_{1}\right)$ to explicitly note its dependence on $m_{1}$. Using Stirling's formula we approximate $\log m_{1} ! \approx m_{1}\left(\log m_{1}-1\right)$ (i.e. we ignore the term $\sqrt{2 \pi m_{1}}$ ), one has

$$
\begin{aligned}
\log \left(\mathcal{P}_{F}\left(m_{1}\right)\right) & \approx-m_{1}\left(\frac{\log \left(\rho_{c}\right)}{\gamma}+\frac{\log 2}{2}+\left(\log m_{1}-1\right)+\frac{\pi}{2 c_{s, m_{1} / m_{2}} \nu m_{1}^{2}}+\log \left(d c_{s, m_{1} / m_{2}} \nu m_{1}\right)\right) \\
& =-m_{1}(K+\log (L)+B / L)
\end{aligned}
$$

where

$$
K=\log (d)+\log (2) / 2+\log \left(\rho_{c}\right) / \gamma-1, \quad L=c_{s, m_{1} / m_{2}} \nu m_{1}^{2}, \quad B=\pi / 2 .
$$

The contribution of $\mathcal{P}_{M}$. To take into account the effect of the factor $\mathcal{P}_{M}$ we need to identify the dominant term of $S_{A}$. From (29) and (27) it follows that the quotient of two consecutive 

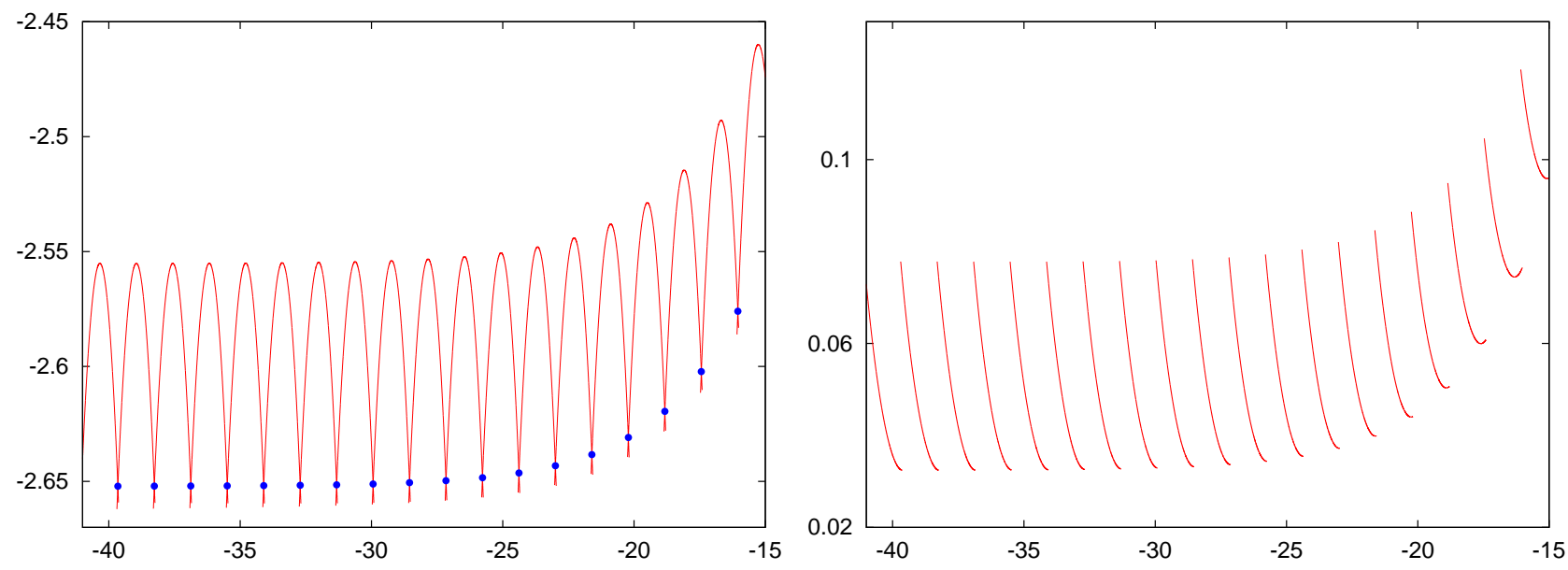

Figure 7: We consider $\gamma=(\sqrt{5}-1) / 2$ and $\epsilon=10^{-3}$. In both plots the horizontal variable is $\log _{2}(\nu)$. Left: $\sqrt{\nu} \log \left(C_{m_{1}, m_{2}}^{(1)} / \epsilon\right)$, the points correspond to the changes of dominant harmonic. The rightmost change corresponds to $m_{1}=55 \rightarrow m_{1}=89$, while the leftmost to $m_{1}=196418 \rightarrow$ $m_{1}=317811$. Right: $\sqrt{\nu} \log \left(\mathcal{P}_{M} \mathcal{P}_{Q}\right)$.

terms in the sum $S_{A}$ is

$$
\frac{A_{i}}{A_{i-1}}=\frac{m_{1}+2 i}{2\left(m_{1}+2 i-2\right)\left(m_{1}+i\right) i}\left(\frac{s}{\nu d}\right)^{2}\left(1+\left(m_{1}+2 i-2\right)^{2}(\nu / s)^{2}\right) .
$$

We look for the index $i$ corresponding to the term with maximum value of the sum $S_{A}$ for a fixed value of $\nu$. It is useful to introduce $I=i / m_{1}$ and look for the index $I$ instead. From (32), one gets

$$
\frac{A_{i}}{A_{i-1}}=\frac{1}{2 d^{2}}\left(\frac{1}{I+I^{2}}\left(\frac{s}{m_{1} \nu}\right)^{2}+\frac{1}{I+I^{2}}+4\right)\left(1+\mathcal{O}\left(m_{1}^{-1}\right)\right) .
$$

From this quotient one deduces that the sequence $\left\{A_{i}\right\}_{i}$ is increasing for small values of $i$ and it becomes decreasing for large values of $i$ provided $d>\sqrt{2}$ (recall that we choose $d=7$ in the concrete example). The maximum value is achieved when $A_{i} \approx A_{i-1}$. Then, ignoring the terms of relative value $\mathcal{O}\left(m_{1}^{-1}\right)$ one gets the following equation

$$
\left(2 d^{2}-4\right)\left(I^{2}+I\right)=1+\left(\frac{s}{\nu m_{1}}\right)^{2}
$$

from which one can determine the index $i=m_{1} I$ of the maximum term of $S_{A}$. Hence, taking into account the expression (29), the factor $\mathcal{P}_{M}$ is

$$
\mathcal{P}_{M}=\frac{m_{1} ! k}{d(\sqrt{2} w d)^{2 m_{1} I}\left(m_{1}(1+I)\right) !\left(m_{1} I\right) !} \Pi_{k}(w),
$$

where $k=m_{1}(1+2 I)$ and $w=\nu / s$. From the recurrence relation (27) one gets

$$
\log \left(\Pi_{k}(w)\right)=\sum_{j=k-2(-2) 0} \log \left(1+j^{2} w^{2}\right)
$$

where the index $j$ runs with step -2 (and finishes at $j=1$ whenever $k$ is odd). Approximating 


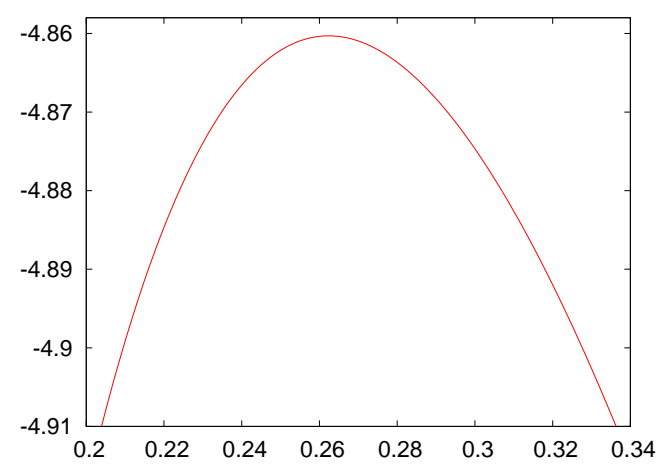

Figure 8: The universal function $\Psi_{1}(L)$ as a function of $L$.

the previous sum by an integral one has

$$
\begin{aligned}
\log \left(\Pi_{k}(w)\right) & \approx \frac{1}{2} \int_{0}^{k-1} \log \left(1+j^{2} w^{2}\right) d j=\frac{1}{4 w} \int_{0}^{(k-1)^{2} w^{2}} \log (1+z) \frac{d z}{\sqrt{z}} \\
& =\frac{1}{2 w}[\sqrt{z}(\log (1+z)-2)+2 \arctan (\sqrt{z})]_{0}^{(k-1)^{2} w^{2}} \\
& =\frac{k-1}{2}\left(\log \left(1+(k-1)^{2} w^{2}\right)-2\right)+\frac{\arctan ((k-1) w)}{w} .
\end{aligned}
$$

The definition of the universal function $\Psi_{1}(L)$. We define now the universal function $\Psi_{1}(L)$ from the previous contributions of $\mathcal{P}_{F}$ and $\mathcal{P}_{M}$. We will check below that the contribution of $\mathcal{P}_{Q}$ is not important in the sense that $\Delta F_{1}^{\{1\}} \approx \mathcal{P}_{F} \mathcal{P}_{M}$ is accurate enough to detect the changes of dominant harmonics.

First, we recall from (31) that $\log \left(\mathcal{P}_{F}\right) / m_{1} \approx-(K+\log (L)+B / L)$ with $K$ and $B$ independent of $L$. Let us denote by $\Psi_{1,1}(L)=-(K+\log (L)+B / L)$, and note that it slightly depends on $\gamma$ through $K$. Next, we obtain an approximation of $\log \left(\mathcal{P}_{M}\right) / m_{1}$ that only depends on $L=c_{s, m_{1} / m_{2}} \nu m_{1}^{2}$. Equation (34) can be rewritten as $\left(2 d^{2}-4\right)\left(I^{2}+I\right)-1=1 / L^{2}$, so that given $L$ we can obtain the index $I=I_{*}$ that determines $\mathcal{P}_{M}$. From (35), after skipping some constant terms and higher order terms in $m_{1}^{-1}$, one gets

$$
\begin{aligned}
\log \left(\mathcal{P}_{M}\right) / m_{1} \approx & -2 I_{*} \log (L d)-\left(1+I_{*}\right) \log \left(1+I_{*}\right)-I_{*} \log \left(I_{*}\right)+I_{*}(2-\log (2)) \\
& +\left(1+2 I_{*}\right)\left(\log \left(1+\left(\left(1+2 I_{*}\right) L\right)^{2}\right)-2\right) / 2+\arctan \left(\left(1+2 I_{*}\right) L\right) / L,
\end{aligned}
$$

where the terms of the first line come from the prefactor of $\Pi_{k}(w)$ in (35) and the terms of the second one are related to $\Pi_{k}(w)$ after taking logarithms. Let us denote by $\Psi_{1,2}(L)$ the right hand side of (37).

Now we define

$$
\Psi_{1}(L):=\Psi_{1,1}(L)+\Psi_{1,2}(L),
$$

which depends on the parameters $c$ and $d$ (and slightly on $\gamma$ through $K$ ) but does not depend explicitly on the approximant $m_{1} / m_{2}$ of $\gamma$. The universal function $\Psi_{1}(L)$ provides an approximation of $\sqrt{c_{s, m_{1} / m_{2}} \nu} \log \left(\left|\Delta F_{1}^{\{1\}}\right| / \epsilon\right)$ as a function of the parameter $L=c_{s, m_{1} / m_{2}} \nu m_{1}^{2}$. In Fig. 8 we show the function $\Psi_{1}(L)$ as a function of $L$. We can see that it has the properties described in Conjecture 7.1 
The factor $\mathcal{P}_{Q}$ plays no role. Here we check that $\mathcal{P}_{Q}$ becomes not relevant as $\nu \rightarrow 0$ or, equivalently, as $m_{1} \rightarrow \infty$. Ignoring the terms $\mathcal{O}\left(m_{1}^{-1}\right)$ in (33) we obtain

$$
\frac{A_{i}}{A_{i-1}}=\frac{1}{2 d^{2}\left(I+I^{2}\right)}\left((1+2 I)^{2}+L^{-2}\right) .
$$

This quotient depends on $L$ and $I$. For a fixed value of $L$ the quotient $A_{i} / A_{i-1}$ is a monotonically decreasing function of $I$ (and hence of $i$ ) independently of the value of $L$. Concretely one has

$$
\frac{\partial\left(A_{i} / A_{i-1}\right)}{\partial I}=-\frac{(1+2 I)\left(1+L^{-2}\right)}{2 d^{2}\left(I+I^{2}\right)^{2}} .
$$

Recall that $I_{*}$ is the value of $I$ giving the quotient $A_{i} / A_{i-1}$ closest to one (i.e. $I_{*}$ corresponds to the maximum term of the sum $S_{A}$ and, by definition, it determines the factor $\mathcal{P}_{M}$ ). For $\delta>0$ fixed, let $I_{ \pm}$the values of $I$ for which one has $A_{i} / A_{i-1}=1 \pm \delta$. One has

$$
I_{ \pm}=I_{*} \pm \partial I_{ \pm} /\left.\partial \delta\right|_{\delta=0} \delta+\mathcal{O}\left(\delta^{2}\right),
$$

and one checks that

$$
\partial I_{ \pm} /\left.\partial \delta\right|_{\delta=0}=\frac{2 d^{2}\left(I_{*}+I_{*}^{2}\right)}{\left(2 d^{2}-4\right)\left(1+2 I_{*}\right)}=\mathcal{O}(1)
$$

meaning that $\left|I_{+}-I_{-}\right|=\mathcal{O}(\delta)$. Since $I_{ \pm}=m_{1} i_{ \pm}$, it follows that $\left|i_{+}-i_{-}\right|=\mathcal{O}\left(m_{1} \delta\right)$.

We split the sum $S_{A}=\sum_{i \geq 0} A_{i}$ into three (say left/center/right) parts

$$
S_{A}=\sum_{i=0}^{i_{-}} A_{i}+\sum_{i=i_{-}}^{i_{+}} A_{i}+\sum_{i=i_{+}}^{\infty} A_{i}=S_{l}+S_{c}+S_{r} .
$$

We recall that $S_{A}=\mathcal{P}_{M} \mathcal{P}_{Q}$, where $\mathcal{P}_{M}=A_{i_{*}}$, where $i_{*}=m_{1} I_{*}$, hence

$$
\mathcal{P}_{Q}=\frac{1}{A_{i_{*}}}\left(S_{l}+S_{c}+S_{r}\right) \text {. }
$$

From $\left|i_{+}-i_{-}\right|=\mathcal{O}\left(m_{1} \delta\right)$, it follows that $S_{c}=\mathcal{O}\left(m_{1} \delta\right) A_{i_{*}}$. On the other hand, the terms in $S_{l}$ decay as $A_{i} \leq(1+\delta) A_{i-1}$. Hence, $S_{l} \leq A_{i_{*}} \sum_{i=0}^{i_{-}}(1+\delta)^{-i}=\mathcal{O}\left(A_{i_{*}} / \delta\right)$. Similarly, for $S_{r}$ one has $A_{i} \leq(1-\delta) A_{i-1}$, hence $S_{r}=\mathcal{O}\left(A_{i_{*}} / \delta\right)$. As a conclusion, one gets

$$
\mathcal{P}_{Q}=\mathcal{O}\left(m_{1} \delta\right)+\mathcal{O}\left(\delta^{-1}\right) .
$$

Taking, for example, $\delta=m_{1}^{-1 / 2}$ one gets $\mathcal{P}_{Q}=\mathcal{O}\left(m_{1}^{1 / 2}\right)$, meaning that the factor $\mathcal{P}_{Q}$ can be ignored compared with the exponentially small terms since its logarithm divided by $m_{1}$ is small compared with the other terms in $\Psi_{1}$.

The analogous function $\Psi_{2}(L)$. For a fixed $m_{1} / m_{2} \in \mathbb{Q}$ we define the function $\Psi_{2}(L)$ as

$$
\Psi_{2}(L)=\Psi_{1}(L)-\frac{\sqrt{L}}{m_{1}} \log (L) .
$$

From Proposition 7.1 one has that

$$
\Psi_{2}(L) \approx \sqrt{c_{s, m_{1} / m_{2}} \nu} \log \left(C_{m_{1}, m_{2}}^{(2)}\right) .
$$

Assume that we are interested in the functions $\Psi_{1}(L)$ and $\Psi_{2}(L)$ for values of $L \in\left[L_{-}, L_{+}\right]$ around their maxima. Then, the relation (39) shows that $\Psi_{2}(L)$ tends to $\Psi_{1}(L)$ as $\nu \rightarrow 0$, uniformly in $\left[L_{-}, L_{+}\right]$. 


\subsection{The changes in the dominant harmonic of the splitting function}

Several properties can be analysed from the derived universal functions $\Psi_{1}$ and $\Psi_{2}$.

First we look for the changes of the dominant harmonic in $\Delta F_{1}^{\{1\}}$ as $\nu$ varies. We expect that for most of the values of $\nu$ there is one dominant harmonic. However, for some values of $\nu$ different harmonics can be of the same order of magnitude. Our aim is to determine, for a given $\nu$ small enough, which is (are) the dominant harmonic(s).

Some general comments are in order. As already said and according to (28) (resp. (30)), for $\nu$ small enough one expects the dominant harmonic(s) of $\Delta F_{1}^{\{1\}}$ (resp. $\Delta F_{2}^{\{1\}}$ ) to be related with the best approximants of $\gamma$. That is, to get the dominant harmonic it is enough to compare the harmonics associated to best approximants $m_{1} / m_{2}$ of $\gamma$. Below we will restrict to best approximants and we will compare the functions $\Psi_{1}$ associated to them. However, not all the harmonics associated to best approximants become a dominant harmonic. Several examples will be given in Section 7.6. Finally, we note that, assuming that the amplitudes of the harmonics of the Poincaré-Melnikov integral decay in an exponential way as in Remark 5.1, at least one of every two consecutive best approximants of $\gamma$ becomes the dominant harmonic of $\Delta F_{i}^{\{1\}}$, $i=1,2$, for a suitable range of $\nu$. In Appendix E we consider that problem assuming two small consecutive quotients between two large quotients of the CFE of $\gamma$. For a more general discussion see [9].

To determine which of the best approximants is associated to the dominant harmonic requires to know the constants $c_{s, m_{1} / m_{2}}$ to be able to compare the corresponding functions $\Psi_{1}$. If moreover one wants to look for the asymptotic behaviour of the changes of dominant harmonic as $\nu \rightarrow 0$ one needs an asymptotic description of the values of $c_{s, m_{1} / m_{2}}$. Next subsections deal with this question.

\subsubsection{The golden mean frequency.}

For simplicity, first we consider $\gamma$ to be a quadratic irrational so that its CFE is periodic. We shall prove in Lemma 7.2 that, in this case, the values of the constants $c_{s, m_{1} / m_{2}}$ associated to the best approximants of $\gamma$ are (asymptotically, as the order of the best approximant tends to infinity) also periodic. Moreover, for concreteness, we focus on $\gamma=(\sqrt{5}-1) / 2$ but other quadratic irrational numbers can be similarly handled.

As we shall discuss in Section 7.6.1, for $\gamma=(\sqrt{5}-1) / 2$, one has $c_{s, n} \rightarrow \sqrt{5}(1+\gamma)=3+\gamma$ when considering best approximants of $\gamma$ and as the order of the best approximant tends to infinity. The best approximants are quotients of consecutive Fibonacci numbers. It turns out that all best approximants are visible as a dominant harmonic in a corresponding interval of $\nu$. We look for the sequence of values $\nu_{j}$ of $\nu$ for which the changes of dominant harmonic take place, see Fig. 7 left. Assume that the $j$-th best approximant of $\gamma$ dominates at a specific value of $\nu=\nu_{1}^{*}$. We first use the approximation $\Delta F_{1}^{\{1\}} \approx \epsilon \mathcal{P}_{F}\left(m_{1}\right)$ where $m_{1}$ is the numerator of the $j$-th best approximant. Assume that for $\nu=\nu_{0}^{*}<\nu_{1}^{*}$ the dominant harmonic corresponds to the $(j+1)$-th best approximant of $\gamma$. Then there is a value $\nu=\nu_{j}$, corresponding to the change $m_{1} \rightarrow(1+\gamma) m_{1}$ of dominant harmonic, for which $\log \left(\mathcal{P}_{F}\left(m_{1}\right)\right)=\log \left(\mathcal{P}_{F}\left((1+\gamma) m_{1}\right)\right)$. This condition leads to the following equation for $L$

$$
L=\frac{\pi \gamma /(2(1+\gamma))}{2(1+\gamma) \log (1+\gamma)+K \gamma+\gamma \log (L)} .
$$


This equation, which is independent of $m_{1}$, can be solved by numerical iteration and one obtains $L=L_{l} \approx 0.1690224$ for the values $c=5, d=7$ in our perturbation. This implies that asymptotically $\nu_{j+1} \approx \gamma^{2} \nu_{j}$. Indeed, from $m_{2} \approx m_{1}(1+\gamma)$ it follows that $L=\nu_{j} m_{1}^{2} c_{s, m_{1} / m_{2}} \approx$ $\nu_{j+1} m_{1}^{2}(1+\gamma)^{2} c_{s, m_{1} / m_{2}}$ and then $\nu_{j+1} \approx \gamma^{2} \nu_{j}$. Accordingly, this agrees with Fig. 7 left where the values $\log _{2}\left(\nu_{j}\right)$ tend to be, as $\nu \rightarrow 0$, separated by $2 \log _{2}(\gamma) \approx-1.38848$.

More concretely, let $F_{j}$ denote the Fibonacci sequence starting with $F_{1}=1, F_{2}=2, F_{3}=3, \ldots$ We can compute the values $\nu=\nu_{j}$ where $\nu_{j}$ corresponds to the change $m_{1}=F_{j} \rightarrow m_{1}=F_{j+1}$. With this notation the blue points in Fig. (7left correspond to the values of $\log _{2}\left(\nu_{j}\right)$ for $9 \leq j \leq$ 26. Moreover, one has $\nu_{j} \sim \gamma^{2 j} \hat{K}$, for some $\hat{K}$. In Fig. 9 we represent $\nu_{j} \gamma^{-2 j}$ as a function of $j$. We see that, for $j$ large enough, it tends to the constant $\hat{K} \approx 0.0850$.

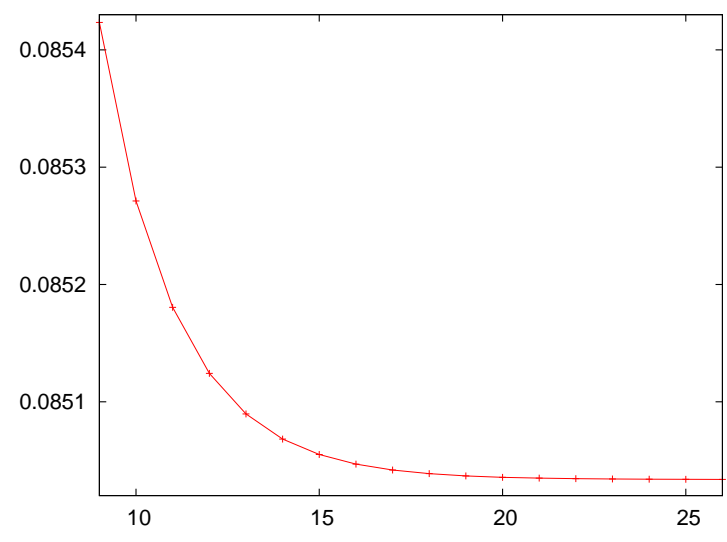

Figure 9: We represent $\nu_{j} \gamma^{-2 j}$, where $\nu_{j}$ are the values where a change of dominant harmonic has been numerically detected, as a function of the index $j$ of the Fibonacci sequence $F_{j}$ (see text for details).

Let us describe a more general methodology to look for the changes of dominant harmonic which takes into account the corrections due to the factor $\mathcal{P}_{M}$. Since for $\gamma=(\sqrt{5}-1) / 2$ one has $c_{s, m_{1} / m_{2}}=c_{s, n} \rightarrow 3+\gamma \approx 3.618034$ we introduce $\tilde{L}=L / c_{s, m_{1} / m_{2}}$ and we consider $\hat{\Psi}_{1}(\tilde{L}):=\Psi_{1}(\tilde{L}) / \sqrt{c_{s, m_{1} / m_{2}}}$. In Fig. 10 we represent the leftmost five peaks of Fig. 7 left as a function of the parameter $\tilde{L}$. They correspond to $m_{1}=46368,75025,121393,196418,317811$. Also, in blue, we represent the function $\hat{\Psi}_{1}(\tilde{L})$. We see in the right plot that, as $\nu$ decreases to 0 , the curves tend to $\hat{\Psi}_{1}(\tilde{L})$.

In Fig. 11 we represent the function $\hat{\Psi}_{1}(\tilde{L})$ as a function of $\log (\tilde{L})$. The maximum of $\hat{\Psi}_{1}(\tilde{L})$ is $\approx-2.555210$, in good agreement with the numerical values shown in Fig. 10 and in Fig. 7 left. It is achieved for $\tilde{L} \approx 0.072529$. After a change of coordinates the function $\hat{\Psi}_{1}(\tilde{L})$ behaves as $-\log (\cosh (\tilde{L}))$, see [1, [5].

Let us consider two values of $\tilde{L}$, say $\tilde{L}_{1}$ and $\tilde{L}_{2}, \tilde{L}_{1}<\tilde{L}_{2}$, corresponding to different harmonics. Assume that for $\nu>0$ small enough these harmonics are related to two consecutive best approximants of $\gamma$, say $m_{1} / m_{2}$ and $m_{2} / m_{3}$ (the numerators $m_{1}$ and $m_{2}$ are two consecutive Fibonacci numbers). Assume that the change of harmonic takes place at $\nu=\nu_{0}^{*}$ then $\tilde{L}_{1}=m_{1}^{2} \nu_{0}^{*}$, $\tilde{L}_{2}=m_{2}^{2} \nu_{0}^{*}$ and $\hat{\Psi}_{1}\left(\tilde{L}_{1}\right)=\hat{\Psi}_{1}\left(\tilde{L}_{2}\right)$. Moreover, if $m_{1}$ is large, then one has $m_{2} \approx(1+\gamma) m_{1}$ and therefore $\tilde{L}_{2} \approx(1+\gamma)^{2} \tilde{L}_{1}$. One obtains $\tilde{L}_{l} \approx 0.044524$ as the asymptotic value of $\tilde{L}$ where the change takes place. Notice that $L=L_{l} \approx 0.16109$, which is very close to the value of $\tilde{L}_{l}$ obtained above using just $\mathcal{P}_{F}$. One has $\hat{\Psi}_{1}\left(\tilde{L}_{l}\right) \approx-2.652115$, which is represented as an horizontal line in Fig. 11. We conclude that at the value $\nu=\nu_{j} \approx \tilde{L}_{l} / F_{j}^{2}$ takes place the change 

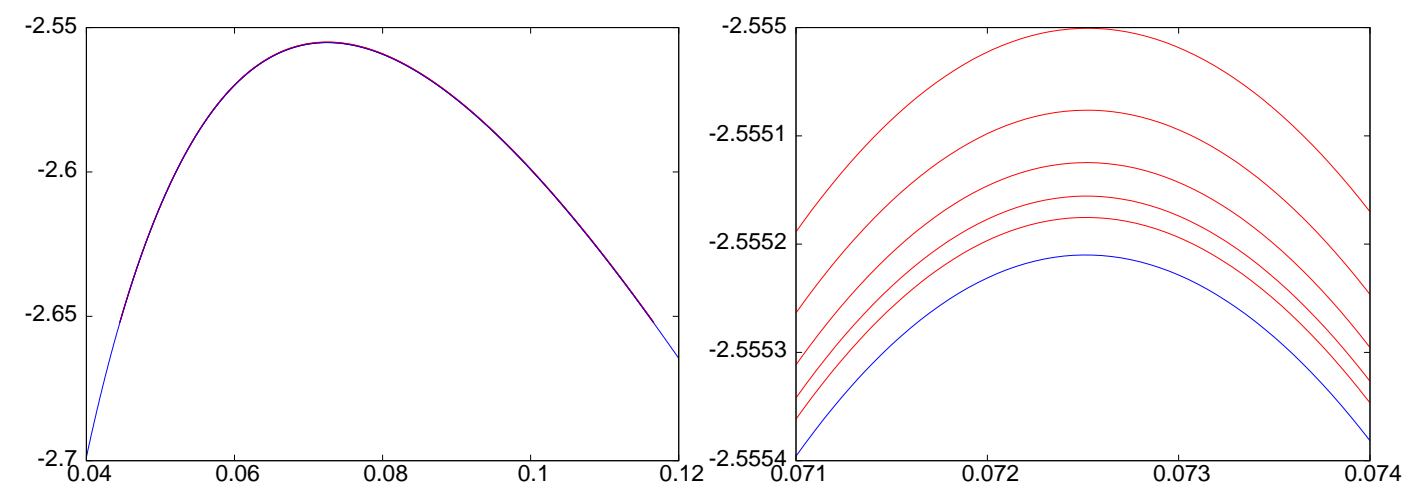

Figure 10: Left: The five leftmost peaks of Fig. 7 as a function of $\tilde{L}$ (in red). The function $\hat{\Psi}_{1}(\tilde{L})$ is also shown (in blue). All of them almost coincide at this scale. Right: Magnification of the central zone of the left plot. We see that the peaks move down as $\nu$ decreases (and $m_{1}$ increases). They tend to $\hat{\Psi}_{1}(\tilde{L})$.

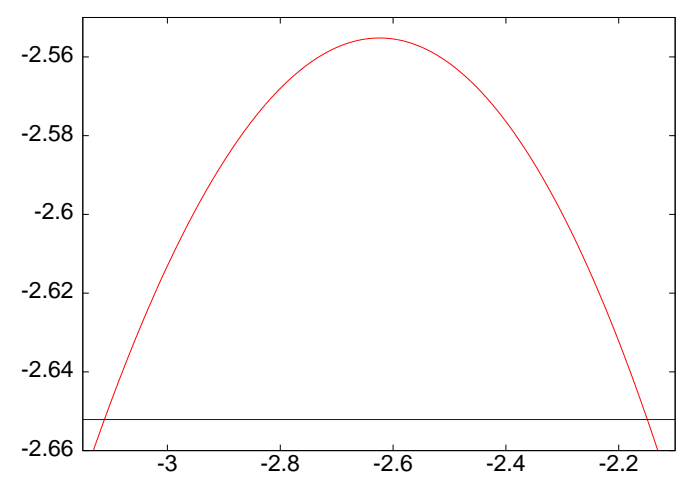

Figure 11: We depict $\hat{\Psi}_{1}(\tilde{L})$ as a function of $\log (\tilde{L})$ for $\gamma=(\sqrt{5}-1) / 2$ (see text for details).

$m_{1}=F_{j} \rightarrow m_{1}=F_{j+1}$ of dominant harmonic of $\Delta F_{1}^{\{1\}}$.

In Table 1 we can see that, for large range intervals of $\nu$, both $\Delta F_{1}^{\{1\}}$ and $\Delta F_{2}^{\{1\}}$ have the same dominant harmonic. Indeed, relation (39) implies, in particular, that the changes of dominant harmonic in $\Delta F_{1}^{\{1\}}$ and in $\Delta F_{2}^{\{1\}}$ tend to coincide as $\nu \rightarrow 0$. Concretely, denote by $\nu_{j}^{(i)}$ the sequence of values of $\nu$ for which the dominant harmonic of $\Delta F_{i}^{\{1\}}$ changes, the values of $\nu_{j}^{(1)}$ have been determined in Section 7.4.1. To look for the values $\nu_{j}^{(2)}$ we consider the condition $\Psi_{2}\left(L_{1}\right)=\Psi_{2}\left(L_{2}\right)$ with $L_{2}=L_{1}(1+\gamma)^{2}$ which, by (39), is equivalent to

$$
\Psi_{1}\left(L_{1}\right)=\Psi_{1}\left(L_{1}(1+\gamma)^{2}\right)-\frac{\sqrt{L_{1}}}{m_{1}}\left(\gamma \log \left(L_{1}\right)+2(1+\gamma) \log (1+\gamma)\right) .
$$

Note that, since $L=c_{s, m_{1} / m_{2}} \nu m_{1}^{2}$, when $\nu \rightarrow 0$ we recover the condition that determines the values $\nu_{j}^{(1)}$. One has $\nu_{j}^{(2)}=\tilde{L}_{l}^{(2)} / F_{j}^{2}$, where $\tilde{L}_{l}^{(2)}=\tilde{L}_{l}+\mathcal{O}(\sqrt{\nu})$, being $\tilde{L}_{l} \approx 0.044525$. The values of $\nu_{j}^{(1)}$ and $\nu_{j}^{(2)}$, corresponding to the changes of dominant harmonic in $\Delta F_{1}^{\{1\}}$ and $\Delta F_{2}^{\{1\}}$, respectively, are displayed in Table 2, We have considered the range $\log _{2}(\nu) \in[-24,-16]$. We refer to Fig. 6] left where the computation of the amplitude of the splitting for this range of values of $\nu$ is shown. The best approximant $N_{j} / D_{j}=F_{j} / F_{j+1}$ corresponds to a dominant harmonic 


\begin{tabular}{|c|c|c|c|c|c|}
\hline$N_{j}$ & $N_{j+1}$ & $\log _{2}\left(\nu_{j}^{(1)}\right)$ & $\log _{2}\left(\nu_{j}^{(2)}\right)$ & $\nu_{j}^{(1)}-\nu_{j}^{(2)}$ & Coeff \\
\hline 55 & 89 & -16.04563135 & -16.05223394 & $0.675040 \mathrm{E}-07$ & 1.191635 \\
89 & 144 & -17.43664042 & -17.44071697 & $0.159057 \mathrm{E}-07$ & 1.190968 \\
144 & 233 & -18.82665512 & -18.82917332 & $0.375102 \mathrm{E}-08$ & 1.190692 \\
233 & 377 & -20.21609319 & -20.21764898 & $0.884894 \mathrm{E}-09$ & 1.190469 \\
377 & 610 & -21.60516252 & -21.60612386 & $0.208812 \mathrm{E}-09$ & 1.190355 \\
610 & 987 & -22.99400932 & -22.99460338 & $0.492817 \mathrm{E}-10$ & 1.190280 \\
\hline
\end{tabular}

Table 2: Values of $\nu_{j}^{(1)}$ and $\nu_{j}^{(2)}$ for which the change from the dominant harmonic related to the approximant $N_{j} / D_{j}$ to $N_{j+1} / D_{j+1}$ takes place. The last column displays the value of the coefficient Coeff $\approx\left(\nu_{j}^{(1)}-\nu_{j}^{(2)}\right) / \nu_{m}^{3 / 2}, \nu_{m}=\left(\nu_{j}^{(1)}+\nu_{j}^{(2)}\right) / 2$.

for $\nu_{j}=\mathcal{O}\left(1 / F_{j}^{2}\right)$. Hence $\nu_{j}^{(1)}-\nu_{j}^{(2)}=\tilde{L}_{l} / F_{j}^{2}-\left(\tilde{L}_{l}+\mathcal{O}\left(\sqrt{\nu_{j}}\right)\right) / F_{j}^{2}=\mathcal{O}\left(\nu_{j} \sqrt{\nu_{j}}\right)$, as it is observed in the last column of Table 2 .

We remark that the previous comments assert that $\nu_{j}^{(1)}$ and $\nu_{j}^{(2)}$, corresponding to changes of dominant harmonic in $\Delta F_{1}^{\{1\}}$ and $\Delta F_{2}^{\{1\}}$, tend to coincide as $\nu \rightarrow 0$. For values of $\nu \in I_{j}=$ $\left[\nu_{j}^{(2)}, \nu_{j}^{(1)}\right]$ the dominant harmonic of each splitting function is different. This has some dynamical consequences: according to Appendix $\mathrm{D}$ one expects to have a faster diffusion process in phase space (but taking place in exponentially large times!) for values of $\nu \in I_{j}$ rather than for values of $\nu$ outside the union of the intervals $I_{j}$. Numerical massive investigations of the diffusion phenomena taking place for the example considered in this work and for small enough values of $\nu$ so that the limit behaviour can be observed would require a huge (nowadays prohibitive!) amount of computing time. Nevertheless, we believe that some numerical explorations of this model for moderate values of $\nu$ are of much interest. We postpone them for future works.

\subsubsection{A general frequency $\gamma$}

The same strategy can be used to look for values $\nu_{j}$ for which there is a change of dominant harmonic of $\Delta F_{1}^{\{1\}}$ (and of $\Delta F_{2}^{\{1\}}$ ) for general $\gamma$. Consider approximants $m_{1} / m_{2}$ and $n_{1} / n_{2}$ of $\gamma$ such that the related harmonics become dominant for $\Delta F_{1}^{\{1\}}$ (similar for $\Delta F_{2}^{\{1\}}$ ) in adjacent intervals of $\nu$. The change of dominant harmonic for $\Delta F_{1}^{\{1\}}$ takes place for $\nu$ such that $\Psi_{1}\left(L_{1}\right)=\Psi_{1}\left(L_{2}\right)$, where $L_{1}=c_{s, m_{1} / m_{2}} \nu m_{1}^{2}$ and $L_{2}=c_{s, n_{1} / n_{2}} \nu m_{1}^{2}$. Using that $L_{2}=L_{1} n_{1}^{2} c_{s, n_{1} / n_{2}} /\left(m_{1}^{2} c_{s, m_{1} / m_{2}}\right)$ the previous equation can be solved for $L_{1}$ (e.g. numerically by simple iteration) to obtain the values of $\nu=\nu_{j}$ corresponding to the changes.

As an illustrative example, we show in Fig. 12 the results for the transcendental frequency number $\gamma=e-2$. From its CFE properties it follows that the constants $c_{s, m_{1} / m_{2}}$ become unbounded, see details in Section 7.6.2. On the other hand, we see in the figure that all the harmonics related to best approximants become dominant in a suitable range of $\nu$. We remark that for other $\gamma$ it might happen that some best approximants will not be related to a dominant harmonic of $\Delta F_{1}^{\{1\}}$ (see examples in Section [7.6). Concretely, for $\gamma=e-2$, we show in Fig. 12 the functions $\Psi_{1}$ in blue lines and the points that correspond to the values of $\nu$ where a change of the dominant harmonic takes place. These values are obtained by comparing the functions $\Psi_{1}$ for different approximants as explained above in this section. As an extra check, we have compared the values of $\nu$ obtained by the previous procedure with the corresponding values obtained if one computes the contribution of each harmonic $m_{1} / m_{2}$ using the complete expression (28) for 
$C_{m_{1}, m_{2}}$. These contributions are shown in red lines in the figure. We see that the blue lines are good enough approximations of the red ones for $\nu$ small enough. Moreover the values of $\nu$ are almost coincident even for the rightmost part of the figure where the agreement between the blue and red curves is not so good.

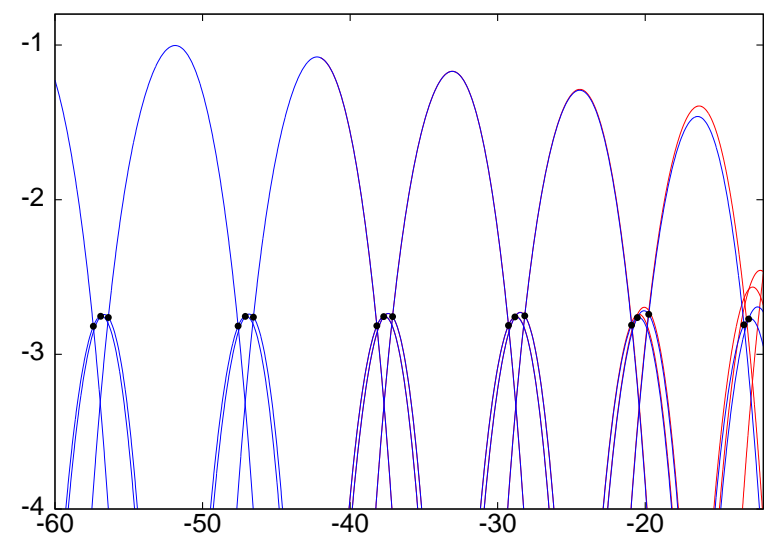

Figure 12: For $\gamma=e-2$ we show the contribution of the different harmonics, the range of $\nu$ where they become dominant and the changes. The horizontal axis corresponds to $\log _{2}(\nu)$. In red we depict $\sqrt{\nu} \log \left(C_{m_{1}, m_{2}}\right)$. In blue the functions $\Psi_{1}(L) / \sqrt{c_{s, m_{1} / m_{2}}}$ obtained for the corresponding best approximants. The points correspond to the values of $\nu$ where there is a change of dominant harmonic. They are computed using the functions $\Psi_{1}(L)$. Note that for $\log _{2}(\nu)<-25$ both red and blue curves become almost coincident.

\subsection{The effect of non-dominant terms of the splitting function}

To find the dominant terms of the splitting $\Delta F_{1}^{\{1\}}$ we have considered values of $\nu$ small enough (fixed) and have looked for the values of $m_{1}$ for which $L=c_{s, m_{1} / m_{2}} \nu m_{1}^{2}$ is the closest to the maximum of $\Psi_{1}(L)$. This term (or these terms if, for example, we are close to a change of dominant harmonic) gives the maximum contribution to the Melnikov function $\Delta F_{1}^{\{1\}}$ in (25). However, to assert that the splitting Melnikov function $\Delta F_{1}^{\{1\}}$ is of the order of this/these dominant terms there are some details to be checked. As said in the Introduction, a theoretical proof must consider the effect of all the harmonics of the splitting function, bound the effect of the ones related to approximants which are not best approximants, and bound the effect of the best approximants which are non-dominant (for the values of $\nu$ considered). In particular, one has to address the following questions.

1. For a fixed $\nu$ we look for $m_{1}$ giving the most important terms in $\Delta F_{1}^{\{1\}}$. Which is the effect of the other terms associated to best approximants for this value of $\nu$ ?

2. Of course there are other approximants of $\gamma$ which are not best approximants. We call them "subapproximants". Which is their contribution to $\Delta F_{1}^{\{1\}}$ ? Which are the corresponding constants $c_{s, m_{1} / m_{2}}$ related to each family of subapproximants and which is their contribution to $\Delta F_{1}^{\{1\}}$ ?

3. Looking at the expression (25) of $\Delta F_{1}^{\{1\}}$ we see that values of $k, i, j$ for which $s$ is large correspond to terms which make a small contribution to the total sum. But there are infinitely many of these terms. How to bound their total contribution? 
Even if we are not going to address these questions formally, we want to provide an idea of how useful can be the universal function $\Psi_{1}$ to investigate such questions. For concreteness we focus on $\gamma=(\sqrt{5}-1) / 2$. We recall that in this case one has $c_{s, n} \rightarrow 3+\gamma$ as $n \rightarrow \infty$ (see Section 7.6.1). We proceed as follows.

1. To evaluate the function $\Psi_{1}(L)$ we consider the algorithm introduced in Section 7.4. Recall that $\Psi_{1}$ depends on $\gamma, c_{s, m_{1} / m_{2}}, c$ and $d$ but not on $\nu$.

2. We compute the maximum of $\Psi_{1}(L)$. We denote by $\tilde{L}_{M}$ the value of $\tilde{L}=L / c_{s, m_{1} / m_{2}}$ for which the maximum is attained.

3. We take $\nu$ small enough and we look for the integer $m_{1}$, among the numerators of the best approximants, closest to $\sqrt{\tilde{L}_{M} / \nu}$. Maybe there are two integer values at a similar distance and a bifurcation takes place because the dominant harmonic of $\Delta F_{1}^{\{1\}}$ changes. For $\gamma=(\sqrt{5}-1) / 2$ this happens whenever $\Psi_{1}(L)=\Psi_{1}\left(L(1+\gamma)^{2}\right)$.

4. If for the chosen value of $\nu$ there is an integer $m_{1}$ for which $\tilde{L}=\nu m_{1}^{2}=\tilde{L}_{M}$, then we have to check that the value of $\Psi_{1}$ at $\tilde{L}=\tilde{L}_{M k}:=\tilde{L}_{M}(1+\gamma)^{k}$ for $k= \pm 2, \pm 4, \ldots$ is small enough.

5. If $\nu=\nu_{B}$ corresponds to a bifurcation then $\Psi_{1}\left(\tilde{L}_{B}\right)=\Psi_{1}\left(\tilde{L}_{B}(1+\gamma)^{2}\right)$ for $\tilde{L}_{B}=\nu m_{1}^{2}$.

6. It might be also interesting to look for values of $\nu$ for which there is a dominant harmonic but there is a change of subdominant. This happens for $\tilde{L}=\tilde{L}_{C}$ such that $\Psi_{1}\left(\tilde{L}_{C}(1+\gamma)^{2}\right)=$ $\Psi_{1}\left(\tilde{L}_{C} /(1+\gamma)^{2}\right)$.

We note that we have performed all the computations for $c=5$ and $d=7$. What happens in the limit cases, that is, either for $d \rightarrow \sqrt{2}$ as a function of $c$ or for $c \rightarrow 1$ as a function of $d$ ? Note that when $c \rightarrow 1$ the function $f(\theta)$, see (15), tends to be unbounded as well as its Fourier coefficients (16). The same thing happens for the function $g\left(y_{1}\right)$ when $d \rightarrow \sqrt{2}$ and its power expansion.

In Fig. 13 we summarize some data obtained by the implementation of the previous items. Concretely, in Fig. 13 top left we show the points $\tilde{L}_{S}$ where $S=M, B, C$. The points with subscript + and $++\left(\right.$ resp. - and --) denote the values of $\tilde{L}_{S}$ for the next and the second next approximants to $\gamma$. Since we are dealing with the golden frequency $\gamma$ we have considered the normalized function

$$
\hat{\Psi}_{1}(\tilde{L})=\Psi_{1}(\tilde{L}) / \sqrt{c_{\infty}}, \quad \text { being } c_{\infty}=3+\gamma \text { the limit value of }\left\{c_{s, n}\right\}_{n},
$$

and we represent $\hat{\Psi}_{1}(\tilde{L})$ as a function of $\log (\tilde{L})$. We also show the same function translated to the right and to the left by $2 \log (1+\gamma)$. These correspond to the functions $\Psi_{1}$ associated to the previous and next best approximants of $\gamma$. The top left plot corresponds to $c=5, d=7$. In the top center plot we represent the same as in the top left one, but for values $c=1.1, d=1.5$ close to the limit.

In the top right plot of Fig. 13 we represent $\log \left(-\hat{\Psi}_{1}(\tilde{L})\right)$ as a function of $\log \tilde{L}$ for $c=5, d=7$, and we check that $\log \left(-\hat{\Psi}_{1}(\tilde{L})\right)$ behaves as $|\log \tilde{L}| / 2$ as follows from the expressions for $I, \mathcal{P}_{F}$, $\mathcal{P}_{M}$ and $\Psi$, in (34), (31), (37) and (38), respectively. In the logarithmic scale used in the plot we clearly observe that, after shifting the origin and scaling coordinates, $\hat{\Psi}_{1}$ behaves as $\log (\cosh (\tilde{L}))$. 
The dependence of the maximum value of $\hat{\Psi}_{1}(\tilde{L})$ as a function of $(c, d)$ forms the surface shown in the bottom row of Fig. 13. We recall our notation: the maximum of $\hat{\Psi}_{1}(\tilde{L})$ is achieved at $\tilde{L}=\tilde{L}_{M}$. As expected all the maxima are negative values.
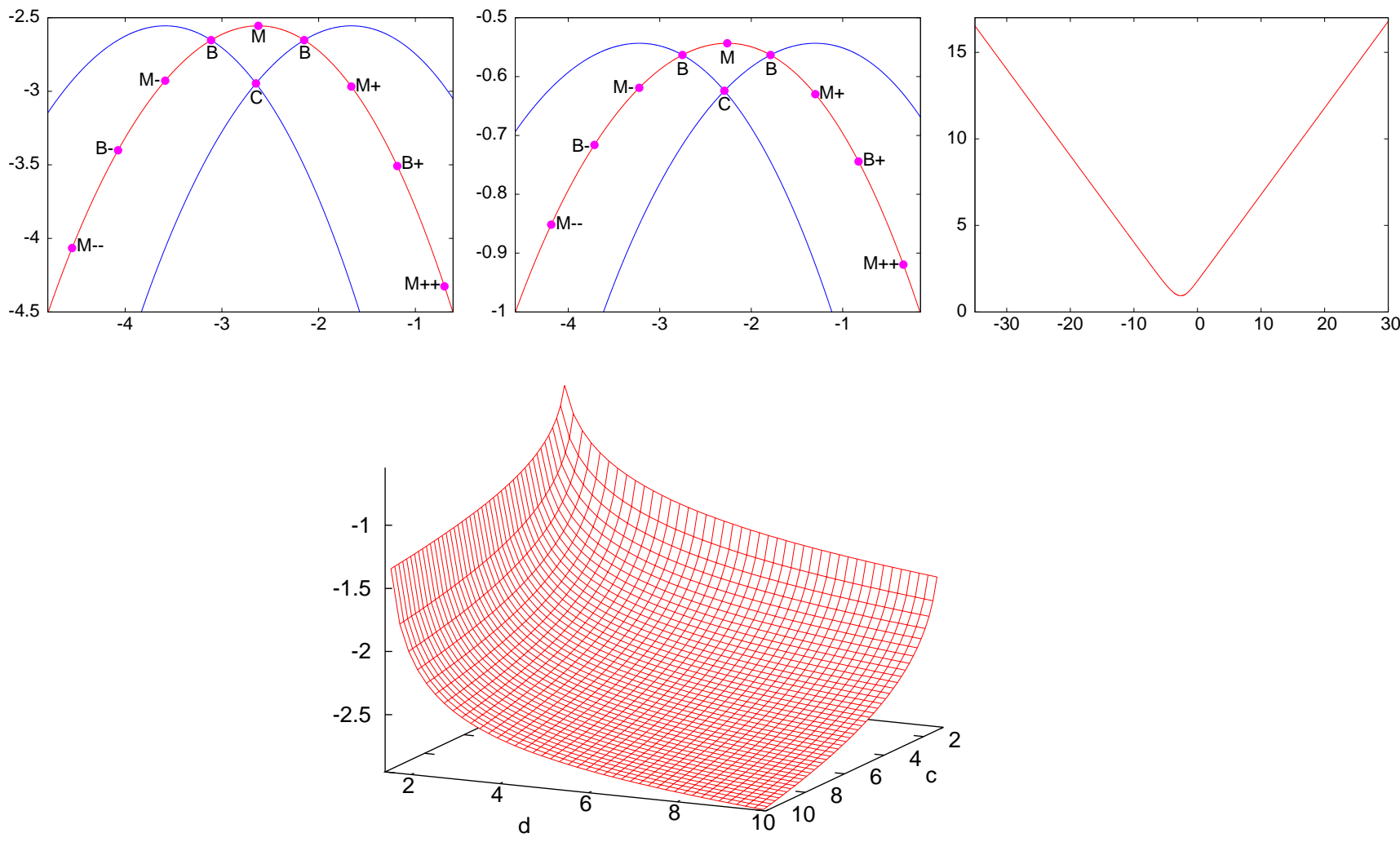

Figure 13: $\gamma=(\sqrt{5}-1) / 2$. Top left: $\hat{\Psi}_{1}(\tilde{L})$ as a function of $\log (\tilde{L})$ for the values $c=5, d=7$. Also we show the same function translated to the left and to the right by $2 \log (1+\gamma)$. The marked points are: $M$ for maximum and then $M_{++}, M_{+}, M_{-}, M_{--}$for the $m_{1}$ values of the previous and next approximants; $B$ for the change of dominant harmonic and then $B_{+}, B_{-}$for the nearby approximants too; $C$ for the subdominant harmonic change. Top center: the same as in the top left but for $c, d$ values close to the limit: $c=1.1, d=1.5$. Top right: for $c=5, d=7$ we show $\log \left(-\hat{\Psi}_{1}(\tilde{L})\right)$ as a function of $\log (\tilde{L})$. Bottom: Maxima of $\hat{\Psi}_{1}(\tilde{L})$ as a function of $(c, d)$.

\subsection{The splitting function for different frequencies}

In this section we illustrate what happens for several frequencies $\gamma$. We show some computations for concrete cases, including the golden mean, for comparison, in Fig. 14. The sequence of dominant harmonics and the values $\nu=\nu_{j}$ at which the change of dominant harmonic takes place depend on the CFE and not only on the Diophantine properties of $\gamma$. In Fig. 14 we represent the contributions $C_{m_{1}, m_{2}}$ to $\Delta F_{1}^{\{1\}} / \epsilon$ as a function of $\log _{2}(\nu)$ for different values of $\gamma$. The results for $\gamma=(\sqrt{5}-1) / 2$ are shown in the top left plot (case 0$)$. Concretely, we represent the contributions $C_{m_{1}, m_{2}}$ corresponding to the approximants of the golden frequency with $m_{1}$ between 21 and 514229. Compare with Fig. 7 left. Note that all the approximants become dominant in a suitable range of $\nu$. However, as can be seen in the plots, this does not happen for other frequencies $\gamma$. For concreteness, below we consider the following cases (the notation $10 \times 1$ in the CFEs below denotes ten consecutive quotients equal to one). 
Case $0: \gamma=(\sqrt{5}-1) / 2=[1,1,1,1,1, \ldots] \approx 0.618033988749894848204$.

Case $1: \gamma=(55(1+b)+34) /(89(1+b)+55)$ with $b=(\sqrt{122}-10) / 11$,

hence $\gamma=[10 \times 1,1,10,1,1,10,1,1,10,1, \ldots] \approx 0.6180512268192526496794$.

Case $2: \gamma=(55(1+b)+34) /(89(1+b)+55)$ with $b=(\sqrt{140}-10) / 20$,

hence $\gamma=[10 \times 1,1,10,1,10,1,10,1,10 \ldots] \approx 0.6180513744611582707944$.

Case 3: $\gamma=[10 \times 1,2,3,4,5,6,7,8,9,10, \ldots] \approx 0.6180206632934375446297$.

For each one of the previous cases, we list the consecutive numerators of the approximants of $\gamma$ for which the corresponding harmonic term of the splitting function become dominant (in a suitable range of $\nu$ ).

Case 0: 21, 34, 55, 89, 144, 233, 377, 610, 987, 1597, 2584, 4181, 6765, 10946, 17711, 28657, $46368,75025,121393,196418,317811,514229$.

Case 1: 21,34, 89, 945, 1034, 1979, 20824, 22803, 43627, 459073, 502700.

Case 2: 21,34, 89, 1034, 12319, 146794 .

Case 3: 21, 34, 55, 144, 487, 2092, 10947, 67774, 485365.

The contributions of the harmonic terms related to consecutive best approximants to the total splitting are shown in Fig. 14, In order to explain the results displayed in the figure for different frequencies $\gamma$, we investigate the Diophantine properties of $\gamma$ and relate them to the properties of the constants $c_{s, m_{1} / m_{2}}$.

\subsubsection{Periodicity of the constants $c_{s, m_{1} / m_{2}}$ for quadratic irrational frequencies}

First, it turns out that for quadratic $\gamma \in \mathbb{R} \backslash \mathbb{Q}$ the constants $c_{s, m_{1} / m_{2}}$ tend to be periodic when $\nu \rightarrow 0$. This is a consequence of the basic CFE property in Lemma 7.1 below.

Let $\left\{q_{j}\right\}_{j \geq 0}$ be an infinite or finite sequence of natural numbers, with $q_{0} \geq 0$ and $q_{j} \geq 1$ for $j \geq 1$, which defines a CFE of a real number in the usual way. Given a frequency $\gamma=\left[q_{0} ; q_{1}, q_{2}, \ldots\right]=$ $q_{0}+\frac{1}{q_{1}+\frac{1}{q_{2}+\ldots}}$, denote by $N_{n} / D_{n}=\left[q_{0} ; q_{1}, \ldots, q_{n}\right], n \geq 0$, the $n$-th order approximant of $\gamma$. Introducing $N_{-1}=1, D_{-1}=0$, the following basic properties hold (see for example [14] for proofs). For all $n \geq 1$,

(i) $N_{n}=q_{n} N_{n-1}+N_{n-2}, \quad D_{n}=q_{n} D_{n-1}+D_{n-2}$.

(ii) $\left|D_{n} N_{n-1}-D_{n-1} N_{n}\right|=1$.

(iii) If $\beta_{n}=\left[0 ; q_{n+1}, \ldots\right]$ then $\gamma=\left[q_{0} ; q_{1}, q_{2}, \ldots, q_{n-1}, q_{n}+\beta_{n}\right]=\frac{N_{n}+\beta_{n} N_{n-1}}{D_{n}+\beta_{n} D_{n-1}}$.

(iv) $\frac{D_{n-1}}{D_{n}}=\left[0 ; q_{n}, q_{n-1}, \ldots, q_{1}\right]$.

We introduce the notation $q_{+, n}=\left[q_{n+1} ; q_{n+2}, \ldots\right]$ and $q_{-, n}=\left[q_{n} ; q_{n-1}, \ldots, q_{1}\right]$. 

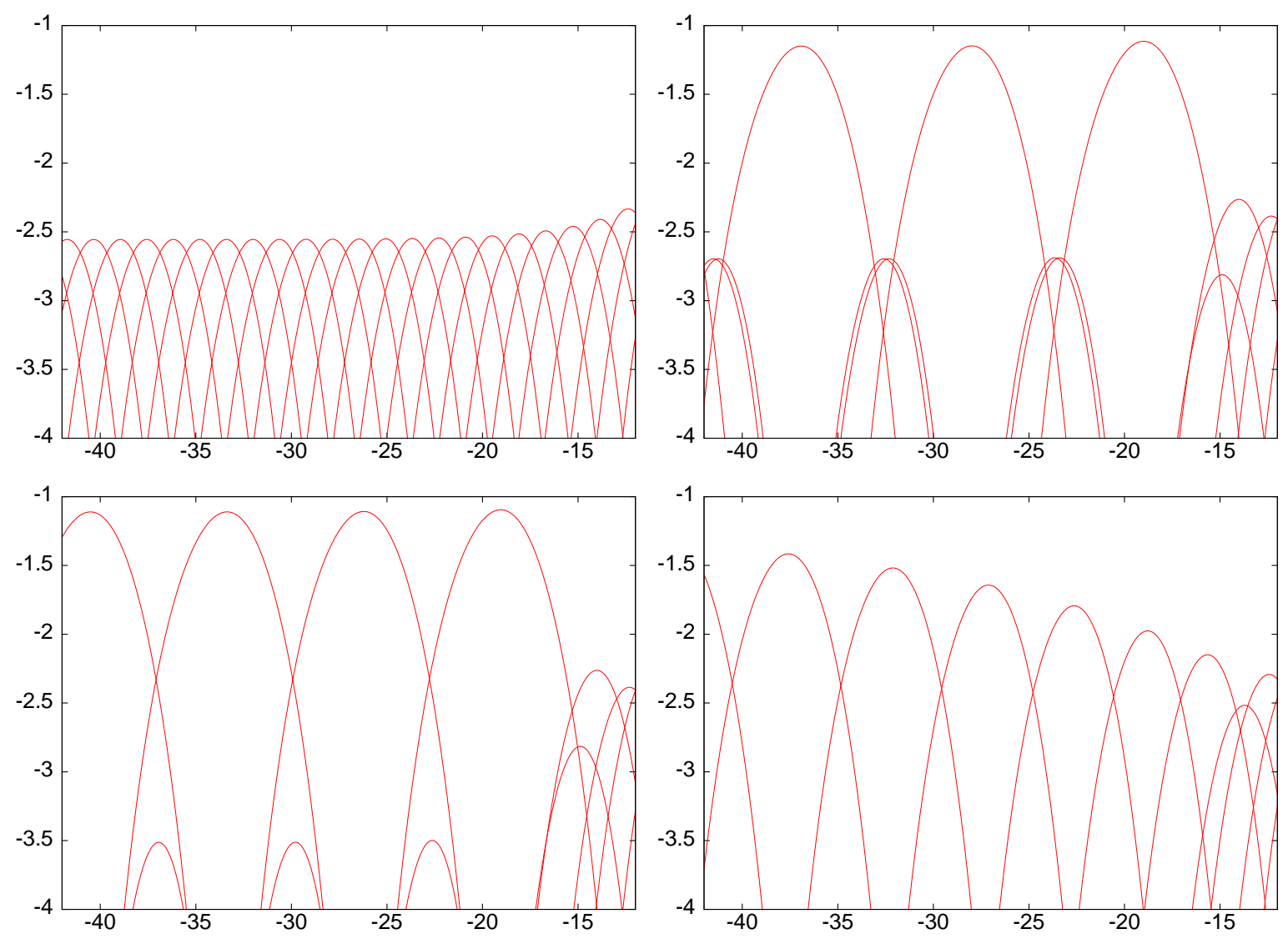

Figure 14: We represent the values of $\sqrt{\nu} \log \left(C_{m_{1}, m_{2}}\right)$ for the approximants $m_{1} / m_{2}$ that contribute to $\Delta F_{1}^{\{1\}} / \epsilon$ within the rang of $\nu$ in the plots $\left(\log _{2}(\nu)\right.$ ranges in the $x$-axis). Top left $($ Case 0$): \gamma=(\sqrt{5}-1) / 2$. Top right (Case 1): $\gamma=(55(1+b)+34) /(89(1+b)+55)$, with $b=(\sqrt{122}-10) / 11$, Bottom left (Case 2): $\gamma=(55(1+b)+34) /(89(1+b)+55)$, with $b=(\sqrt{140}-10) / 20$. Bottom right (Case 3$): \gamma=[0 ; 10 \times 1,2,3,4,5,6,7,8,9,10, \ldots]$. The same windows have been used in all plots for comparison.

Lemma 7.1. The distance between the $n$-th order approximant and $\gamma$, for arbitrary $\gamma \in \mathbb{R} \backslash \mathbb{Q}$, satisfies

$$
\left(D_{n}\left|D_{n} \gamma-N_{n}\right|\right)^{-1}=\left[q_{n+1} ; q_{n+2}, \ldots\right]+\left[0 ; q_{n}, q_{n-1}, \ldots, q_{1}\right] .
$$

Proof. From properties (ii), (iii) and (iv) one has

$$
\left|\gamma-\frac{N_{n}}{D_{n}}\right|=\frac{\beta_{n}}{D_{n}^{2}\left(1+\beta_{n} \frac{D_{n-1}}{D_{n}}\right)}=\frac{\beta_{n}}{D_{n}^{2}\left(1+\beta_{n}\left[0 ; q_{n}, q_{n+1}, \ldots, q_{1}\right]\right)} .
$$

This implies the result.

It is known that $\gamma \in \mathbb{R}$ is a quadratic irrational number if, and only if, its CFE is eventually periodic.

Lemma 7.2. Let $\gamma$ be a quadratic irrational number with eventually p-periodic CFE. Let $c_{s, n}=$ $c_{s, N_{n} / D_{n}}=\left(N_{n}\left|D_{n} \gamma-N_{n}\right|\right)^{-1}$. Then, the sequence of constants $\left\{c_{s, n}\right\}_{n \geq 1}$ is asymptotically p-periodic (as $n \rightarrow \infty)$. 
Proof. The statement follows from the relation

$$
\left(D_{n}^{2}\left|\gamma-\frac{N_{n}}{D_{n}}\right|\right)^{-1}=\frac{N_{n}}{D_{n}} c_{s, n},
$$

which, using the previous Lemma 7.1, implies that

$$
c_{s, n} \approx \frac{q_{+, n}+1 / q_{-, n}}{\gamma}\left(1+\mathcal{O}\left(D_{n}^{-2}\right)\right) .
$$

If $\gamma$ is a quadratic irrational number then, taking $n$ large enough, the sequence of quotients of $q_{+, n}$ is periodic and the one of $q_{-, n}$ tends to be periodic, that is, its quotients are periodic except maybe some final ones that have small influence on the value of $q_{-, n}$ if $n$ is large. This implies that $c_{s, n}$ tend to be periodic with respect to $n$ with the same period as the CFE of $\gamma$.

In particular, for the values of $\gamma$ referred as Cases 0, 1, and 2 in Section 7.6 one has:

Case $0: c_{s, n} \rightarrow 3+\gamma \approx 3.61803398$ as $n \rightarrow \infty$.

Case 1: $\left\{c_{s, n}\right\}_{n}$ tend to be 3-periodic. One has

$$
c_{s, n} \rightarrow 17.871271 \ldots, \quad c_{s, n+1}=c_{s, n+2} \rightarrow 3.249322 \ldots \text { for } n=2(\bmod 3) .
$$

Case 2: $\left\{c_{s, n}\right\}_{n}$ tend to be 2-periodic. One has,

$$
c_{s, n} \rightarrow a \approx 1.91442978 \text { for } n \text { even, and } \quad c_{s, n} \rightarrow 10 a \text { for } n \text { odd. }
$$

Then, for the $n$-th approximant of $\gamma$, say $N_{n} / D_{n}$, the corresponding maxima shown in Fig. 14]are approximated by $\Psi_{M} / \sqrt{c_{s, n}}$, being $\Psi_{M} \approx-4.860298$, and they are located at $\nu \approx L_{M} /\left(N_{n}^{2} c_{s, n}\right)$, where $L_{M} \approx 0.26236$. For example, in Case 2 the 4 -th visible maximum (from right to left) shown in the bottom left panel of Fig. 14 is related to $N=1034$ and corresponds to $c_{s, n} \rightarrow 10 a$. Accordingly its value is $\approx-1.1108186876015$ and it is $\operatorname{located}_{\text {at }} \log _{2}(\nu) \approx-26.2172640940432$ in agreement with what is shown in the figure.

\subsubsection{Diophantine properties of frequencies with unbounded CFE}

In Case 3 the frequency $\gamma$ has an unbounded CFE and $c_{s, n}$ tend to infinity as $n \rightarrow \infty$.

On the other hand, for $\gamma=e-2=[0 ; 1,2,1,1,4,1,1,6,1,1,8, \ldots]$ different behaviours of the constants $c_{s, n}$ are mixed. The sequence of best approximants $N_{n} / D_{n}$ of $\gamma=e-2$ is

$$
1 / 1,2 / 3,3 / 4,5 / 7,23 / 32,28 / 39,51 / 71,334 / 465,385 / 536,719 / 1001, \ldots
$$

The values $c_{s, n}$ associated to the approximant $N_{n} / D_{n}$ are such that the subsequence $\left\{c_{s, 3 m+1}\right\}_{m \geq 0}$ tends to $\infty$ linearly with slope $2 /(3 \gamma)$. The other two subsequences of $c_{s, n}$ are bounded, being $c_{s, 3 m}<c_{s, 3 m+2}$ for all $m \geq 1$, and they both tend to $2 / \gamma$. This explains the bumps observed in Fig. 12 ,

We give further details on the Diophantine properties of the previous unbounded CFE cases. For concreteness, we consider $\gamma_{1}=[0 ; 1,2,3,4,5,6,7,8, \ldots] \approx 0.69777465796400798200679$ and $\gamma_{2}=e-2=[0 ; 1,2,1,1,4,1,1,6,1,1,8, \ldots]$. As usual, to get Diophantine approximations the idea is to look for a function $\phi\left(D_{n}\right)$ such that $\phi\left(D_{n}\right)\left|D_{n} \gamma-N_{n}\right|$ is bounded from below. From the identity $c_{s, n}=\left(N_{n}\left|D_{n} \gamma-N_{n}\right|\right)^{-1}$ we can take $\phi\left(D_{n}\right)=N_{n} c_{s, n} \approx D_{n} \gamma c_{s, n}$, and we note that the constants $c_{s, n}$ can be approximated from the quotients $q_{n}$ of $\gamma$ using (40). 
Lemma 7.3. Let $\gamma_{1}=[0 ; 1,2,3,4,5,6,7,8, \ldots]$. There exists a constant $c>0$ such that ${ }^{3}$

$$
\left|q \gamma_{1}-p\right| \geq \frac{c}{\phi(q)}, \quad \phi(q)=q \log (q) / \log (\log (q))
$$

for all $p, q \in \mathbb{Z}$ with $q \geq 3$.

Proof. Since $q_{n}=n$ one has $q_{+, n}=(n+1)\left(1+\mathcal{O}\left(n^{-2}\right)\right), q_{-, n}=n\left(1+\mathcal{O}\left(n^{-2}\right)\right)$, and from (40) it follows that $c_{s, n} \gamma_{1} \approx(n+1)\left(1+\mathcal{O}\left(n^{-2}\right)\right)$. To obtain an explicit formula for $\phi(n)$ one has to relate $c_{s, n}$ with $D_{n}$. Note that $D_{n}=D_{n-1} q_{-, n}$ and then $D_{n}$ equals $n$ ! times a finite product of terms that are convergent when $n \rightarrow \infty$ (because $\sum_{n \geq 1} n^{-2}=\pi^{2} / 6$ ). Stirling's approximation provides the relation

$$
\log \left(D_{n}\right)=n \log n(1+\mathcal{O}(1 / \log (n)))
$$

which can be solved by Newton iteration (note that the Newton-Kantorovich theorem guarantees that the iteration starting with $n_{0}=\log \left(D_{n}\right) / \log \left(\log \left(D_{n}\right)\right)$ converges provided $D_{n}$ is large enough) to obtain

$$
n=\frac{\log \left(D_{n}\right)}{\log \left(\log \left(D_{n}\right)\right)}\left(1+\mathcal{O}\left(\frac{\log \left(\log \left(\log \left(D_{n}\right)\right)\right)}{\log \left(\log \left(D_{n}\right)\right)}\right)\right) .
$$

We conclude that $\phi\left(D_{n}\right)=D_{n} \log \left(D_{n}\right) / \log \left(\log \left(D_{n}\right)\right)$ ensures a positive lower bound of the scaled difference $\phi\left(D_{n}\right)\left|D_{n} \gamma_{1}-N_{n}\right|$. If $D_{n} \leq q<D_{n+1}$ then $\left|q-\gamma_{1} p\right| \geq\left|D_{n} \gamma_{1}-N_{n}\right| \geq c / \phi\left(D_{n}\right) \geq$ $c / \phi(q)$. Changing the constant $c$ we extend the inequality for $q \geq 3$.

Remark 7.2. Numerically we observe that $D_{n} / n ! \rightarrow 2.2796$ as $n \rightarrow \infty$. Let $\Pi_{n}=\phi\left(D_{n}\right)\left|D_{n} \gamma-N_{n}\right|$. The sequence $\left\{\Pi_{n}\right\}_{n>1}$ (for $n=1$ it is not defined!) reaches a minimum values for $n=6$ (that is when evaluated on the approximant $N_{6} / D_{6}=972 / 1393$ for which on has $\Pi_{6} \approx 0.50201173$ ) while uniformly increases for $n>6$. For $n=1000$ one has $\Pi_{1000} \approx 0.68014970$ and $\Pi_{n} \approx 0.758203198$ for $n=10^{5}$. The function $g(n)=\left|D_{n} \gamma_{1}-N_{n}\right| n D_{n}$ is such that $g(1)=1-\gamma_{1}$, it is monotonically increasing and it tends to one as $1-\mathcal{O}(1 / n)$ when $n \rightarrow \infty$ as expected.

We proceed similarly for $\gamma=\gamma_{2}=e-2$. The following lemma asserts that $\gamma_{2}$ has similar Diophantine properties to the ones described in Lemma 7.3 for $\gamma_{1}$.

Lemma 7.4. There exists a constant $c>0$ such that

$$
\left|q \gamma_{2}-p\right| \geq c / \phi(q), \quad \phi(q)=q \log (q) / \log (\log (q))
$$

for all $p, q \in \mathbb{Z}$ with $q \geq 3$.

Proof. We have $q_{n}=2(n+1) / 3$ if $n=2(\bmod 3)$ and $q_{n}=1$ otherwise. We consider $k=3 j+2$ below.

One has $q_{+, 3 j+1}=\left[q_{3 j+2} ; 1,1, q_{+, 3 j+4}\right]$ with $q_{3 j+2}=2(j+1)$ and $q_{+, 3 j+4}=\mathcal{O}(j)$, which implies that $q_{+, 3 j+1}=(2(j+1)+1 / 2)\left(1+\mathcal{O}\left(j^{-2}\right)\right)=(2(k+1) / 3+1 / 2)\left(1+\mathcal{O}\left(k^{-2}\right)\right)$. From the relation $q_{+, 3 j}=q_{3 j+1}+1 / q_{+, 3 j+1}$ it follows that $q_{+, 3 j}=(1+3 /(2 k))\left(1+\mathcal{O}\left(k^{-2}\right)\right)$. Using that $q_{+, 3 j+2}=\left[1 ; 1, q_{+, 3 j+4}\right]$ where $q_{+, 3 j+4}=(2(j+2)+1 / 2)\left(1+\mathcal{O}\left(j^{-2}\right)\right)$ one obtains $q_{+, 3 j+2}=$ $(2-3 /(2 k))\left(1+\mathcal{O}\left(k^{-2}\right)\right)$.

Moreover, since $q_{-, 3 j+2}=\left[2(j+1) ; 1,1, q_{-, 3 j-1}\right]$ and $q_{-, 3 j-1}=\mathcal{O}(j)$, one gets $q_{-, 3 j+2}=(2(k+$ $1) / 3+1 / 2)\left(1+\mathcal{O}\left(k^{-2}\right)\right)$. From $q_{-, 3 j+1}=\left[q_{3 j+1} ; q_{3 j}, q_{-, 3 j-1}\right]$, using that $q_{-, 3 j-1}=2 j\left(1+\mathcal{O}\left(j^{-1}\right)\right)$,

\footnotetext{
${ }^{3}$ Note that $\phi(q)<q^{\tau}$ for any $\tau>1$ if $q$ is large enough. Equivalently, $\gamma_{1}$ satisfies the Diophantine condition $\left|\gamma_{1}-p / q\right| \geq c / q^{\tau}$ for any $\tau=2+\epsilon, \epsilon>0$ and a suitable constant $c=c(\epsilon)>0$.
} 
it follows that $q_{-, 3 j+1}=(2-3 /(2 k))\left(1+\mathcal{O}\left(k^{-2}\right)\right)$. Since $q_{-, 3 j}=\left[q_{3 j} ; q_{-, 3 j-1}\right]$ one obtains $q_{-, 3 j}=(1+3 /(2 k))\left(1+\mathcal{O}\left(k^{-2}\right)\right)$.

Summarizing, for $k=2(\bmod 3)$, we obtain

$$
\begin{array}{lll}
q_{+, k-2} \approx 1+\frac{3}{2 k}, & q_{+, k-1} \approx \frac{2(k+1)}{3}+\frac{1}{2}, & q_{+, k} \approx 2-\frac{3}{2 k}, \\
q_{-, k-2} \approx 1+\frac{3}{2 k}, & q_{-, k-1} \approx 2-\frac{3}{2 k}, & q_{-, k} \approx \frac{2(k+1)}{3}+\frac{1}{2},
\end{array}
$$

with a relative error $\mathcal{O}\left(k^{-2}\right)$ in all cases. Using (40) and the previous estimates we get $c_{s, k-2} \gamma_{2} \approx$ $2, c_{s, k-1} \gamma_{2} \approx 2(k+1) / 3+1$ and $c_{s, k} \gamma_{2} \approx 2$. We conclude that $c_{s, n}, n \geq 1$ is, at most, $\mathcal{O}(n)$.

Next, we look to the denominators $D_{n}$. We use the properties listed in the items before Lemma 17.1. The recurrence $D_{n}=q_{n} D_{n-1}+D_{n-2}$, implies that $D_{3 j+2}=2(j+1)\left(D_{3 j}+\right.$ $\left.D_{3 j-1}\right)+D_{3 j}$ and, using the identity $D_{3 j}=D_{3 j-1} q_{-, 3 j}$, it simplifies to

$$
D_{3 j+2}=\left(2(j+1)\left(1+q_{-, 3 j}\right)+q_{-, 3 j}\right) D_{3 j-1} .
$$

Since $q_{-, 3 j}=\left[q_{3 j} ; q_{-, 3 j-1}\right]=(1+1 /(2 j))\left(1+\mathcal{O}\left(j^{-2}\right)\right)$, one obtains

$$
D_{3 j+2}=4\left(j+\frac{3}{2}\right) D_{3 j-1}\left(1+\mathcal{O}\left(j^{-2}\right)\right)=\frac{4 k+10}{3} D_{3 j-1}\left(1+\mathcal{O}\left(k^{-2}\right)\right) .
$$

From this recurrence we obtain $D_{3 j+2} \approx 4^{j} \Gamma(j+5 / 2)$ or, equivalently,

$$
D_{n} \approx 4^{n / 3} \Gamma(n / 3+11 / 6), \quad \text { for } n \text { such that } n=2(\bmod 3) .
$$

Then, Stirling's approximation gives

$$
3 \log D_{n} \approx n \log n-a n+4 \log n, \quad a=1+\log 3 / 4 .
$$

As in Lemma 7.3, we take $n_{0}=3 \log \left(D_{n}\right) / \log \left(\log \left(D_{n}\right)\right)$ and solve this relation by (Newton) iteration (the convergence follows from the Newton-Kantorovich theorem). We obtain

$$
n=\frac{3 \log \left(D_{n}\right)}{\log \left(\log \left(D_{n}\right)\right)}\left(1+\mathcal{O}\left(\frac{\log \left(\log \left(\log \left(D_{n}\right)\right)\right)}{\log \left(\log \left(D_{n}\right)\right)}\right)\right) .
$$

Now the proof finishes as the proof of Lemma 7.3. Since $c_{s, n}=\mathcal{O}(n)$ then one takes $\phi(q)=$ $q \log (q) / \log (\log (q))$ to have values of $\left|q \gamma_{2}-p\right| \phi(q)$ bounded from below.

We show in Fig. 15]left the values of $\xi_{n}=\left|D_{n} \gamma_{2}-N_{n}\right| \phi\left(D_{n}\right)$ as a function of $n$. In the right plot we only show the local minima of $\xi_{n}$ (i.e. those corresponding to $\left.n=1(\bmod 3)\right)$. According to the theoretical predictions, the minimum values tend to a constant.

Remark 7.3. We have seen that the frequencies $\gamma_{1}$ and $\gamma_{2}$ satisfy a condition of the form $|q \gamma-p| \geq$ $c \log (\log (q)) /(q \log (q)), q \geq 3$. We remark that the set of irrational numbers $\gamma$ satisfying such a type of condition for some $c>0$ has zero measure. By contrast, the set of irrational numbers $\gamma$ satisfying a condition of the form $|q \gamma-p| \geq c / \phi(q)$ for $q \geq q_{0}$ for some $c>0$ and such that $1 / \phi(q)$ is integrable in the range $\left[q_{0}, \infty\right)$, has total measure. We refer to [14] for further details on measure aspects of CFE. As examples we can consider numbers of the form $\gamma=\left[0 ; 1^{m}, 2^{m}, 3^{m}, 4^{m}, 5^{m}, \ldots\right]$ for $1<m \in \mathbb{Z}_{+}$. They satisfy $|q \gamma-p| \geq c(\log (\log (q)))^{m} /\left(q(\log (q))^{m}\right)$ for $q \geq 3$ and a positive constant $c$.

Remark 7.4. It follows from the reasoning in Remark 5.1 that if $\gamma$ satisfies a Diophantine condition of the form $|q \gamma-p| \geq c|q|^{-\tau}, \tau \geq 1, c>0$, then the exponentially small part of the splitting is expected to have an exponent of the form $-C / \nu^{1 /(\tau+1)}$ with $C>0$. A similar reasoning shows that if $\gamma$ satisfies 

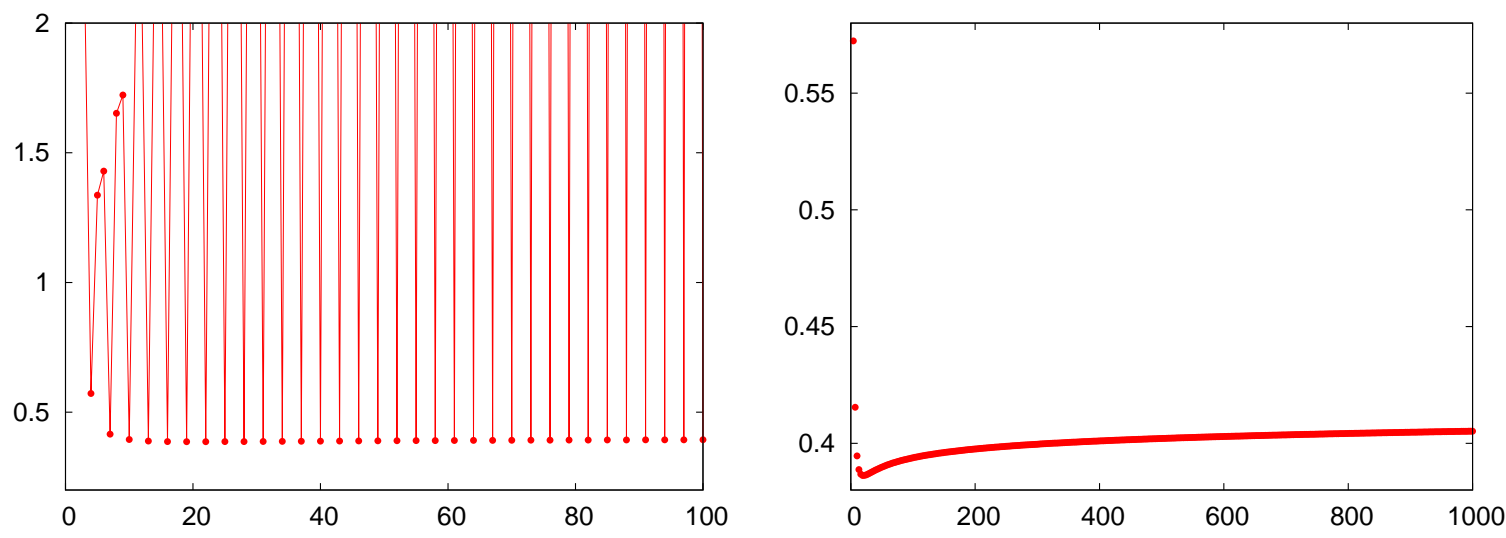

Figure 15: Left: We represent $\xi_{n}=\left|D_{n} \gamma_{2}-N_{n}\right| \phi\left(D_{n}\right)$ as a function of $n$. In the right plot we focus on the behaviour of the minima (up to $n=1000$ ).

a condition of the form $|q \gamma-p| \geq c \log (\log (q)) /(q \log (q)), q \geq 3, c>0$, as the ones considered in this section, then the exponent of the exponentially small part becomes

$$
\left(\frac{-C \sqrt{\log (\log (\sqrt{1 / \nu})})}{\sqrt{\nu \log (\sqrt{1 / \nu})}}\right)(1+o(1)), \quad C>0,
$$

where the $o(1)$ terms are bounded by $\log (\log (1 / \nu)) / \log (1 / \nu)$. Consequently, if one represents $\log \left(\left|\Delta F_{1}^{1}\right| / \epsilon\right)$ multiplied by $\sqrt{\nu \log (1 / \nu) / \log (\log (1 / \nu))}$ (instead of by $\sqrt{\nu}$ as a function of $\log _{2}(\nu)$ as we did in Fig. 12 and in Case 3 of Fig. (14), then the maxima tend to a constant value. In the multiplying factor we have neglected $\log (2)$ in front of $\log (\sqrt{1 / \nu})$.

\section{Conclusions and future work}

In this work we have investigated the asymptotic properties of the splitting of the invariant manifolds emanating from a complex-saddle fixed point of a 2-dof Hamiltonian system $H_{0}(\mathbf{x}, \mathbf{y})$ undergoing a Hamiltonian-Hopf bifurcation (at $\nu=0$ ) when acting a periodic forcing on the system. We have obtained detailed information of the exponentially small behaviour, describing the changes of dominant harmonic as $\nu \rightarrow 0$. As has been discussed through the paper, for the concrete example considered, when using Poincaré-Melnikov method it remains to bound the effect of

- the terms in the first order Melnikov approximation not related to best approximants and,

- the non-dominant terms in the splitting function, bounding the effect of higher order Melnikov approximations.

In any case, the detailed description presented in this paper takes advantage of the concrete properties of the explicit periodic perturbation $\epsilon H_{1}(\mathbf{x}, \mathbf{y}, t)$ considered. In this sense, an interesting topic for future works would be to consider other perturbations $H_{1}(\mathbf{x}, \mathbf{y}, t)$, for example:

- perturbations having a finite number of harmonics (then higher order Melnikov analysis could be required to analyse the changes of dominant harmonic).

- perturbations leading to a periodic orbit from the fixed point of the system. 
As a consequence of the splitting of the invariant manifolds, in a neighbourhood of the stable/unstable invariant manifolds there is a region where rich dynamics appears. A desirable tool to investigate this dynamics would be a suitable return map adapted to this problem. Such a return map depends on two key ingredients: the return time to a suitable Poincaré section and the splitting function. This is an interesting problem, motivated by the slow diffusive expected properties (see Appendix (D), that we postpone to study in future works. Concretely, it involves:

- to construct a 4D separatrix map adapted to this problem. As said, this requires not only the splitting function (see Section 5 ) but also the passage time close to the complex-saddle point, and

- to provide a description of the geometry of the phase space (resonance web) and analyse the diffusive properties of the model. Note, however, that to observe the asymptotic behaviour requires very small values of $\nu$, outside the range of interest of any physical application.

Finally, we also note that the case considered is somehow an intermediate case between the 2-dof Hamiltonian case (in which the splitting behaves as the one of a periodic perturbation of an integrable system) and the splitting of the separatrices for a family of $4 \mathrm{D}$ symplectic maps undergoing a Hamiltonian-Hopf bifurcation (in which the perturbation is not explicit). Then, a natural continuation of this work would be to consider the analogous Hamiltonian-Hopf bifurcation for $4 \mathrm{D}$ symplectic maps and to study the splitting of the invariant manifolds and the consequences in the diffusion properties.

\section{A Autonomous perturbation of the system}

In this appendix we study the effect of an autonomous entire perturbation of $H_{0}$ in (11) of the form

$$
H(\mathbf{x}, \mathbf{y})=H_{0}(\mathbf{x}, \mathbf{y})+\epsilon H_{1}(\mathbf{x}, \mathbf{y}),
$$

where $H_{1}(\mathbf{x}, \mathbf{y})=\sum_{k_{1}, k_{2}, l_{1}, l_{2}} x_{1}^{k_{1}} x_{2}^{k_{2}} y_{1}^{l_{1}} y_{2}^{l_{2}}$, and where the sum is considered over a finite number of indices $k_{1}, k_{2}, l_{1}, l_{2} \in \mathbb{N}$. We recall that $H_{0}$ depends on $\nu$. We assume that the perturbation keeps zero as an equilibrium point. In this case, $H(\mathbf{x}, \mathbf{y})$ is a first integral and both $W^{u / s}(\mathbf{0})$ lie on $H=0$. Consequently, the splitting can be measured by the variation of $F_{1}=\Gamma_{1}(\mathbf{x}, \mathbf{y})=$ $x_{1} y_{2}-x_{2} y_{1}$ which is a first integral of the unperturbed system.

Let $\mathbf{p}_{\mathbf{0}}$ be a point in the 2-dimensional homoclinic connection of the unperturbed system given by (14) with $t=0$ and angle $\psi_{0}$. We identify $\mathbf{p}_{0}$ with $\psi_{0}$. Denote by $\varphi\left(t, \mathbf{p}_{\mathbf{0}}\right)$ the unperturbed solution starting at $\mathbf{p}_{\mathbf{0}}$. The first-order Melnikov function to measure the variation of $F_{1}$ is given by

$$
M_{1}\left(\psi_{0}\right)=\int_{-\infty}^{\infty}\left\{\Gamma_{1}\left(\varphi\left(t, \mathbf{p}_{\mathbf{0}}\right)\right), H_{1}\left(\varphi\left(t, \mathbf{p}_{\mathbf{0}}\right)\right)\right\} d t=\int_{-\infty}^{\infty}\left(D \Gamma_{1} \cdot \mathbb{J} D H_{1}^{\top}\right)_{\mid \varphi\left(t, \mathbf{p}_{\mathbf{0}}\right)} d t,
$$

see 22 for details of the Melnikov method in this context. By linearity it is enough to consider separately the effect of every individual monomial, i.e. $H_{1}\left(x_{1}, x_{2}, y_{1}, y_{2}\right)=x_{1}^{k_{1}} x_{2}^{k_{2}} y_{1}^{l_{1}} y_{2}^{l_{2}}$. Let $r=k_{1}+k_{2}+l_{1}+l_{2}$ be the degree of the monomial. One has

$$
M_{1}\left(\psi_{0}\right)=\int_{-\infty}^{\infty}\left[m \cos ^{m-1}(\psi) \sin ^{n+1}(\psi)-n \cos ^{m+1}(\psi) \sin ^{n-1}(\psi)\right] R(t) d t,
$$

where $R(t)=R_{1}(t)^{k} R_{2}(t)^{l}, k=k_{1}+k_{2}, l=l_{1}+l_{2}, m=k_{1}+l_{1}, n=k_{2}+l_{2}$ and $\psi=\psi(t)=t+\psi_{0}$, $\psi_{0} \in \mathbb{R}$, and $R_{1}(t), R_{2}(t)$ as given in Section 3.1 
We consider first the case $r$ odd. For the expansions below, we introduce

$$
C(\hat{n}, \hat{m}):=\sum_{s=0}^{\hat{n}}(-1)^{s}\left(\begin{array}{c}
\hat{n} \\
s
\end{array}\right)\left(\begin{array}{c}
\hat{m} \\
(r+1) / 2-s
\end{array}\right)=(-1)^{\hat{n}} \sum_{s=0}^{\hat{n}}(-1)^{s}\left(\begin{array}{c}
\hat{n} \\
s
\end{array}\right)\left(\begin{array}{c}
\hat{m} \\
(r-1) / 2-s
\end{array}\right),
$$

where $r=\hat{m}+\hat{n}$ and where the equality follows from Pascal's rule. Here, if $b>a$, we assume $\left(\begin{array}{l}a \\ b\end{array}\right)=0$. Consequently, if one considers just the contribution of the dominant first-order harmonic one has

$$
M_{1}\left(\psi_{0}\right) \approx \frac{m C_{1}+n C_{2}}{2^{r-1}} \int_{-\infty}^{\infty} \frac{\left(e^{i \psi}-(-1)^{n} e^{-i \psi}\right)}{2 i^{n-1}} R(t) d t
$$

where $C_{1}=C(n+1, m-1)$ and $C_{2}=C(n-1, m+1)$. We note that $m C_{1}+n C_{2} \neq 0$. The evaluation of the previous integral reduces to a linear combination (with coefficients depending on $\psi_{0}$ ) of integrals of the form

$$
\int_{-\infty}^{\infty} \sin (t) \sinh ^{k}(\nu t) \cosh ^{-(2 k+l)}(\nu t) d t \quad \text { and } \quad \int_{-\infty}^{\infty} \cos (t) \sinh ^{k}(\nu t) \cosh ^{-(2 k+l)}(\nu t) d t .
$$

One of the two previous integrals vanishes (depending on the parity of $k$ ). The other can be evaluated by residues and one obtains

$$
M_{1}\left(\psi_{0}\right) \approx A \frac{e^{-\frac{\pi}{2 \nu}}}{\nu^{r+k}}
$$

where $A=A\left(\psi_{0}\right)$ is a suitable constant depending on $C_{1}, C_{2}$ and either $\sin \left(\psi_{0}\right)$ or $\cos \left(\psi_{0}\right)$.

The case $r$ even can be handled similarly but, in this case, the dominant harmonic is the second order one. One obtains

$$
M_{1}\left(\psi_{0}\right) \approx \tilde{A} \frac{e^{-\frac{\pi}{\nu}}}{\nu^{r+k}}
$$

for a suitable constant $\tilde{A}=\tilde{A}\left(\psi_{0}\right)$. Note that for a fixed value of $\nu$ the splitting size is expected to be much smaller when $r$ is even than when it is odd.

The coefficient $A$ and $\tilde{A}$ above follow for a simple monomial but in the case of a polynomial one can have cancellations.

Remark A.1. If one uses $F_{2}=\Gamma_{2}-\Gamma_{3}+\Gamma_{3}^{2}$ instead of $F_{1}=\Gamma_{1}$ to measure the splitting then one obtains

$$
M_{2}\left(\psi_{0}\right)=\mathcal{O}\left(\frac{1}{\nu} M_{1}\left(\psi_{0}\right)\right)=\mathcal{O}\left(\frac{A}{\nu^{r+k+1}} e^{-\frac{\pi}{2 \nu}}\right) .
$$

In general the prefactor depends on the first integral we use.

Example. If $H(\mathbf{x}, \mathbf{y})=H_{0}(\mathbf{x}, \mathbf{y})+\epsilon y_{1}^{5}$ (i.e. $\left.r=5, k=0, l=5, m=5, n=0\right)$ then

$$
M\left(\psi_{0}\right)=\int_{-\infty}^{+\infty} 5 \cos ^{4} \psi \sin \psi R_{2}(t)^{5} d t=\sum_{j=0}^{5} A_{j, 0,5}\left(\psi_{0}\right) I_{0,5}(j)
$$

where

$$
I_{0,5}(j)=\int_{-\infty}^{+\infty} \sin ^{j} t \cos ^{5-j} t \frac{1}{\cosh ^{5}(\nu t)} d t
$$

and $A_{0,0,5}=s_{0}^{4} c_{0}$,

$$
A_{j, 0,5}\left(\psi_{0}\right)=(-1)^{j} 20 \sqrt{2}\left[\left(\begin{array}{l}
4 \\
j
\end{array}\right) s_{0}^{j-1} c_{0}^{j+1}+\left(\begin{array}{c}
4 \\
j-1
\end{array}\right) s_{0}^{5-j} c_{0}^{j}\right], \quad 1 \leq j \leq 4,
$$



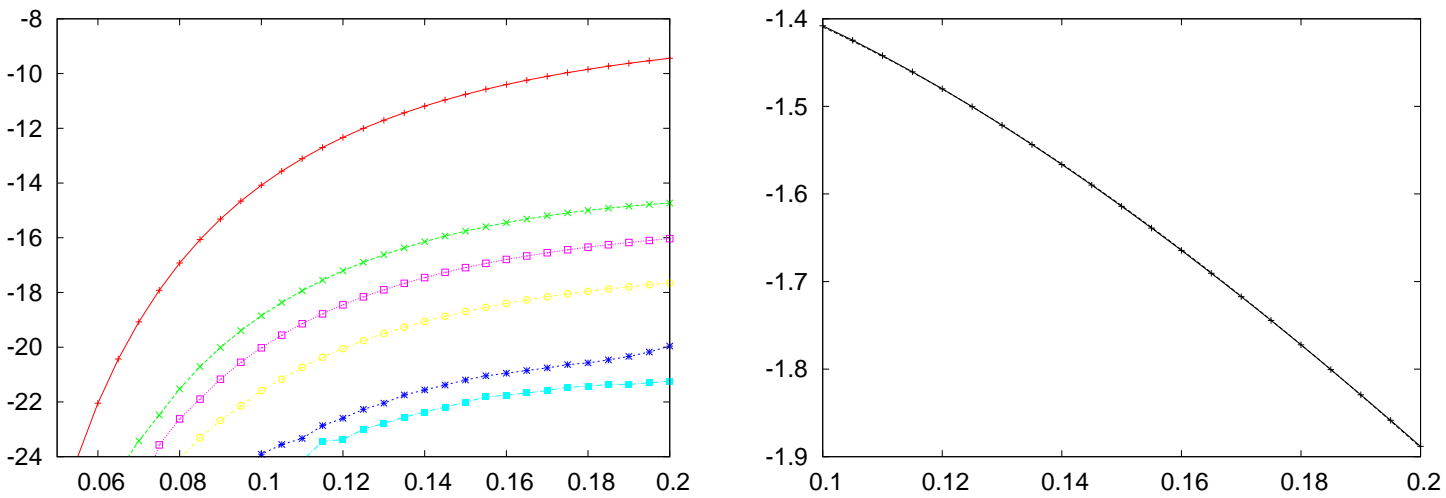

Figure 16: Left: We represent the amplitude $A_{i}, i=1, \ldots, 6$, of the main 6 harmonics of the difference $\Delta \Gamma=\Gamma_{1}\left(W^{u}\right)-\Gamma_{1}\left(W^{s}\right)$ with respect to $\nu$. For $\nu=0.2$ the lines correspond, from top to bottom, to the $i$-th harmonic ordered as follows: $i=1,2,4,6,3,5$. Right: A numerical fit of $\nu \log \left(A_{1}\right)$ as a function of $\nu$ by a function $g(x)=a x+b x \log (x)+c+d x^{2}$. One obtains $c \approx-1.58898$ and $b \approx-5.51$ if fitting in the interval shown. If one fixes $c=\pi / 2$ then $b=-5.256$.

and $A_{5,0,5}=c_{0}^{4} s_{0}$, being $s_{0}=\sin \left(\psi_{0}\right)$ and $c_{0}=\cos \left(\psi_{0}\right)$. It is easier to look directly for the contribution of the first harmonic, we know that it is non-zero because $r=5$ is odd. One has $\cos ^{4} \psi \sin \psi=(\sin \psi) / 8+$ hoh, where hoh denotes the terms with higher order harmonics. Writting $\sin (\psi)=\sin t \cos \psi_{0}+\cos t \sin \psi_{0}$, one sees that only the term in $\cos t \sin \psi_{0} \cosh ^{-5}(\nu t)$ contributes to the Melnikov integral, which is reduced to

$$
\frac{5}{32 \sqrt{2}} \sin \left(\psi_{0}\right) \int_{-\infty}^{+\infty} \frac{\cos t}{\cosh ^{5}(\nu t)} d t=\frac{5 \pi \sin \psi_{0}}{384 \sqrt{2}} \frac{1}{\nu^{5}} e^{-\frac{\pi}{2 \nu}}\left(1+O\left(\nu^{2}\right)\right)
$$

We check the results by a direct numerical computation of the splitting between $W^{u}$ and $W^{s}$ in terms of $F_{1}=\Gamma_{1}$. We consider $\epsilon=10^{-4}$. The invariant manifolds of the unperturbed system $(\epsilon=0)$ intersect the Poincaré section $\Sigma=\max \left\{y_{1}^{2}+y_{2}^{2}\right\}$ in the curve $x_{1}=0, y_{1}^{2}+y_{2}^{2}=2$. These manifolds are contained in the level set $F_{1}^{-1}(0)$ (and $F_{2}^{-1}(0)$ ). For $\epsilon \neq 0$, this is no longer true because of the splitting. For different values of $\nu$, we propagate $N=100$ initial conditions on the linear approximation at the origin of the invariant manifold $W^{u}$ (using quadruple precision) up to $\Sigma$ and we evaluate $\Gamma_{1}$ on the reached points. We repeat the process for $N=100$ points on $W^{s}$. Concretely, we select the initial conditions as follows: given a mesh of equidistributed angles $\psi_{0} \in[0,2 \pi)$ and fixed $R_{2}=10^{-12}$, we set $R_{1}=R_{2}\left(1-R_{2}^{2} / 2\right)$ and we take $y_{1}^{u}=$ $R_{2} \cos \left(\psi_{0}\right), y_{2}^{u}=R_{2} \sin \left(\psi_{0}\right), x_{1}^{u}=R_{1} \cos \left(\psi_{0}\right), x_{2}^{u}=R_{1} \sin \left(\psi_{0}\right)$ as initial condition on $W^{u}$, and $y_{1}^{s}=y_{1}^{u}, y_{2}^{s}=y_{2}^{u}, x_{1}^{s}=-x_{1}^{u}, x_{2}^{s}=-x_{2}^{u}$, as initial condition on $W^{s}$.

Let $\theta=\arctan \left(y_{2} / y_{1}\right)$. To look for the behaviour of the splitting with respect to $\nu$, we fit $\Gamma_{1}\left(W^{u}\right)$ (resp. $\Gamma_{1}\left(W^{s}\right)$ ), evaluated on the invariant manifolds obtained by numerical integration in $\Sigma$, by a function of the form $\Gamma_{1}(\theta)=\sum_{k=1}^{6} a_{k}^{u}(\nu) e^{i k \theta}$ (resp. with $a_{k}^{s}$ for $W^{s}$ ). Then we compute the splitting as the real part of the difference $\Delta \Gamma=\Gamma_{1}\left(W^{u}\right)-\Gamma_{1}\left(W^{s}\right)=\sum_{k=1}^{6} a_{k}(\nu) e^{i k \theta}$, where $a_{k}=a_{k}^{u}-a_{k}^{s}$, to obtain the amplitude of the first harmonics of the splitting in terms of $\Gamma_{1}$. The results are shown in Fig. 16. A numerical fit shows quite a good agreement with the predicted formula (42). 


\section{B An example: a periodic perturbation of the Duffing equation}

We consider a simple example to illustrate that the sum of all terms in a series expansion can change the dominant exponent in the Melnikov function.

Our model is given by $H(x, y, t)=H_{0}(x, y)+\epsilon H_{1}(x, y, t)$ where

$$
H_{0}(x, y)=\frac{y^{2}}{2}-\frac{x^{2}}{2}+\frac{x^{4}}{4}
$$

is the so-called Duffing Hamiltonian, and

$$
H_{1}(x, y, t)=\frac{x^{2}}{d-x} \sin (\omega t)=f(x) \sin (\omega t),
$$

with $d>\sqrt{2}$. The unperturbed system $H_{0}(x, y)$ possesses a homoclinic orbit $\varphi(t)=(x(t), y(t))=$ $(\sqrt{2} / \cosh (t),-\sqrt{2} \tanh (t) / \cosh (t))$.

This is a regular perturbation problem and hence the splitting of the invariant manifolds for $\epsilon>0$ is expected to be $\mathcal{O}(\epsilon)$. We consider the Melnikov function $M(\alpha)$ to compute the first order in $\epsilon$ of this splitting. It is given by

$$
\begin{aligned}
M(\alpha) & =\int_{-\infty}^{+\infty}\left\{H_{0}, H_{1}\right\}(\varphi(t), t+\alpha) d t=-\int_{-\infty}^{+\infty} y \frac{d}{d x}\left(\frac{x^{2}}{d-x}\right) \sin (\omega t)(\varphi(t), t+\alpha) d t \\
& =\omega \cos (\omega \alpha) I_{c}-\omega \sin (\omega \alpha) I_{s},
\end{aligned}
$$

where

$$
I_{c}=\int_{-\infty}^{+\infty} \frac{x^{2}(t)}{d-x(t)} \cos (\omega t) d t, \quad I_{s}=\int_{-\infty}^{+\infty} \frac{x^{2}(t)}{d-x(t)} \sin (\omega t) d t .
$$

We have that $I_{s} \equiv 0$ because $x(t)$ is an even function of $t$.

Note that $f(x(t))$ is a $2 \pi \mathrm{i}$-periodic function in $t$. It has singularities at $\mathrm{i}(\pi / 2+k \pi), k \in \mathbb{Z}$, because it contains $\cosh (t)$. Also it has singularities at $\left( \pm s_{0}+2 k \pi\right) \mathrm{i}, k \in \mathbb{Z}$, where $s_{0}=$ $\arccos (\sqrt{2} / d) \in(0, \pi / 2)$, due to the denominator. We just note that the singularity i $s_{0}$ is closer to the real axis of time than $\mathrm{i} \pi / 2$.

Let us proceed in two different ways to evaluate $I_{c}$.

Evaluation by residues. One has

$$
I_{c}=\int_{-\infty}^{\infty} \frac{x^{2}(t)}{d-x(t)} e^{i \omega t} d t
$$

We integrate along the boundary of the rectangle of vertices $-R, R, R+2 \pi \mathrm{i},-R+2 \pi \mathrm{i}$, with $R>0$ and then we take the limit $R \rightarrow \infty$. Inside there are four singularities of the integrand $F(t)=e^{\mathrm{i} \omega t} x^{2} /(d-x)$ : i $s_{0}, \mathrm{i} \pi / 2, \mathrm{i} 3 \pi / 2$ and $\mathrm{i}\left(2 \pi-s_{0}\right)$. One gets

$$
I_{c}=2 \pi \mathrm{i} \frac{1}{1-e^{-2 \pi \omega}} \sum \operatorname{Res}(F, *),
$$

where $\sum \operatorname{Res}(F, *)$ denotes the sum of the residues at the four singularities. The residues are

$$
\begin{aligned}
\operatorname{Res}\left(F, \mathrm{i} s_{0}\right) & =-\mathrm{i} \frac{\sqrt{2} d}{\sqrt{d^{2}-2}} e^{-\omega s_{0}}, & \operatorname{Res}\left(F, \mathrm{i}\left(\pi-s_{0}\right)\right) & =\mathrm{i} \frac{\sqrt{2} d}{\sqrt{d^{2}-2}} e^{\omega\left(s_{0}-2 \pi\right)}, \\
\operatorname{Res}(F, \mathrm{i} \pi / 2) & =\mathrm{i} \sqrt{2} e^{-\omega \pi / 2}, & \operatorname{Res}(F, \mathrm{i} 3 \pi / 2) & =-\mathrm{i} \sqrt{2} e^{-3 \omega \pi / 2} .
\end{aligned}
$$


Note that under a fast frequency perturbation, for example if $\omega \sim \nu^{-1}$ with $|\nu|$ small, the integral $I_{c}$ is $\mathcal{O}\left(e^{-\omega s_{0}}\right)$ asymptotically when $\nu \rightarrow 0$.

Evaluation by series expansion of $f(x)$. We expand

$$
f(x)=\frac{x^{2}}{d-x}=\frac{x^{2}}{d} \sum_{j \geq 0}\left(\frac{x}{d}\right)^{j}
$$

and substitute this expansion into the Poincaré-Melnikov integral. The previous series converges uniformly and the series of the integrals also is convergent. One gets

$$
I_{c}=\sum_{j \geq 0} \frac{1}{d^{j+1}} \int_{-\infty}^{\infty} x^{j+2}(t) \cos (\omega t) d t=\sum_{j \geq 0} \frac{\sqrt{2}^{j+2}}{d^{j+1}} I_{j+2}=d \sum_{k \geq 2}\left(\frac{\sqrt{2}}{d}\right)^{k} I_{k},
$$

where

$$
I_{k}=\int_{-\infty}^{\infty} \frac{\cos (\omega t)}{\cosh ^{k}(t)} d t .
$$

Note that with the notation used in (23) one has $I_{n}=I_{1}(\omega, 1, n)$. Then the following recurrence holds for the integrals $I_{n}$

$$
I_{1}=\frac{\pi}{\cosh (\omega \pi / 2)}, \quad I_{2}=\frac{\omega \pi}{\sinh (\omega \pi / 2)}, \quad I_{n}=\frac{\omega^{2}+(n-2)^{2}}{(n-1)(n-2)} I_{n-2}, n \geq 3 .
$$

Each $I_{n}$ is related to the monomial $x^{n}$ of the series of $f$.

Let us provide an idea of the behaviour of the series for $\omega$ large. A lower bound can be obtained by neglecting the $(n-2)^{2}$ terms (compare to $\left.\omega^{2}\right)$ in the numerator of the recurrence of $I_{n}$. Letting aside constants, one can write $I_{1}=\exp (-\omega \pi / 2), I_{2}=\omega \exp (-\omega \pi / 2)$ and then one has $I_{n}=\omega^{n-1} \exp (-\omega \pi / 2)$. The sum in the previous expression of $I_{c}$ becomes (for simplicity we consider the sum starting at $k=0$, which adds relatively small contributions)

$$
\omega^{-1} \sum_{k \geq 0}\left(\frac{\sqrt{2} \omega}{d}\right)^{k} \exp (-\omega \pi / 2)=\omega^{-1} \exp (-\omega(\pi / 2-\sqrt{2} / d)) .
$$

As mentioned, this is a lower bound of the sum. If we compare the exponential part with the one obtained by residues, the relevant multiples of $-\omega$ in the exponents are $\pi / 2-\sqrt{2} / d$ and $s_{0}=\arccos (\sqrt{2} / d)$. They are quite close for large $d$, but differ in an important way when $d$ tends to $\sqrt{2}$.

To see the contribution of the largest term in the sum, let us look at a general case: given a large value $z$, how the largest term in the series for $\exp (z)$ compares with the sum. Obviously the largest term is the term $n=[z]$, where [ ] denotes the integer part. For simplicity we use $n=z$ and the relative contribution is $\left(z^{z} / \Gamma(z+1)\right) / \exp (z) \approx 1 / \sqrt{2 \pi z}$, using Stirling formula.

Summarizing, the largest term in the series can give some idea of the total contribution to the splitting, but only for large values of $d$.

Remark B.1. This appendix seems to contradict the ideas in this work since, for the system (11) considered, we claim that the dominant term of the series expansion provides the correct order (that is the correct exponent in the exponentially small part) of the splitting function. The explanation is a quasi-periodic effect: the contribution of the small divisors related to the two frequencies to the terms related to the dominant harmonics of the splitting function is of larger order than the contribution of the other terms of the expansion. Let us give further details. The dynamics along the homoclinic orbit of 
system (11) is slow. Scaling $T=\nu t$ it becomes of order 1 , as it is for the example of this appendix. The singularities closest to the real axis are $\pm \mathrm{i} \arccos (\sqrt{2} / d)$ and $\pm \mathrm{i} \log \left(c+\sqrt{c^{2}-1}\right)$. The angles playing a role are $\psi=T / \nu+\psi_{0}$ and $\theta=\gamma T / \nu+\theta_{0}$, hence fast angles. For $c=5$ and $d=7$, the closest singularities are located at $\pm \mathrm{i} \sigma_{d}, \sigma_{d} \approx 1.367365$ and at $\pm \mathrm{i} \sigma_{c}, \sigma_{c} \approx 2.292431$. Hence under a periodic perturbation one would expect an exponentially small splitting in $-\sigma_{d} / \nu$. However, under a quasi-periodic perturbation, the dominant harmonic has a combination of the fast angles $\psi_{0}, \theta_{0}$. This combination defines an angle with a frequency of order $1 / \sqrt{\nu}$ if $\gamma$ is a quadratic irrational, hence a fast angle but slower than the angles $\psi_{0}$ and $\theta_{0}$. For $\gamma$ with other Diophantine properties the frequency of the fast angle of the dominant harmonic changes but the situation is analogous, see Remarks 5.1 and 7.4 .

\section{A comment on the regularity of the non-autonomous pertur- bation}

Here we consider the unperturbed Hamiltonian (11). A general perturbation $H_{1}(\mathbf{x}, \mathbf{y}, \theta), \theta=$ $\gamma t+\theta_{0}, \gamma \in \mathbb{R} \backslash \mathbb{Q}, \theta_{0} \in[0,2 \pi)$, analytic w.r.t. $\mathbf{x}, \mathbf{y}$ in a compact set $K \subset \mathbb{C}^{2}$ and periodic in $\theta$, will give rise to two sequences of changes in the dominant harmonic of $\Delta F_{1}^{\{1\}}$ and $\Delta F_{2}^{\{1\}}$. Here, we show that if the perturbation $H_{1}(\mathbf{x}, \mathbf{y}, \theta)$ is of class $\mathcal{C}^{p}$ in $\theta$, for some $1 \leq p<\infty$, then the amplitude of the dominant term of the Melnikov function $M\left(\psi_{0}, \theta_{0}\right)$ that measures the splitting between the invariant manifolds $W^{u / s}(\mathbf{0})$ is expected to remain constant as $\nu \rightarrow 0$. On the other hand, if $H_{1}(\mathbf{x}, \mathbf{y}, \theta) \in \mathcal{C}^{\omega}$ then, generically, it is expected to have a decay of the amplitude of the dominant term of $M\left(\psi_{0}, \theta_{0}\right)$ as $\nu \rightarrow 0$. The reason is the following. Consider the Fourier expansion

$$
H_{1}(\mathbf{x}, \mathbf{y}, \theta)=\sum_{k \in \mathbb{Z}} A_{k}(\mathbf{x}, \mathbf{y}) e^{i k \theta} .
$$

We recall that $H_{0}=G_{1}+\nu G_{2}$, and that $G_{1}$ evaluated along the unperturbed separatrix contains a factor periodic in $t$. On the other hand, $H_{1}$ is assumed to be periodic in $\theta$. As explained in Section 5.2, in the exponential part of the Melnikov function there appear terms with

$$
s=j-k \gamma, \quad j, k \in \mathbb{Z}
$$

Assume that $\gamma$ is a Diophantine number and that exist constants $\tilde{c}>0$ and $\tau \geq 1$ such that

$$
|s|>\frac{\tilde{c}}{|k|^{\tau}}
$$

The term whose exponent has the smallest $|s|$ becomes the dominant term of $M\left(\psi_{0}, \theta_{0}\right)$. This term is related to a best approximant of $\gamma$. For the best approximants of $\gamma$ one has $|s|=c|k|^{-\tau}$ for suitable $c>0$ (related to the values $c_{s, m_{1} / m_{2}}$ introduced in Section 17). The exponentially small part of the dominant term of $M\left(\psi_{0}, \theta_{0}\right)$ is expected to be of the form

$$
\exp \left(-\frac{c}{\nu|k|^{\tau}}\right)
$$

We include the effect of the prefactor in front of this exponential part so that we can get more accurate information on the behaviour of the amplitude of $M\left(\psi_{0}, \theta_{0}\right)$. We distinguish two cases according to the regularity of $H_{1}$ w.r.t $\theta$.

1. If $H_{1}(\mathbf{x}, \mathbf{y}, \theta)$ is of class $\mathcal{C}^{p}$ in $\theta$ then the Fourier coefficients decay as

$$
\left|A_{k}(\mathbf{x}, \mathbf{y})\right|=\mathcal{O}\left(|k|^{-p}\right)
$$


The dominant term $d(k)$ of $M\left(\psi_{0}, \theta_{0}\right)$, considering that $\left\{G_{1}, H_{1}\right\}$ has a similar expression to $H_{1}$, is then expected to be of the order

$$
d(k) \approx K \frac{1}{|k|^{p}} \exp \left(-\frac{c}{\nu|k|^{\tau}}\right)
$$

for some $k \in \mathbb{Z}$, where $K \in \mathbb{R}_{+}$is a constant. The maximum of $d(k)$ is attained for $k=k_{M}=(c \tau / \nu p)^{1 / \tau}$ which implies that

$$
d\left(k_{M}\right) \approx C \nu^{p / \tau}, \quad \text { where } \quad C=k(p / c e \tau)^{p / \tau} .
$$

As a conclusion, the dominant term behaves as a power law with respect to $\nu$ and the exponential part of the amplitude remains constant.

2. If, on the other hand, $H_{1}(\mathbf{x}, \mathbf{y}, \theta)$ is of class $\mathcal{C}^{\omega}$ in $\theta$ then the Fourier coefficients decay as

$$
\left|A_{k}(\mathbf{x}, \mathbf{y})\right|=\mathcal{O}\left(e^{-|k| / \rho}\right),
$$

where $\rho>0$ is the distance to the closest singularity of $H_{1}(\mathbf{x}, \mathbf{y}, \theta)$ from the real time axis. Assuming again that $\left\{G_{1}, H_{1}\right\}$ has a similar expression to $H_{1}$, the dominant term of $M\left(\psi_{0}, \theta_{0}\right)$ is expected to be of the order

$$
d(k) \approx K \exp \left(-\frac{|k|}{\rho}\right) \exp \left(-\frac{c}{\nu|k|^{\tau}}\right)
$$

It follows that the maximum of $d(k)$ is attained for $k=k_{M}=(c \tau \rho / \nu)^{1 /(\tau+1)}$ which gives

$$
d\left(k_{M}\right) \approx K \exp \left(-C / \nu^{1 /(\tau+1)}\right), \quad \text { where } \quad C=\frac{\tilde{k}^{1 /(\tau+1)}}{\rho}+\frac{c}{\tilde{k}^{\tau /(\tau+1)}}, \quad \tilde{k}=c \tau \rho .
$$

As a conclusion the dominant term amplitude decreases exponentially. See related comments in Remark 5.1 and Remark [7.4.

\section{Splitting functions, splitting volume and diffusion properties}

As shown in Table 1 for large ranges of $\nu$ the dominant harmonics of both splitting functions coincide. This has some dynamical consequences. Note that this situation can happen in many other systems, and also in situations not necessarily related to the Hamiltonian-Hopf scenario.

In our framework the unperturbed system has a 2-dimensional homoclinic surface given by the invariant manifolds of the origin that coincide $\left(W^{u}=W^{s}\right.$ for the unperturbed system) and which is given by $G_{1}=G_{2}=0$. The invariant manifolds $W^{u}$ and $W^{s}$ split for $\nu>0$. Let $\Delta F_{1}^{\{1\}}$ and $\Delta F_{2}^{\{1\}}$ the splitting functions, measuring the displacement of $W^{u}(0)$ and $W^{s}(0)$ with respect to the unperturbed manifolds. They can be expressed as a Fourier series with combinations of two angles $\psi_{0}, \theta_{0} \in[0,2 \pi)$ of the form $\sin \left(m_{1} \psi_{0}-m_{2} \theta_{0}\right)$. For $\psi_{0}=\theta_{0}=0$ one has a homoclinic point. We look for a basis of $\mathcal{T}_{W^{u}}(0,0)$ (resp. $\left.\mathcal{T}_{W^{s}}(0,0)\right)$, the tangent space to $W^{u}$ (resp. to $W^{s}$ ) at the homoclinic point, and we define the splitting volume at the homoclinic point to be the determinant between the four vectors of the basis (suitably normalized if necessary), see [16].

Assume that, in the fundamental torus $\mathcal{T}_{\Sigma}$ where the splitting between $W^{u}$ and $W^{s}$ is measured, $W^{u}$ is represented as a graph $g_{u}: \mathbb{R}^{2} \rightarrow \mathbb{R}^{4}$, where $g_{u}\left(\psi_{0}, \theta_{0}\right)=\left(\psi_{0}, \theta_{0}, F_{1}^{u}\left(\psi_{0}, \theta_{0}\right), F_{2}^{u}\left(\psi_{0}, \theta_{0}\right)\right)$. 
Similarly, we consider $g_{s}\left(\psi_{0}, \theta_{0}\right)=\left(\psi_{0}, \theta_{0}, F_{1}^{s}\left(\psi_{0}, \theta_{0}\right), F_{2}^{s}\left(\psi_{0}, \theta_{0}\right)\right)$ the graph representation of $W^{s}$ in the fundamental domain. One has

$$
\mathcal{T}_{W^{u}}(0,0)=\left\langle v_{1}, v_{2}\right\rangle, \quad \mathcal{T}_{W^{s}}(0,0)=\left\langle w_{1}, w_{2}\right\rangle,
$$

where

$$
\begin{aligned}
& v_{1}=\frac{\partial g_{u}}{\partial \psi_{0}}(0,0)=\left.\left(1,0, \frac{\partial F_{1}^{u}}{\partial \psi_{0}}, \frac{\partial F_{2}^{u}}{\partial \psi_{0}}\right)\right|_{(0,0)} ^{\top}, v_{2}=\frac{\partial g_{u}}{\partial \theta_{0}}(0,0)=\left.\left(0,1, \frac{\partial F_{1}^{u}}{\partial \theta_{0}}, \frac{\partial F_{2}^{u}}{\partial \theta_{0}}\right)\right|_{(0,0)} ^{\top}, \\
& w_{1}=\frac{\partial g_{s}}{\partial \psi_{0}}(0,0)=\left.\left(1,0, \frac{\partial F_{1}^{s}}{\partial \psi_{0}}, \frac{\partial F_{2}^{s}}{\partial \psi_{0}}\right)\right|_{(0,0)} ^{\top}, w_{2}=\frac{\partial g_{s}}{\partial \theta_{0}}(0,0)=\left.\left(0,1, \frac{\partial F_{1}^{s}}{\partial \theta_{0}}, \frac{\partial F_{2}^{s}}{\partial \theta_{0}}\right)\right|_{(0,0)} ^{\top} .
\end{aligned}
$$

The splitting volume at the homoclinic point at $\left(\psi_{0}, \theta_{0}\right)=(0,0)$ is defined as

$$
V=\operatorname{det}\left(v_{1}, v_{2}, w_{1}, w_{2}\right)
$$

and one has

$$
V=a_{1} b_{2}-b_{1} a_{2}
$$

where

$$
a_{i}=\frac{\partial F_{i}^{u}}{\partial \psi_{0}}(0,0)-\frac{\partial F_{i}^{s}}{\partial \psi_{0}}(0,0), \quad b_{i}=\frac{\partial F_{i}^{u}}{\partial \theta_{0}}(0,0)-\frac{\partial F_{i}^{s}}{\partial \theta_{0}}(0,0), \quad i=1,2 .
$$

The volume $V$ is a quantity related to local diffusive properties: if $V>0$ then the system generically shows some diffusion. If $V=0$ there is no possibility of having "first order" diffusion (nothing prevents on having a much slower diffusion process if the manifolds have non-transversal intersection or intersect transversally at some other homoclinic).

Note that if $\Delta F_{1}^{\{1\}}\left(\psi_{0}, \theta_{0}\right)=c \Delta F_{2}^{\{1\}}\left(\psi_{0}, \theta_{0}\right)$ for some $c \in \mathbb{R} \backslash\{0\},\left(\psi_{0}, \theta_{0}\right) \in \mathcal{T}_{\Sigma}$, then $a_{1}=c a_{2}$ and $b_{1}=c b_{2}$, and $V=0$. For example, as an illustration, if one has

$$
\Delta F_{1}^{\{1\}}=A \sin \left(m_{1} \psi_{0}-m_{2} \theta_{0}\right), \text { and } \Delta F_{2}^{\{1\}}=B \sin \left(m_{1} \psi_{0}-m_{2} \theta_{0}\right),
$$

for $m_{1}, m_{2}$ related to an approximant of $\gamma=(\sqrt{5}-1) / 2$ then the splitting volume is 0 , and no "first order" diffusion is expected. The behaviour of $V$ as a function of $\nu$ is displayed in Fig. 17. We observe on the right plot that for $\nu=\nu_{1}$, where $\log _{2} \nu_{1} \approx-8.391$, it seems $V=0$. The same happens for $\nu=\nu_{3}, \log _{2} \nu_{3} \approx-11.202$. In fact, for $\nu_{1}$ and $\nu_{3}$ there is a change of sign of the determinant $V$. These values of $\nu$ are close to local maxima of the values of $\Delta F_{i}^{\{1\}}$, see Figs. 6 and 5. For values of $\nu$ in an interval around $\nu_{1}$ (respectively, around $\nu_{3}$ ) the dominant harmonic for both $\Delta F_{1}^{\{1\}}$ and $\Delta F_{2}^{\{1\}}$ corresponds to $\left(m_{1}, m_{2}\right)=(5,8)$ (respectively, to $\left.\left(m_{1}, m_{2}\right)=(13,21)\right)$. The nearby harmonics are much smaller and, hence, the splitting functions $\Delta F_{i}^{\{1\}}$ are close to be proportional. Between $\nu_{1}$ and $\nu_{3}$ there is a range of $\nu$ values for which the dominant harmonic is $\left(m_{1}, m_{2}\right)=(8,13)$. This harmonic has the maximum contribution to $\Delta F_{i}^{\{1\}}$ for $\nu=\nu_{2}, \log _{2}\left(\nu_{2}\right) \approx-9.85$, where $V$ has a minimum, but the signs of the dominant harmonic and the nearby ones play a role and $V \neq 0$. We postpone further investigations on the relation between the behaviour of $V$ and the relative position of the invariant manifolds in different ranges of the small parameter for future works.

\section{E On the visible and hidden harmonics of the splitting function related to best approximants}

In Section 7.6 we have shown examples where the approximant associated to one of the best approximants of $\gamma$ is hidden. By hidden harmonic we refer to an harmonic related to a best 

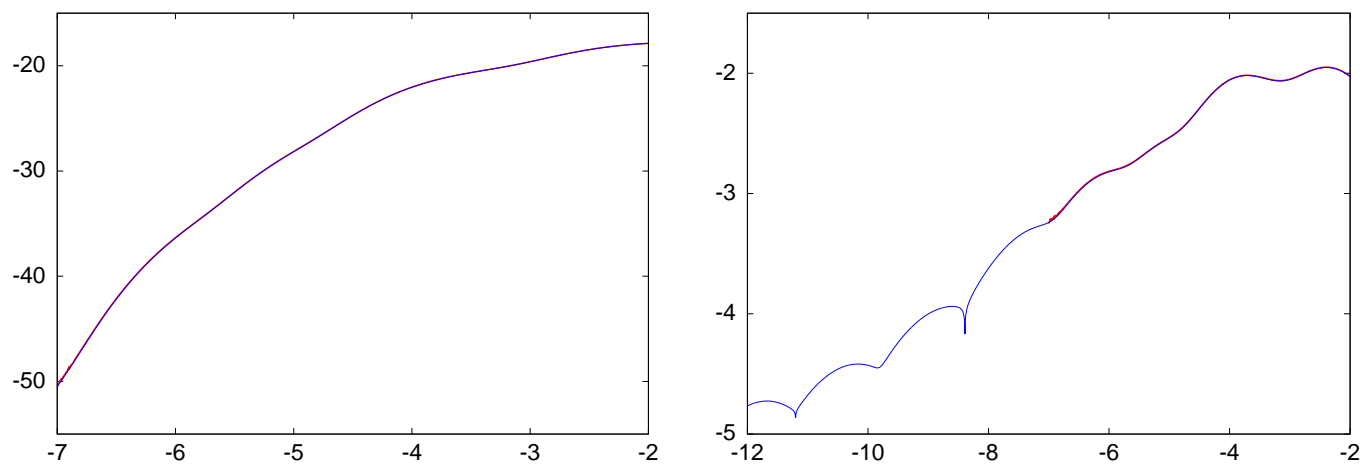

Figure 17: We consider $\epsilon=10^{-3}$ and $\gamma=(\sqrt{5}-1) / 2$. Left: We display $\log (|V|)$ as a function of $\log _{2}(\nu)$. The red points display $V$ computed from a direct computation via a numerical computation of the invariant manifolds. The blue points is an estimate of $V$ based on the dominant terms of the Poincaré-Melnikov approximation. The blue points almost coincide with the red ones and, hence, red points are almost hidden. Right: We plot $\log \left(|V| / \epsilon^{2}\right) \sqrt{\nu}$ for $\nu<2^{-7}$, where $V$ is estimated from the dominant terms Poincaré-Melnikov approximation.

approximant which never becomes dominant: for all values of $\nu$ there is (are) other harmonic(s) which give a larger contribution to the splitting function. Nevertheless we do not have found examples where two (or several) successive harmonics related to best approximants of $\gamma$ are hidden.

In this appendix we explain why some best approximants give a smaller contribution to the splitting, but they are dominant in some narrow range (see Fig. 12 for the case $\gamma=e-2$ and, similarly, the top right plot in Fig. 14 for other $\gamma$ ) while others are not dominant (as seen in the bottom left plot in Fig. 14). We emphasize that this is not related to the concrete Diophantine properties of $\gamma$ but to the different behaviour of the error of the approximations which, as explained in Section 7.6.1, is due to the successive quotients in the CFE of $\gamma$.

Of course, it is easy to produce examples in which several successive best approximants are not dominant. Simply consider that the harmonics related to these approximants have an extremely small amplitude. Here we will consider the case when the amplitudes decrease uniformly in an exponential way and we will show that it is not possible to have two consecutive non-dominant best approximants if some nearby quotients are large. See [9] for more general cases.

Assume we have a perturbation depending on a spatial component $x$ and on time and that the variable $x$ rotates with constant angular velocity, say 1 , while the temporal part has frequency $\gamma$. We also assume that the amplitudes of the harmonics, due to $x$ and to $t$ decrease exactly in an exponential way. Let $N_{n} / D_{n}$ be a best approximant to $\gamma$ and assume, similarly to what was presented in Remark 5.1] in Section 5.2, that the contribution to the Poincaré-Melnikov integral is of the form

$$
\epsilon A \nu^{B} \exp \left(-N_{n} \rho_{1}-D_{n} \rho_{2}\right) \exp \left(\frac{-C s}{\nu}\right),
$$

where $s=\left|N_{n}-\gamma D_{n}\right|$ and that in the problem at hand $C=\pi / 2$. As we are interested in what happens for fixed $\epsilon$ and a given value of $\nu$ the factor $\epsilon A \nu^{B}$ is irrelevant. As shown in Section 7.6.1, $s$ can be expressed as $\left(N_{n} c_{s, n}\right)^{-1}$, where $c_{s, n}$ is given in (40).

To find the maximal contribution is equivalent to find the minimum of minus the exponent. 
Furthermore one has $D_{n}=\left(N_{n} \mp s\right) / \gamma$ where the sign $\mp$ depends on the sign of $s$. Hence

$$
N_{n} \rho_{1}+D_{n} \rho_{2}=N_{n}\left(\rho_{1}+\rho_{2} / \gamma\right)\left(1+\mathcal{O}\left(N_{n}^{-2}\right)\right)
$$

and we also recall that

$$
c_{s, n}=\left(\left(q_{+, n}+1 / q_{-, n}\right) / \gamma\right)\left(1+\mathcal{O}\left(N_{n}^{-2}\right)\right),
$$

where $q_{+, n}$ and $q_{-, n}$ are expressed in terms of quotients of the CFE. Assuming we are dealing with large values of $N_{n}$ the relative contributions of order $N_{n}^{-2}$ will be neglected for simplicity. Furthermore one can introduce $\rho=\rho_{1}+\rho_{2} / \gamma$ to have the following simpler expression for minus the exponent

$$
\rho N_{n}+\frac{C \gamma}{\nu N_{n}\left(q_{+, n}+1 / q_{-, n}\right)} .
$$

Dividing by $\rho$, scaling $\nu$ as $\hat{\nu}=\nu \rho /(C \gamma)$ and denoting $q_{+, n}+1 / q_{-, n}$ by $\hat{c}_{s, n}$, the functions to be studied are of the form

$$
T_{n}(\hat{\nu})=N_{n}+\frac{1}{\hat{\nu} N_{n} \hat{c}_{s, n}} .
$$

In what follows we rename $\hat{\nu}$ and $\hat{c}_{s, n}$ as $\nu$ and $c_{s, n}$, for simplicity. Furthermore, looking at Figs. 5 , 12 and 14, we shall concentrate, as we said, on values of $N_{n}$ which are large (i.e., $\nu$ small) to see, under the current assumptions, which are the best approximants that are not giving the dominant contribution in the splitting. We also note that if $N_{n}<N_{n+1}$ then $T_{n}(\nu)<T_{n+1}(\nu)$ for $\nu$ large and $T_{n}(\nu)>T_{n+1}(\nu)$ for $\nu$ small. This requires to check that the sequence $\left\{1 / N_{n} c_{s, n}\right\}_{n}$ is decreasing. It is an easy check and we refer to [9] for the details. Hence, $T_{n}$ and $T_{n+1}$ coincide only at one point. This is a transversality property.

Assume $\gamma$ is given. We fix our attention to the numerators $N_{n-1}, N_{n}$ and to the quotients $q_{n+j}, j=1,5$. To simplify the notation we shift the subindices by $n$. For completeness we also take into account the value $\alpha_{+}=1 /\left[q_{6} ; q_{7}, \ldots\right]$. We assume that $q_{1}, q_{3}, q_{4}$ are relatively small and that $q_{2}, q_{5}$ very large, say of the order of a large number $Q$, which hereafter will be considered as a parameter. Looking only at the dominant contributions (i.e., neglecting terms of relative size of $\left.\mathcal{O}\left(Q^{-1}\right)\right)$ we have the numerators

$$
N_{1}, \quad N_{2}=q_{2} N_{1}, \quad N_{3}=q_{3} q_{2} N_{1}, \quad N_{4}=q_{2} N_{1}\left(1+q_{4} q_{3}\right) .
$$

The relevant values of $c_{s, j}$ are

$$
c_{s, 1}=q_{2}, \quad c_{s, 2}=q_{3}+1 / q_{4}, \quad c_{s, 3}=q_{4}+1 / q_{3}, \quad c_{s, 4}=q_{5} .
$$

From this we can compute the values of $T_{j}, j=1,4$, skipping terms relatively $\mathcal{O}\left(Q^{-1}\right)$ :

$$
\begin{gathered}
T_{1}(\nu)=N_{1}+\frac{1}{\nu N_{1} q_{2}}, \quad T_{2}(\nu)=q_{2} N_{1}+\frac{q_{4}}{\nu q_{2} N_{1}\left(1+q_{3} q_{4}\right)}, \quad T_{3}(\nu)=q_{3} q_{2} N_{1}+\frac{1}{\nu q_{2} N_{1}\left(1+q_{3} q_{4}\right)}, \\
T_{4}(\nu)=q_{2} N_{1}\left(1+q_{4} q_{3}\right)+\frac{1}{\nu q_{2} N_{1}\left(1+q_{3} q_{4}\right) q_{5}} .
\end{gathered}
$$

Now we look for a value of $\nu$, say $\nu^{*}$, for which one has $T_{1}=T_{4}$. One has $\nu^{*}=\left(N_{1}^{2} q_{2}^{2}\left(1+q_{3} q_{4}\right)\right)^{-1}$ which allows to compute the value $T_{1}\left(\nu^{*}\right)=T_{4}\left(\nu^{*}\right)$. But, as done in the different figures, it is suitable to multiply $T_{i}$ by $\sqrt{\nu^{*}}$. In this way one obtains plots similar to the ones in the figures, just changing the sign. Let us denote $\sqrt{\nu^{*}} T_{j}\left(\nu^{*}\right)$ by $\hat{T}_{j}\left(\nu^{*}\right)$ and then

$$
\hat{T}_{1}\left(\nu^{*}\right)=\sqrt{1+q_{3} q_{4}}=\hat{T}_{4}\left(\nu^{*}\right) .
$$


At that value of $\nu^{*}$ there is a change: $T_{1}<T_{4}$ for $\nu>\nu^{*}$ while $T_{1}>T_{4}$ otherwise. But, what are $T_{2}$ and $T_{3}$ doing? A simple computation gives

$$
\hat{T}_{2}\left(\nu^{*}\right)=\frac{1+q_{4}}{\sqrt{1+q_{3} q_{4}}}, \quad \hat{T}_{3}\left(\nu^{*}\right)=\frac{1+q_{3}}{\sqrt{1+q_{3} q_{4}}} .
$$

Therefore, if $q_{3}<q_{4}$ one has $\hat{T}_{3}\left(\nu^{*}\right)<\hat{T}_{2}\left(\nu^{*}\right) \leq \hat{T}_{1}\left(\nu^{*}\right)$, and the last inequality becomes an equality if, and only if, $q_{3}=1$. This, together with the transversality property mentioned above, implies that the best approximant associated to $N_{3}$ is dominant. In a similar way, if $q_{4}<q_{3}$ one has $\hat{T}_{2}\left(\nu^{*}\right)<\hat{T}_{3}\left(\nu^{*}\right) \leq \hat{T}_{1}\left(\nu^{*}\right)$ with equality between the last two if, and only if, $q_{4}=1$.

Summarizing, under the current conditions, if either $q_{3}$ or $q_{4}$ are different from 1 , then at least one of them is the dominant term in a range between the dominance of the best approximant associated to $N_{1}$ and the one associated to $N_{4}$.

It remains to investigate what happens if $q_{3}=q_{4}=1$. In that case the approximation neglecting relative terms $\mathcal{O}\left(Q^{-1}\right)$ gives that $T_{1}(\nu), T_{2}(\nu), T_{3}(\nu)$ and $T_{4}(\nu)$ intersect at the same point. Numerical evidence is that $T_{2}(\nu)$ or $T_{3}(\nu)$ or both of them dominate in some narrow range. Which one dominates or in which cases both of them dominate in suitable ranges depends, mainly, on the ratio $q_{2} / q_{5}$. Let us prove the theoretical facts confirming this numerical evidence.

Hence we take $q_{3}=q_{4}=1$ and recompute $N_{j}$ and $c_{s, j}, j=1, \ldots, 4$, taking into account terms whose relative value is $\mathcal{O}\left(Q^{-1}\right)$. We note that $N_{2}=q_{2} N_{1}+N_{0}=N_{1}\left(q_{2}+N_{0} / N_{1}\right)$. For simplicity we denote $N_{0} / N_{1}$ by $\alpha$. One easily obtains

$$
N_{2}=N_{1}\left(q_{2}+\alpha\right), \quad N_{3}=N_{1}\left(q_{2}+\alpha+1\right), \quad N_{4}=N_{1}\left(2 q_{2}+2 \alpha+1\right) .
$$

In a similar way we obtain the following values for $c_{s, j}$,

$$
c_{s, 1}=q_{2}+\frac{1}{2}+\alpha, \quad c_{s, 2}=2-\frac{1}{q_{5}}+\frac{1}{q_{2}}, \quad c_{s, 3}=2-\frac{1}{q_{2}}+\frac{1}{q_{5}}, \quad c_{s, 4}=q_{5}+\alpha_{+}+\frac{1}{2},
$$

where we recall that $\alpha_{+}=1 /\left[q_{6} ; q_{7}, \ldots\right]$.

Let us denote by $\nu_{i, j}$ the value of $\nu$ for which $T_{i}=T_{j}$. One has

$$
\nu_{i, j}=\frac{\left(N_{i} c_{s, i}\right)^{-1}-\left(N_{j} c_{s, j}\right)^{-1}}{N_{j}-N_{i}} .
$$

It is immediate to obtain expressions for $\nu_{i, j}, 1 \leq i<j \leq 4$, but for shortness we display the values obtained after shifting and scaling: $\nu_{i, j}^{*}=\left(\nu_{i, j} N_{1}^{2} q_{2}^{2}-1 / 2\right) q_{2}$. They are

$$
\begin{aligned}
& \nu_{1,2}^{*}=\frac{1}{4}-\frac{q_{2}}{4 q_{5}}-\alpha, \quad \nu_{1,3}^{*}=-\frac{1}{4}+\frac{q_{2}}{4 q_{5}}-\alpha, \quad \nu_{1,4}^{*}=-\frac{1}{4}-\frac{q_{2}}{4 q_{5}}-\alpha, \\
& \nu_{2,3}^{*}=\left(\frac{q_{2}}{2 q_{5}}-\frac{1}{2}\right) q_{2}, \quad \nu_{2,4}^{*}=-\frac{3}{4}-\frac{q_{2}}{4 q_{5}}-\alpha, \quad \nu_{3,4}^{*}=-\frac{1}{4}-\frac{3 q_{2}}{4 q_{5}}-\alpha .
\end{aligned}
$$

We note that to have a more precise value for $\nu_{2,3}^{*}$ it would be necessary to carry out some expansion with relative order $\mathcal{O}\left(Q^{-2}\right)$, specially if $q_{2}$ and $q_{5}$ are close. But it is not necessary for our purposes.

From the previous expressions one has

$$
\max \left\{\nu_{2,4}^{*}, \nu_{3,4}^{*}\right\}<\nu_{1,4}^{*}<\min \left\{\nu_{1,2}^{*}, \nu_{1,3}^{*}\right\},
$$


showing that when $\nu$ decreases, either $T_{2}(\nu)$ or $T_{3}(\nu)$ intersect $T_{1}(\nu)$ before the intersection of $T_{1}(\nu)$ and $T_{4}(\nu)$ and, on the other side, either $T_{2}(\nu)$ or $T_{3}(\nu)$ intersect $T_{4}(\nu)$ after the intersection of $T_{1}(\nu)$ and $T_{4}(\nu)$.

Hence, either the harmonic associated to $N_{2}$ or the one associated to $N_{3}$ (or both of them in some small ranges) dominate the splitting between the dominances of the harmonics associated to $N_{1}$ and to $N_{4}$. The numerical evidence in many examples is that both of them can be seen only if $\left|q_{2}-q_{5}\right| \leq 1$.

If $q_{2}<q_{5}$ but they are not so close, then $\nu_{1,2}^{*}>\nu_{1,3}^{*}$ and $\nu_{2,4}^{*}<\nu_{3,4}^{*}$, showing that between the dominances related to $N_{1}$ and to $N_{4}$ there is a range of dominance of $N_{2}$. The roles of $N_{2}$ and $N_{3}$ are exchanged if $q_{5}<q_{2}$ but they are not so close.

Remark E.1. We have considered some transcendental frequencies like $\pi$ and $\exp (\sqrt{5}-1 / 2)$. From the $10^{7}$ first quotients of the CFE we observe that it is reasonable to accept them as "typical" irrational numbers: the geometric mean of the quotients of the CFE tends to the Khinchin constant (see, e.g., https://oeis.org/A002210 for numerical values) and the ratio of increase of the denominators, measured as $\lim _{n \rightarrow \infty} \log \left(D_{n}\right) / n$ tends to the Lévy's constant $\pi^{2} /(12 \log (2))$. In all the cases the hidden harmonics that have been detected (around $27.9 \%$ of the total number of approximants) are isolated. Further details can be found in 9 .

\section{Acknowledgments}

This work has been supported by grants MTM2016-80117-P (Spain) and 2017-SGR-1374 (Catalonia). We also thank the MINECO grant MDM-2014-0445 (Spain). We are specially indebted to A. Delshams, M. Gonchenko and V. Gelfreich for several discussions on related topics. We also thank J. Timoneda for maintaining the computing facilities of the Dynamical Systems Group of the Universitat de Barcelona, that have been largely used in this work.

\section{References}

[1] A. Delshams, V. Gelfreich, A. Jorba, and T.M. Seara. Exponentially small splitting of separatrices under fast quasiperiodic forcing. Comm. Math. Phys., 189(1):35-71, 1997.

[2] A. Delshams, M. Gonchenko, and P. Gutiérrez. Continuation of the exponentially small transversality for the splitting of separatrices to a whiskered torus with silver ratio. Regul. Chaotic Dyn., 19(6):663-680, 2014.

[3] A. Delshams, M. Gonchenko, and P. Gutiérrez. Exponentially small asymptotic estimates for the splitting of separatrices to whiskered tori with quadratic and cubic frequencies. Electron. Res. Announc. Math. Sci., 21:41-61, 2014.

[4] A. Delshams, M. Gonchenko, and P. Gutiérrez. Exponentially small lower bounds for the splitting of separatrices to whiskered tori with frequencies of constant type. Internat. J. Bifur. Chaos Appl. Sci. Engrg., 24(8):1440011, 12, 2014.

[5] A. Delshams and P. Gutiérrez. Exponentially Small Splitting of Separatrices for Whiskered Tori in Hamiltonian Systems. Journal of Mathematical Sciences, 128(2):2726 -2745, 2005.

[6] A. Delshams and R. Ramírez-Ros. Singular separatrix splitting and Melnikov method: An experimental study. Experiment. Math., 8(1):29-48, 1999. 
[7] J.C. Van der Meer. Nonsemisimple 1: 1 resonance at an equilibrium. Celestial Mech., 27(2):131-149, 1982.

[8] C. Elphick, E. Tirapegui, M.E. Brachet, P. Coullet, and G. Iooss. A simple global characterization for normal forms of singular vector fields. Physica D, 29(1-2):95-127, 1987.

[9] E. Fontich, C. Simó, and A. Vieiro. On the "hidden" harmonics associated to best approximants due to quasi-periodicity, 2018. Preprint.

[10] J.P. Gaivão and V. Gelfreich. Splitting of separatrices for the Hamiltonian-Hopf bifurcation with the Swift-Hohenberg equation as an example. Nonlinearity, 24(3):677-698, 2011.

[11] V.G. Gelfreich and V.F. Lazutkin. Splitting of separatrices: perturbation theory and exponential smallness. Russian Math. Surveys, 56(3):499-558, 2001.

[12] M. Guardia and T.M. Seara. Exponentially and non-exponentially small splitting of separatrices for the pendulum with a fast meromorphic perturbation. Nonlinearity, 24(5):1367$1412,2012$.

[13] H. Hanßmann. Local and semi-local bifurcations in Hamiltonian dynamical systems: Results and examples. Lecture Notes in Mathematics, 1893. Springer-Verlag, 2007.

[14] A.Ya. Khinchin. Continued Fractions. The University of Chicago Press, Chicago, Ill.London, 1964.

[15] L.M. Lerman and Ya.L. Umanskiu. Classification of four-dimensional integrable hamiltonian systems and poisson actions of $\mathbb{R}^{2}$ in extended neighborhoods of simple singular points. I. Russian Acad. Sci. Sb. Math., 77:511-542, 1994.

[16] P. Lochak, J.-P. Marco, and D. Sauzin. On the splitting of invariant manifolds in multidimensional near-integrable Hamiltonian systems. Mem. Amer. Math. Soc., 163(775), 2003.

[17] P.D. McSwiggen and K.R. Meyer. The evolution of invariant manifolds in Hamiltonian-Hopf bifurcations. J. Differential Equations, 189(2):538-555, 2003.

[18] K.R. Meyer and G. Hall. Introduction to Hamiltonian dynamical systems and the N-body problem. Applied Mathematical Sciences, 90. Springer-Verlag, New York, 1992.

[19] K.R. Meyer and D.S. Schmidt. Periodic orbits near $\mathcal{L}_{4}$ for mass ratios near the critical mass ratio of Routh. Celestial Mech., 4:99-109, 1971.

[20] A. Neishtadt. The separation of motions in systems with rapidly rotating phase. Prikladnaja Matematika i Mekhanika, 48:133-139, 1984.

[21] J. Palacián and P. Yanguas. Reduction of polynomial Hamiltonians by the construction of formal integrals. Nonlinearity, 13(4):1021-1054, 2000.

[22] C. Robinson. Melnikov method for autonomous Hamiltonians. In Donald G. Saari and Zhihong Xia, editors, Hamiltonian dynamics and celestial mechanics, Contemporary Mathematics, pages 45-53. American Mathematical Society, Providence, RI, 1996.

[23] D.A. Salamon. The Kolmogorov-Arnold-Moser theorem. Math. Phys. Electron. J., 10:3-37, 2004 .

[24] J.A. Sanders. Melnikov's method and averaging. Cel. Mech., 28:171-181, 1982. 
[25] C. Simó. Averaging under Fast Quasiperiodic Forcing. In J. Seimenis, editor, Hamiltonian Mechanics: Integrability and Chaotic Behaviour, volume 331 of NATO Adv. Sci. Inst. Ser. B Phys., pages 13-34, Toruń, Polland, 1994. Plenum Press, New York.

[26] C. Simó and C. Valls. A formal approximation of the splitting of separatrices in the classical Arnold's example of diffusion with two equal parameters. Nonlinearity, 14:1707-1760, 2001.

[27] A.G. Sokolskil. On the stability of an autonomous Hamiltonian system with two degrees of freedom in the case of equal frequencies. J. Appl. Math. Mech., 38:741-749, 1974. Translated from Prikl. Mat. Meh. 38, 791-799 (Russian), 1974.

[28] J. Williamson. On the normal forms of linear canonical transformations in dynamics. American Journal of Mathematics, 59(3):599-617, 1937. 